\title{
Rational design of allosteric nanodevices based on DNA triple helix
}

\section{Supporting Information}

Tianqing Zhang ${ }^{*}$, Bryan Wei*

School of Life Sciences, Tsinghua University-Peking University Center for Life Sciences, Center for Synthetic and Systems Biology, Tsinghua University, Beijing 100084, China

* Correspondence and requests for materials should be addressed to Bryan Wei (email: bw@tsinghua.edu.cn); Tianqing Zhang (email: ztq17@mails.tsinghua.edu.cn); 


\section{Materials and Methods}

Oligonucleotides. DNA sequence of Z-motif was adapted and optimized from Mao group. All optimized DNA sequences were tested in NUPACK to prevent self-sequence complementation. All non-labeled DNA strands were purchased from Sangon Biotech (Shanghai) Co. Ltd (with PAGE purification). Fluorophore labeled DNA strands were purchased from Sangon Biotech (Shanghai) Co. Ltd (with HPLC purification). Details about DNA sequences were showed in Sequences part in Supplementary Information.

Chemicals. Reagent-grade chemicals (Tris base, Acetic acid, Magnesium chloride, EDTA, Boric acid, Nickel chloride, SYBR safe, Thiazole Orange) commercially available were used without further purifications.

\section{Buffers.}

1. DNA nanodevice forming buffer:

Buffer 1(10×): $400 \mathrm{mM}$ tris base, $200 \mathrm{mM}$ acetic acid, $10 \mathrm{mM}$ EDTA and $125 \mathrm{mM}$ magnesium chloride, $\mathrm{pH}$ is adjusted to $\sim 8$;

2. AFM scanning buffer:

Buffer 2: $20 \mathrm{mM}$ tris base, $0.5 \mathrm{mM}$ EDTA, $10 \mathrm{mM}$ magnesium chloride, $\mathrm{pH}$ is adjusted to $\sim 8$.

Preparation of DNA nanodevices. For all DNA nanodevices, final concentration of each DNA strand in $40 \mu \mathrm{L}$ structure forming buffer (Buffer 1 to $1 \times$ ) was $200 \mathrm{nM}$. The DNA mixture solutions were then thermally annealed in 2 hours $\left(95^{\circ} \mathrm{C}\right.$ for $5 \mathrm{~min}, 65^{\circ} \mathrm{C}$ for $30 \mathrm{~min}, 50{ }^{\circ} \mathrm{C}$ for $30 \mathrm{~min}, 37^{\circ} \mathrm{C}$ for $30 \mathrm{~min}$, and $22{ }^{\circ} \mathrm{C}$ for $30 \mathrm{~min}$ ). For Boolean operations, add all pre-annealed inputs into the annealed DNA mixture solution with their stoichiometric molar ratio (1:1 in logic gates operation and 2:1 in Majority operations) and stand overnight at $22{ }^{\circ} \mathrm{C}$ to give final $50 \mathrm{uL} 160 \mathrm{nM}$ motif solution.

Energetics simulation. All allosteric DNA nanodevices was engineered as branchstem-branch structures in which one or two active sites are partially blocked to varying 
degrees by hydrogen bonds. Such blocked structures are in thermodynamic equilibrium with unblocked state in which the hydrogen bonds in active sites are dissolved. When TFO recognizes the triplex-forming region embedded in the bulge region, initiating triplex-induced strand displacement, the thermodynamic equilibrium shifts towards this latter unblocked conformation. For each of the allosteric nanodevices, the predicted standard free energies of those two switching states were determined in silico using NUPACK Utilities module, setting $\mathrm{T}=22{ }^{\circ} \mathrm{C},\left[\mathrm{Na}^{+}\right]=0.05 \mathrm{M},\left[\mathrm{Mg}^{2+}\right]=12.5 \mathrm{mM}(\mathrm{As}$ $\left[\mathrm{Na}^{+}\right]$cannot be set as 0 in NUPACK Utilities module, we choose the minimum value in the allowed range that will be considered as negligible contributions). Based on these values, it was probable to calculate the predicted intrinsic switching equilibrium constant (Ks) of each allosteric DNA nanodevice using the standard thermodynamic equation $\Delta \mathrm{G}=-\mathrm{RT} \cdot \ln (\mathrm{K})$.

Native PAGE. Prepare $12 \%$ native PAGE $\left(1 \times \mathrm{TBE}-\mathrm{Mg}^{2+}\right.$, acrylamide: bis-acrylamide $=19: 1$ ), the running buffer is $1 \times \mathrm{TBE}-\mathrm{Mg}^{2+}$. Gels were run at $120 \mathrm{~V}$ (constant voltage) for $60 \mathrm{~min}$. After electrophoresis, gel was stained by 1x SYBR Safe (Thermo Fisher Scientific Inc.) and scanned in Typhoon FLA 9500 Fluorescent Image Analyzer Scanner (GE Healthcare) at designative channel.

AFM imaging. Add $50 \mu \mathrm{L}$ scanning buffer into freshly cleaved mica, then load $2 \mu \mathrm{L}$ sample and stay for $5 \mathrm{~min}$, then wash 3 times with scanning buffer to remove excess DNA in solution and reload $50 \mu \mathrm{L}$ scanning buffer and $2 \mu \mathrm{L}$ nickel chloride $(10 \mathrm{mM})$ for 2min. AFM images were obtained using a Bruker Multimode8 with Nanoscope V controller (Bruker Corp.). Samples were imaged under liquid ScanAsyst mode with Ctype triangular tips (resonant frequency, $\mathrm{f}_{0}=40-75 \mathrm{kHz}$; spring constant, $\mathrm{k}=0.24 \mathrm{~N} \mathrm{~m}^{-}$ $\left.{ }^{1}\right)$ from the SNL-10 silicon nitride cantilever chip (Bruker Corp.). AFM images were quantified using software ImageJ (Plugins $\rightarrow$ Analyze $\rightarrow$ Cell counter), details can be found in Supplementary Note. 1.

Fluorescence measurements. Fluorescence measurements for Triplex-Thiazole 
Orange (TO) binding were performed in the following protocol: TO-DNA complexes were prepared by incubation of annealed DNA nanodevices with an amount of TO with their stoichiometric molar ratio $(1: 100)$ for 1 hour at $22{ }^{\circ} \mathrm{C}$. Transfer the solution into 3 $\mathrm{K}$ Amicon Ultra centrifugal filter, add $450 \mu \mathrm{L} 1 \times \mathrm{TAE}-\mathrm{Mg}^{2+}$ buffer then centrifuge at $13,000 \times \mathrm{g}$ for $8 \mathrm{~min}$ and discard the centrifugate, repeat for 4 times excess TO and those loosely bound to DNA duplex regions. Transfer residual solution into a $1.5 \mathrm{~mL}$ Eppendorf tube and dilute into constant volume of $100 \mu \mathrm{L}$ with $1 \times \mathrm{TAE}-\mathrm{Mg}^{2+}$. Fluorescence intensity was recorded with Fluorolog ${ }^{\circledR}-3$ spectrofluorometer system (HORIBA, Ltd) using an accessary xenon lamp as the light source. The intensity was recorded at excitation and emission wavelengths of 514 and $555 \mathrm{~nm}$, respectively. Set the excitation and emission slit-widths to $5 \mathrm{~nm}$.

Statistical analysis. The transition ratios of 1 site transition screening and 2 sites transition screening were collected from $\sim 0.1 \mu \mathrm{m}^{2}$ area of AFM images and given by the equation:

(1) $\varphi=\frac{\left[\text { Switch }_{\text {after transition }}\right]}{\left[\text { Switch }_{\text {after transition }]+\left[\text { Switch }_{\text {before transition }}\right]}\right.}$

where $\left[\right.$ Switch $\left.{ }_{\text {after transition }}\right]$ is the number of Z-switches that underwent the

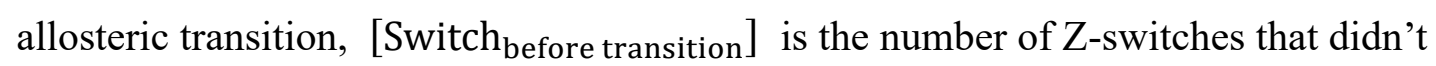
undergo the allosteric transition. To simplify the allosteric transition process, we have divided the whole process into 3 steps (step 1: $\rightarrow$; step 2: $\rightarrow c$; step 3: $\rightarrow$ $\rightarrow$; Supplementary Fig. 3 and Supplementary Fig. 17). We envisioned the binding ratio of TFO with the one strand in Z-switch via Watson-Crick interaction in step 1 is $100 \%$ but the formation and propagation of triplex in step 2 is not $100 \%$. And in step 3, we envisioned that once the Z-switches are allosterically changed by TFO modulator, most of them can assembly into the ladder array with negligible switches

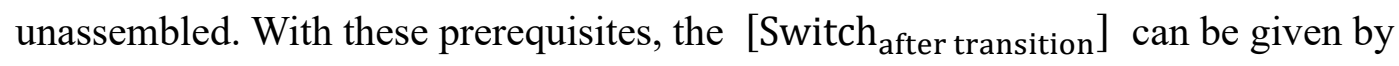

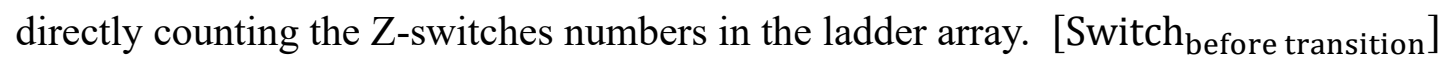
can be considered as the total number of the unassembled Z- switches and those in the comb array (failed transition). Thus,

(2) $\left[\right.$ Switch $\left.{ }_{\text {after transition }}\right]=\left[\right.$ Switch $\left._{\text {ladder array }}\right]$ 
(3) $\left[\right.$ Switch $\left._{\text {before transition }}\right]=\left[\right.$ Switch $\left._{\text {unassembled }}\right]+\left[\right.$ Switch $\left._{\text {comb array }}\right]$

For example, below is the zoom-in AFM images of group D9 screening.
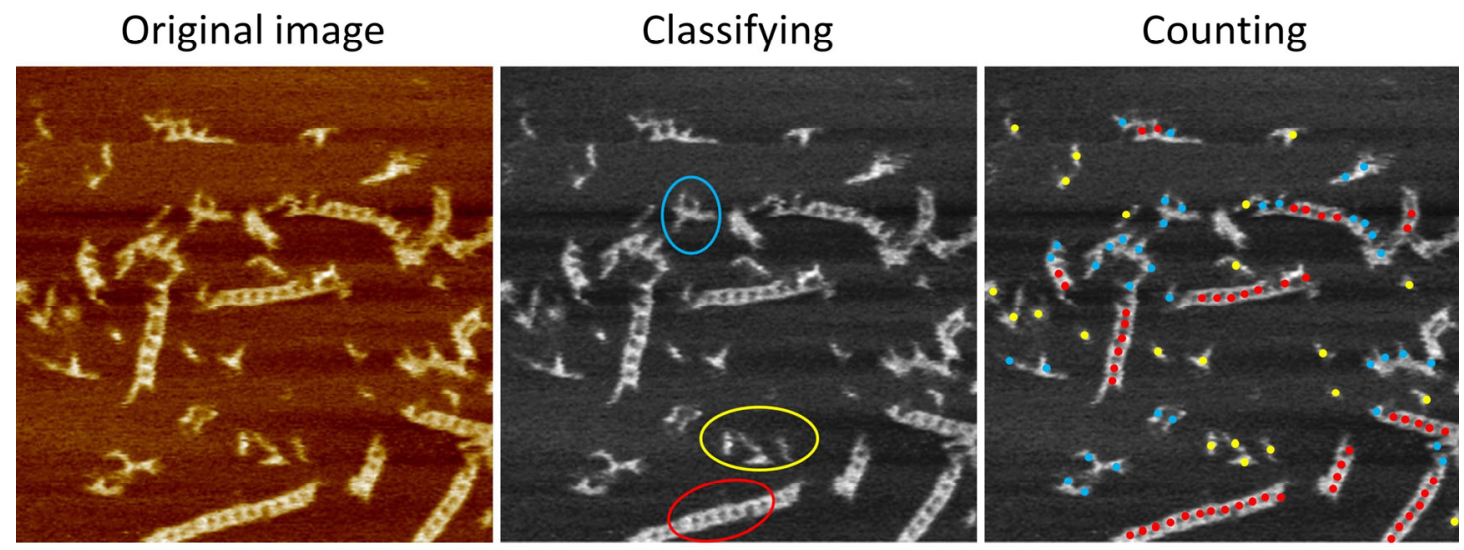

Three kinds of colors represent three different readouts: red color represent the Zswitches in the ladder array; blue color represent the Z-switches in the comb array; yellow color represent the unassembled Z-switches. After classifying and counting these three readouts, we got the transition ratio by combining equation (1)-(3).

To get the transition ratios of logic gates and MAJORITY function experiments, the data were collected from $\sim 1 \mu \mathrm{m}^{2}$ area of AFM images and given by the equation, the statistical method is almost the same apart from some variations in equation (3). In equation (3), the $\left[\right.$ Switch $_{\text {unassembled }}$ is not equal to the yellow readouts from the AFM images. For example, in the NOR gate experiment with input $=[1,0]$ or input $=$ $[0,1]$, the yellow readouts is the total value of $\left[\right.$ Switch $\left._{\text {unassembled }}\right]$ and [Waste]. While it's difficult to confirm the concrete values of the waste on mica surface, thus for the ease of comparison, we defined in these situations that each kind of [Waste]

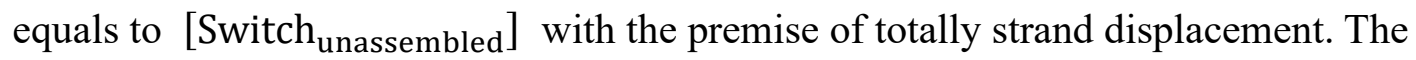
other cases can be handled with the same methodology. 


\section{Supporting Figures}

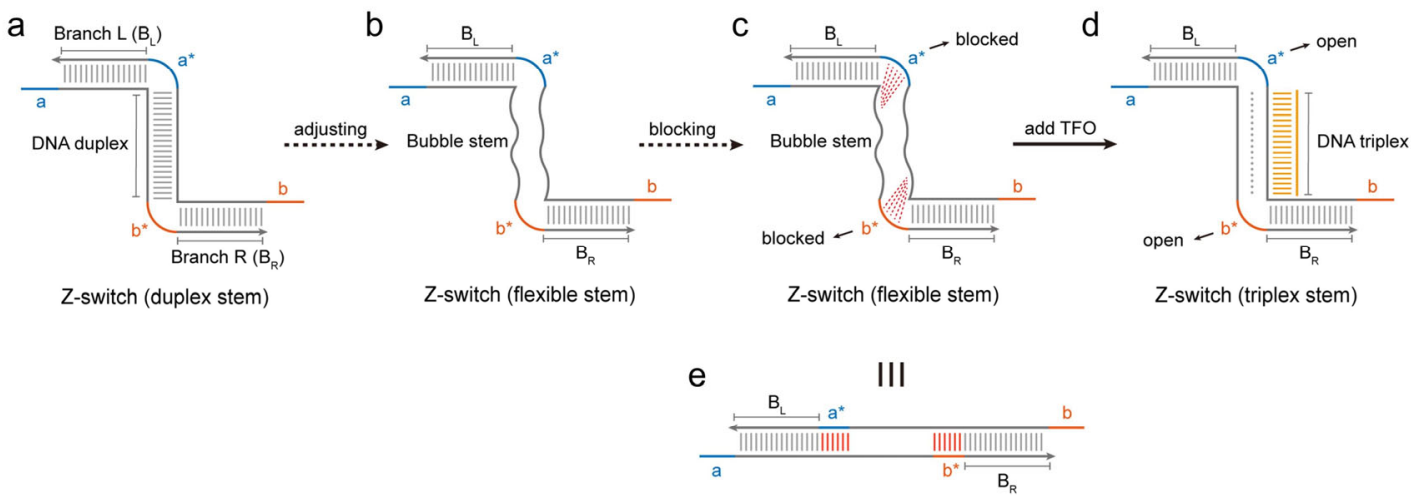

Figure S1. Design of the Z-switch with a triple-helical stem. a, Normal Z-switch with a double-helical stem. Blue and orange arcs (labeled as $a^{*}$ and $b^{*}$, respectively): active sites that can interact with $5^{\prime}$ overhangs of the same color (labeled as a and $b$, respectively). $\mathbf{b}$, Replacing the duplex region of Z-switch with sequence that can form triplex upon TFO binding. c, Blocking the active site $\mathrm{a}^{*}$ and $\mathrm{b}^{*}$ in the Z-switch by complementary base pairing. $\mathbf{d}$, Z-switch with blocked site can be revealed upon TFO binding. 
a

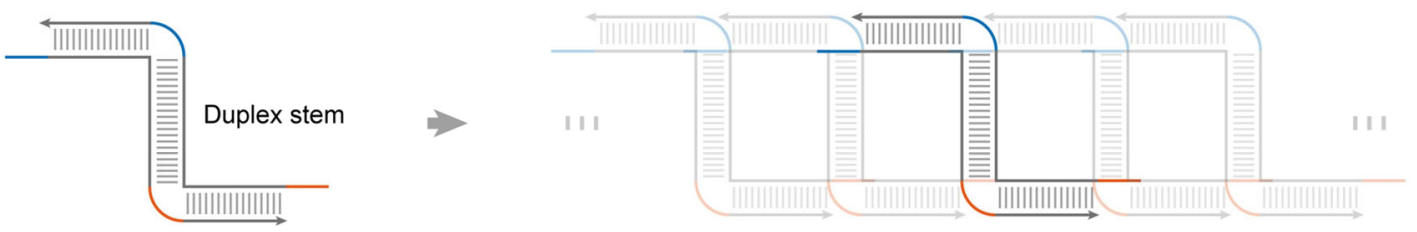

Assembly

b
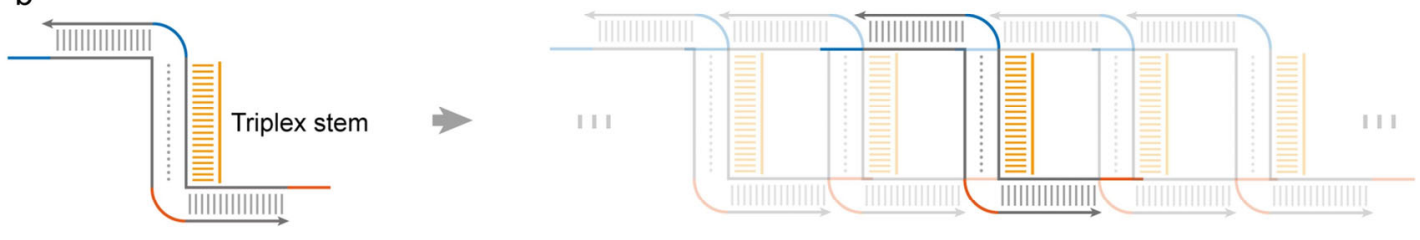

Assembly

Duplex stem

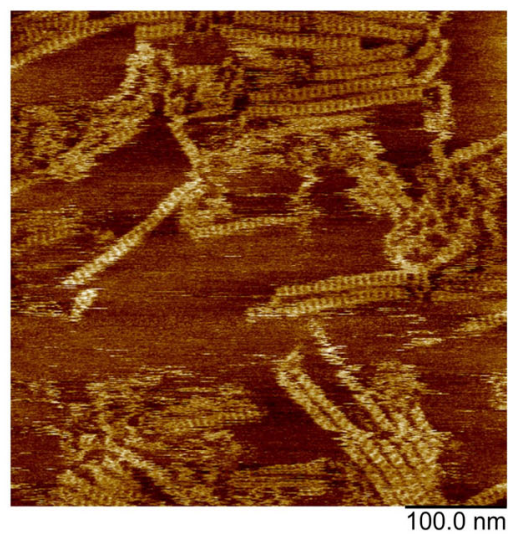

Triplex stem

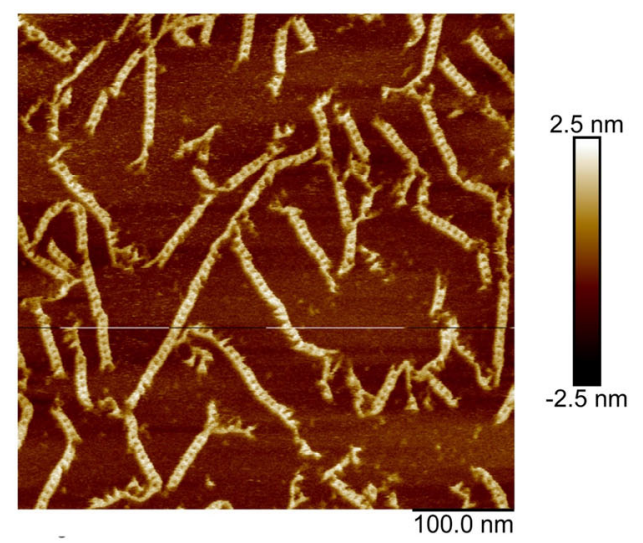

Figure S2. Without blocking their active sites, Z-switches with (a) double-helical stem and (b) triple-helical stem can all assemble into ladder array as observed in the AFM results (bottom panel). 
$d$

a

\begin{tabular}{|c|c|}
\hline Blocked & \\
\hline|||||||||||||||||||||| & |||||||||||||||||||||||| \\
\hline
\end{tabular}

b

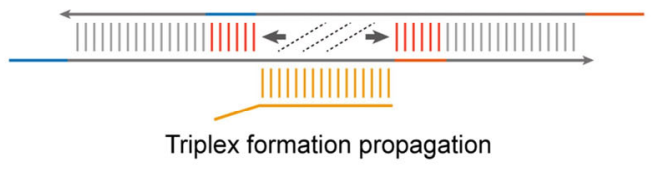

Figure S3. Allosteric transition of triplex-induced Z-switches with $\mathbf{2}$ sites blocked. a, The blocked state of Z-switch. b, Triplex formation and propagation. c, Triplex propagation induces strand displacement and reopens the blocked site. d, The ON state after triplex-induced conformational change. 


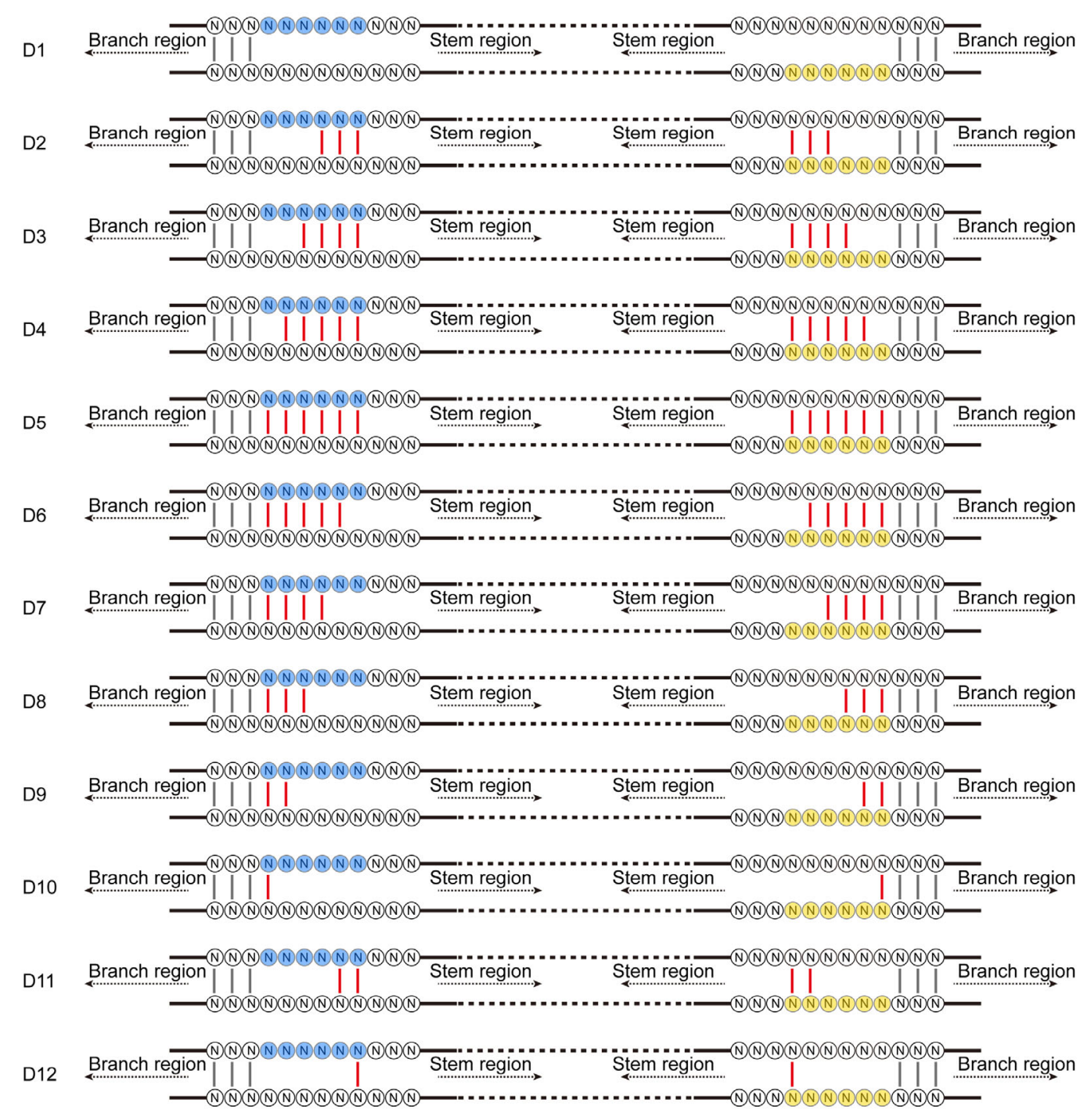

Figure S4. Schematic showing the allosteric Z-switches with 12 different levels of blocking on both active sites ( site $\mathrm{a}^{*}$ and $\mathrm{b}^{*}$ ). Blue region represents active site $\mathrm{a}^{*}$ and yellow region represents active site $b^{*} .5$ ' overhangs are not displayed. All nucleic acids are marked as $\mathbf{N}$ and vertical lines represent the hydrogen bond. Red vertical lines represent the blocking on active site $\mathrm{a}^{*}$ and $\mathrm{b}^{*}$. 
2 sites allosteric transition screening
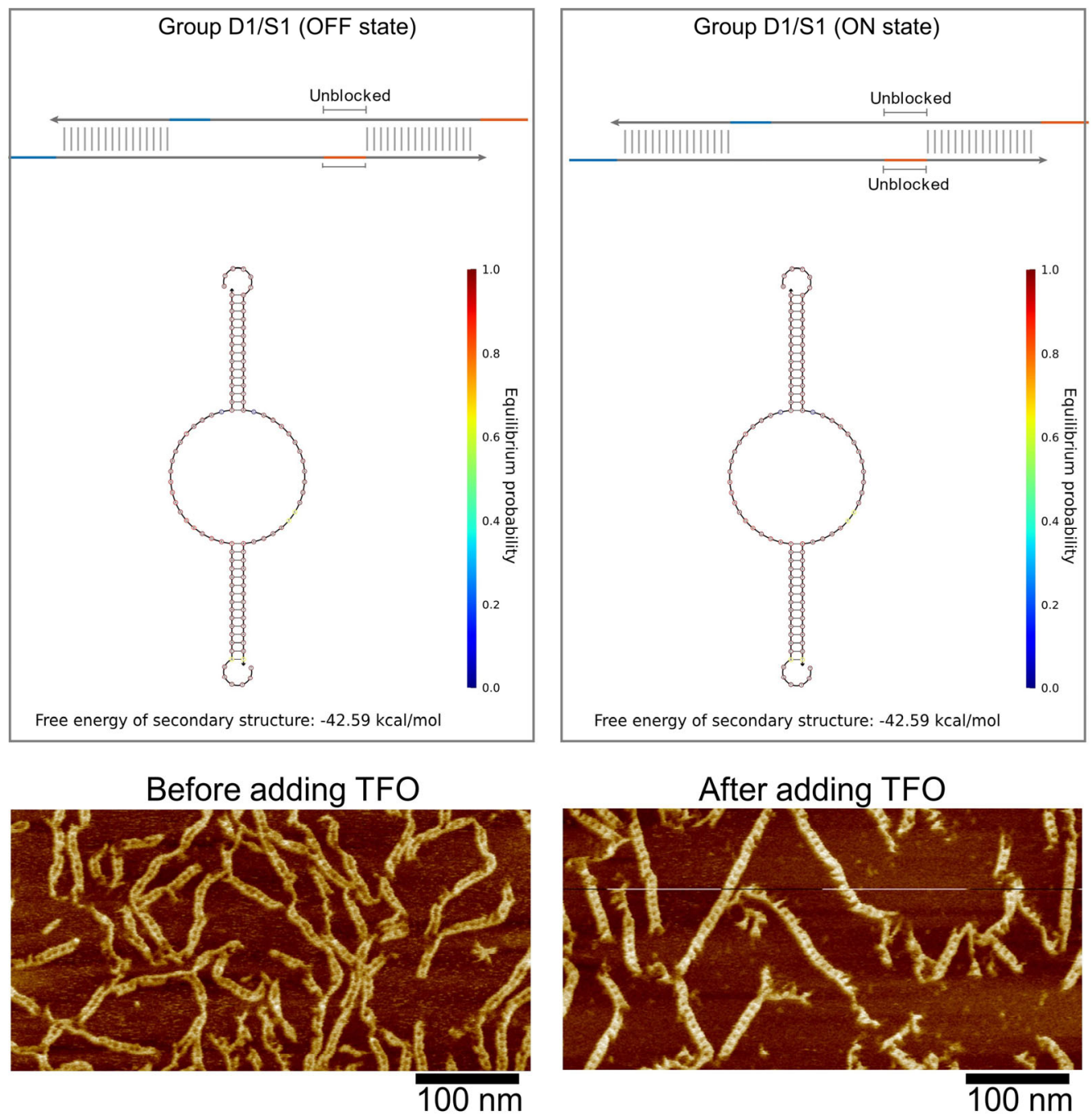

Figure S5. Schematic and simulated energetics parameter of the OFF state (left) and ON state (right) group D1 for two sites allosteric transition screening (top panel). AFM images of group D1 before (left) and after (right) adding TFO modulator (bottom panel). Scale bars, $100 \mathrm{~nm}$.

Note that the design and sequence of D1 variant of two sites screening are the same as those of S1 variant of single site screening. 
2 sites allosteric transition screening
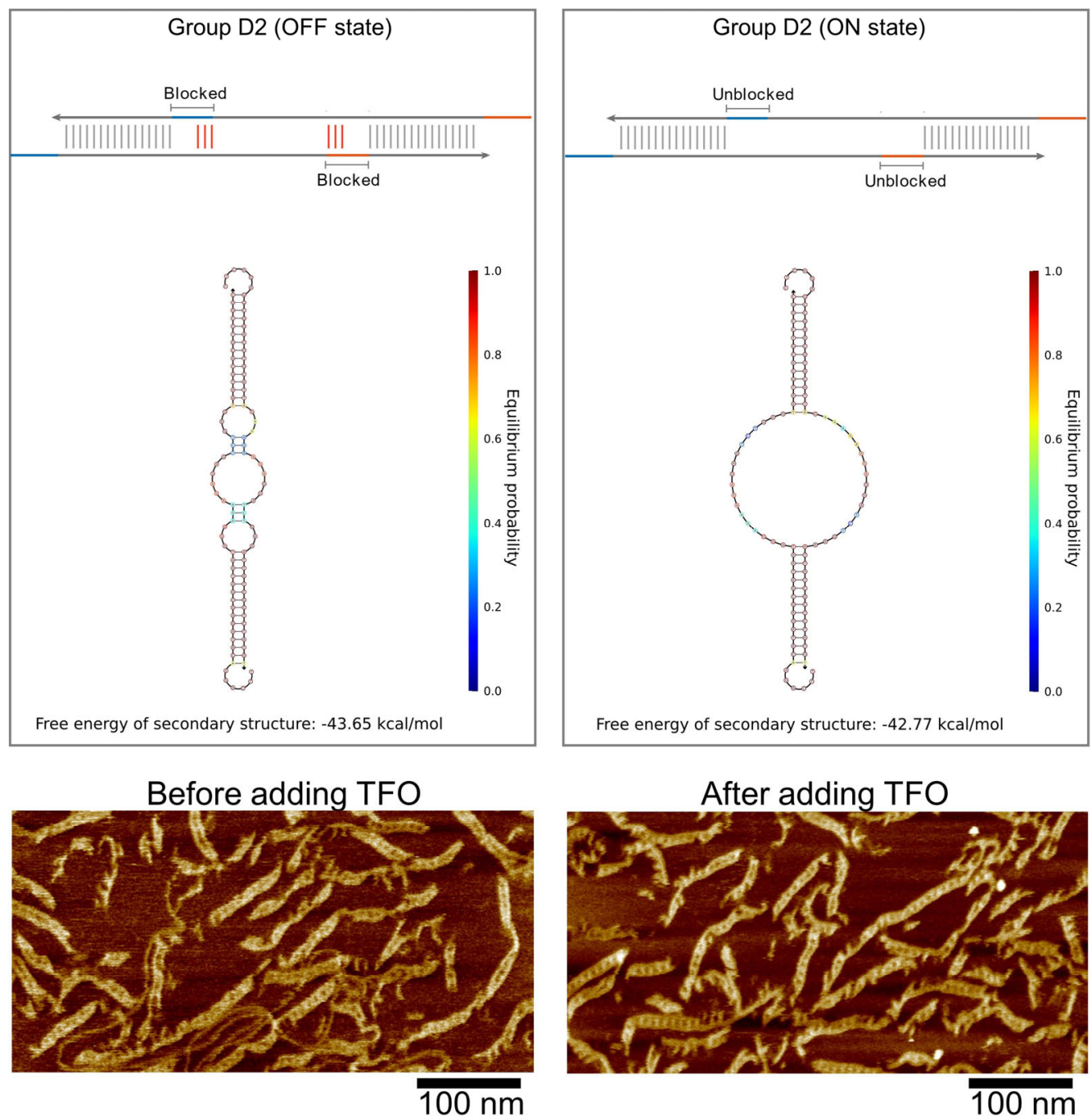

Figure S6. Schematic and simulated energetics parameter of the OFF state (left) and ON state (right) group D2 for two sites allosteric transition screening (top panel). AFM images of group D2 before (left) and after (right) adding TFO modulator (bottom panel). Scale bars, $100 \mathrm{~nm}$. 
2 sites allosteric transition screening
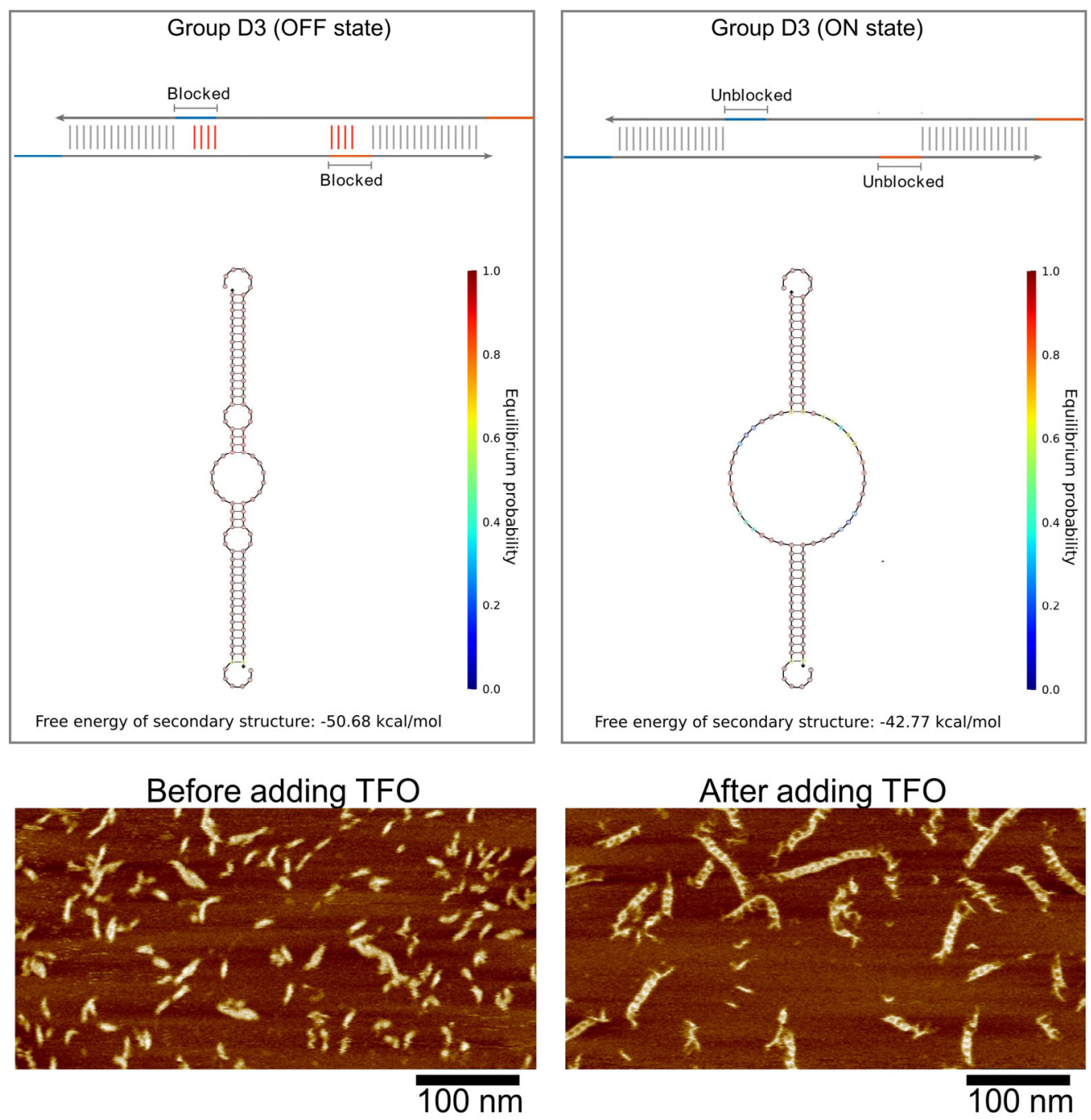

Figure S7. Schematic and simulated energetics parameter of the OFF state (left) and ON state (right) group D3 for two sites allosteric transition screening (top panel). AFM images of group D3 before (left) and after (right) adding TFO modulator (bottom panel). Scale bars, $100 \mathrm{~nm}$. 
2 sites allosteric transition screening
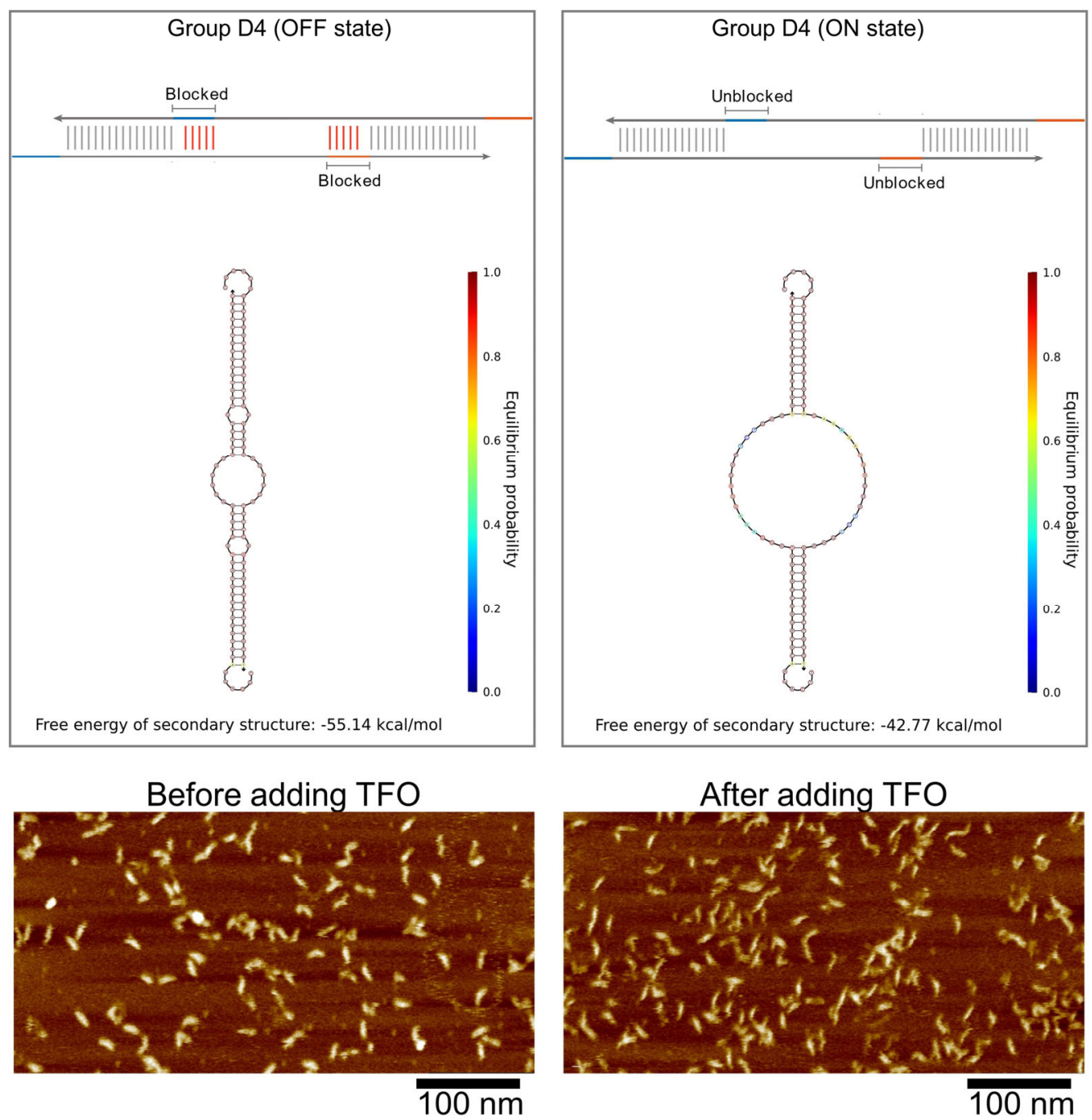

Figure S8. Schematic and simulated energetics parameter of the OFF state (left) and ON state (right) group D4 for two sites allosteric transition screening (top panel). AFM images of group D4 before (left) and after (right) adding TFO modulator (bottom panel). Scale bars, $100 \mathrm{~nm}$. 
2 sites allosteric transition screening
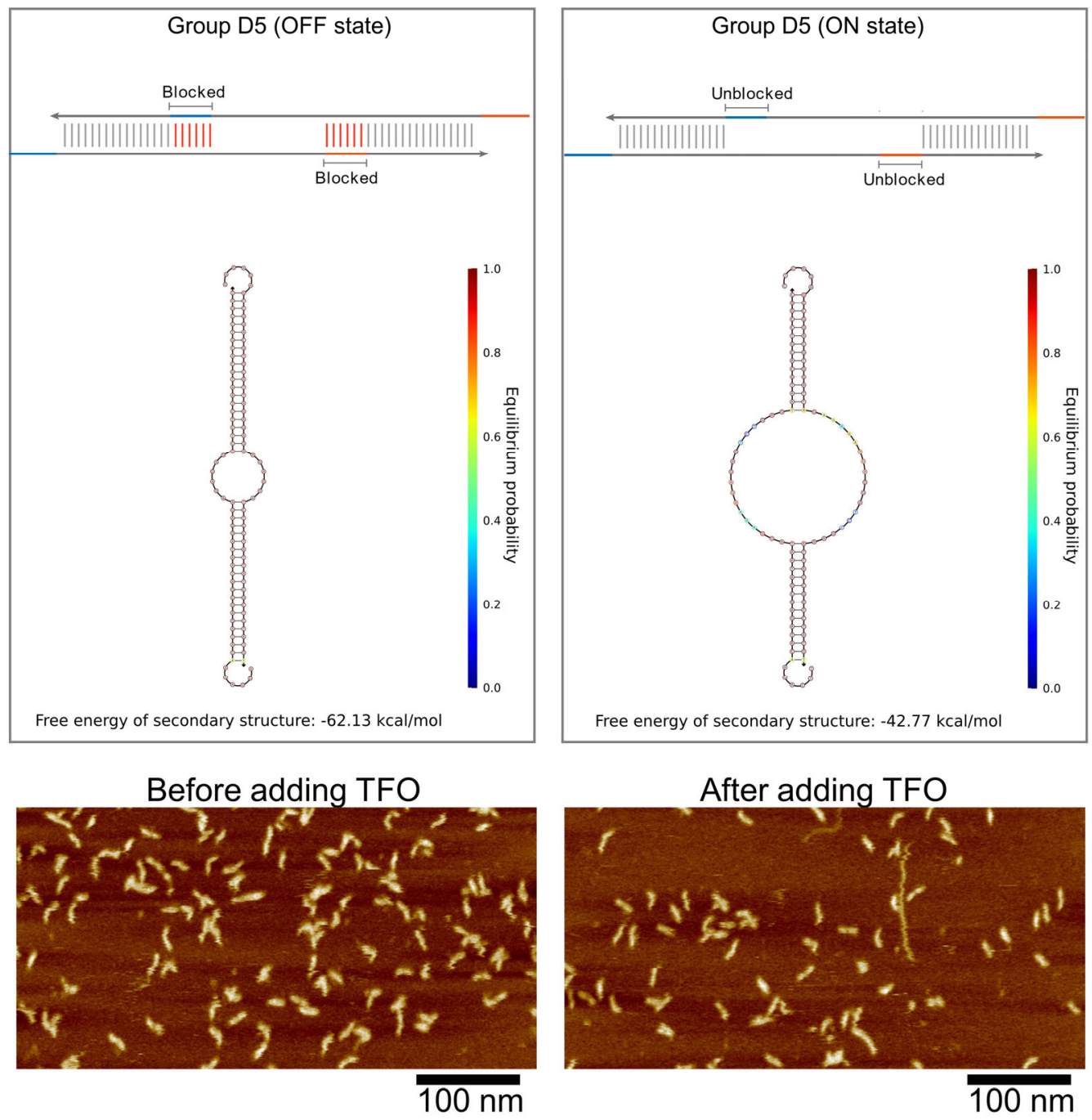

Figure S9. Schematic and simulated energetics parameter of the OFF state (left) and ON state (right) group D5 for two sites allosteric transition screening (top panel). AFM images of group D5 before (left) and after (right) adding TFO modulator (bottom panel). Scale bars, $100 \mathrm{~nm}$. 
2 sites allosteric transition screening
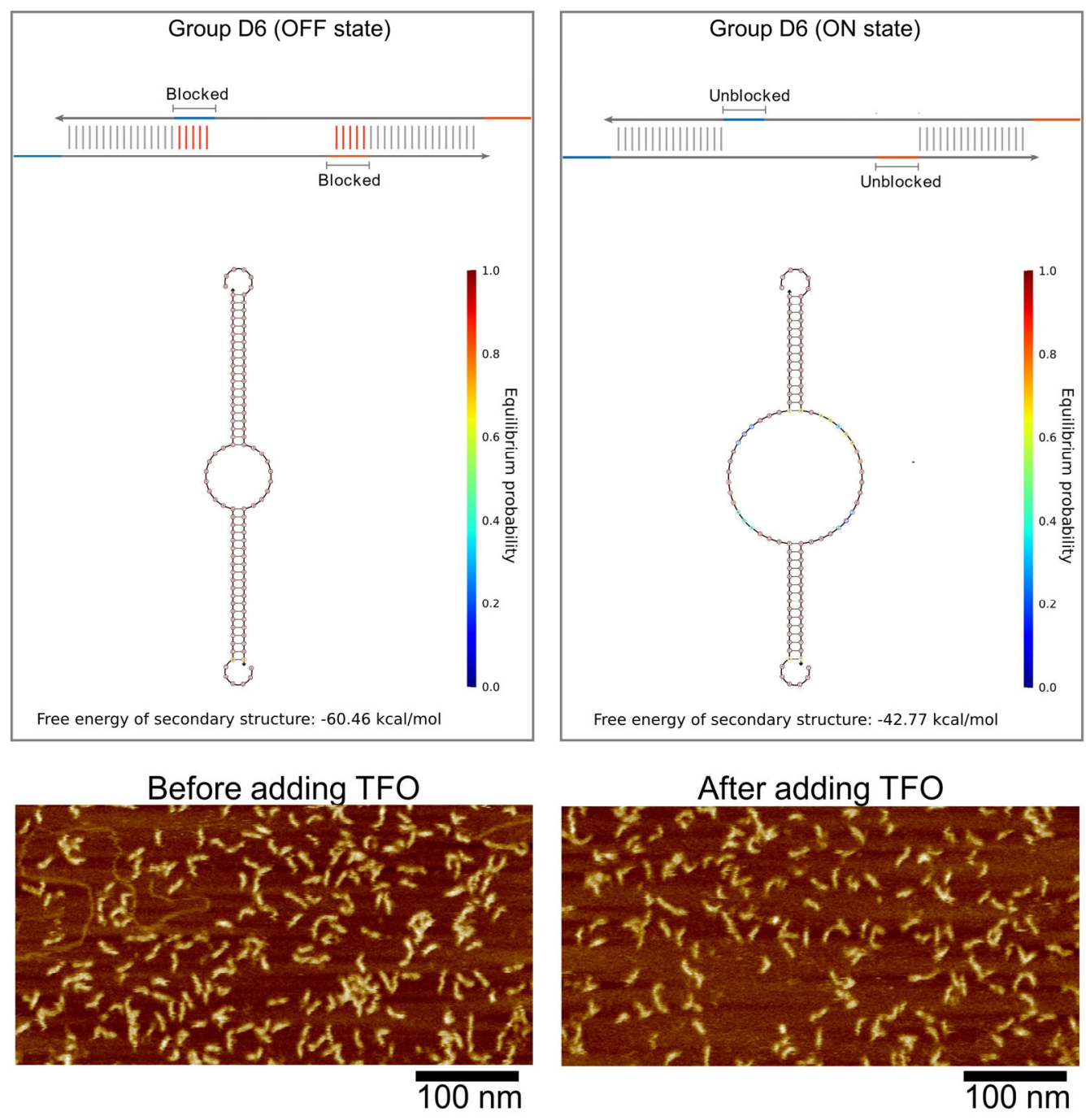

Figure S10. Schematic and simulated energetics parameter of the OFF state (left) and ON state (right) group D6 for two sites allosteric transition screening (top panel). AFM images of group D6 before (left) and after (right) adding TFO modulator (bottom panel). Scale bars, $100 \mathrm{~nm}$. 
2 sites allosteric transition screening
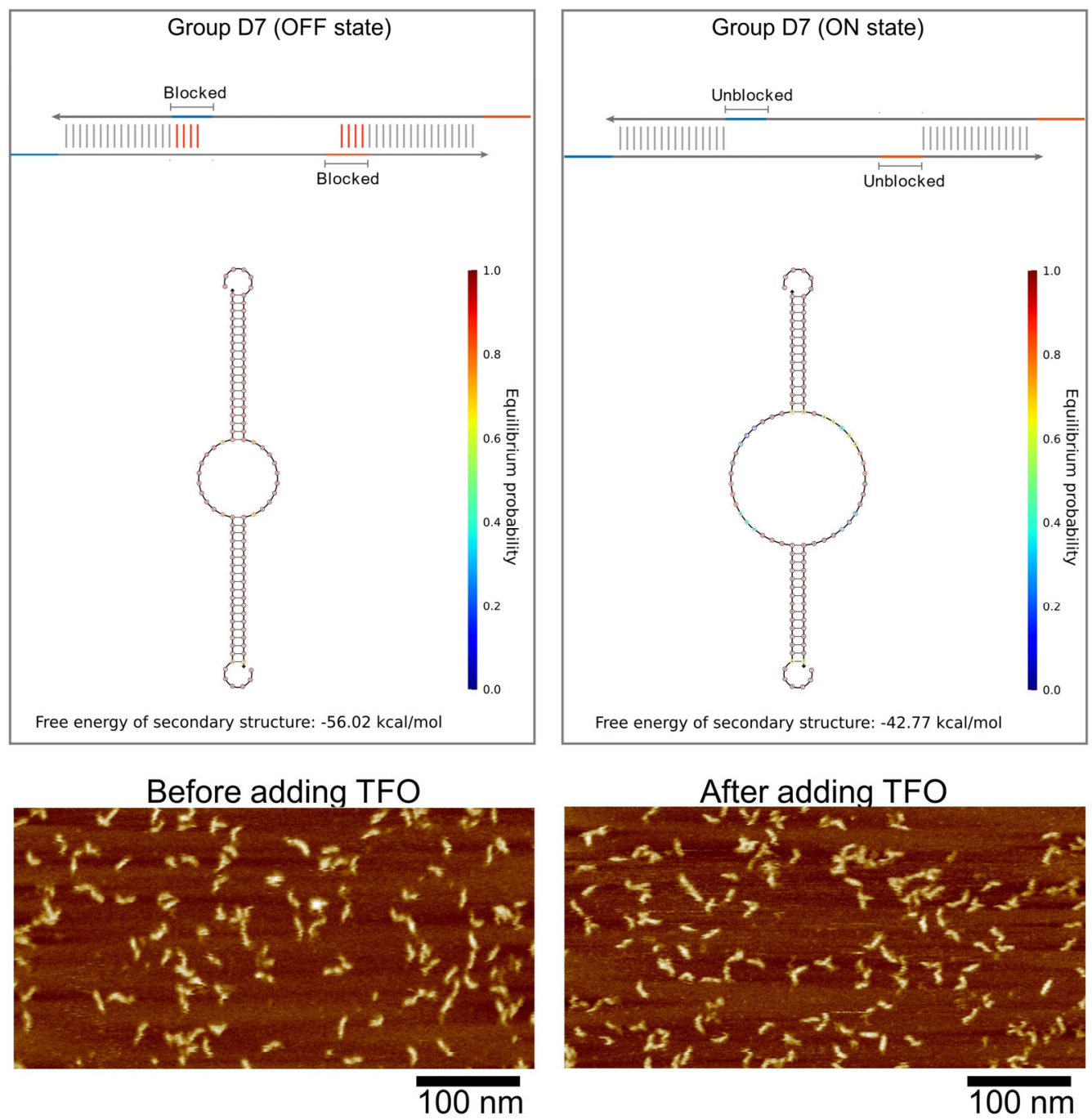

Figure S11. Schematic and simulated energetics parameter of the OFF state (left) and ON state (right) group D7 for two sites allosteric transition screening (top panel). AFM images of group D7 before (left) and after (right) adding TFO modulator (bottom panel). Scale bars, $100 \mathrm{~nm}$. 
2 sites allosteric transition screening
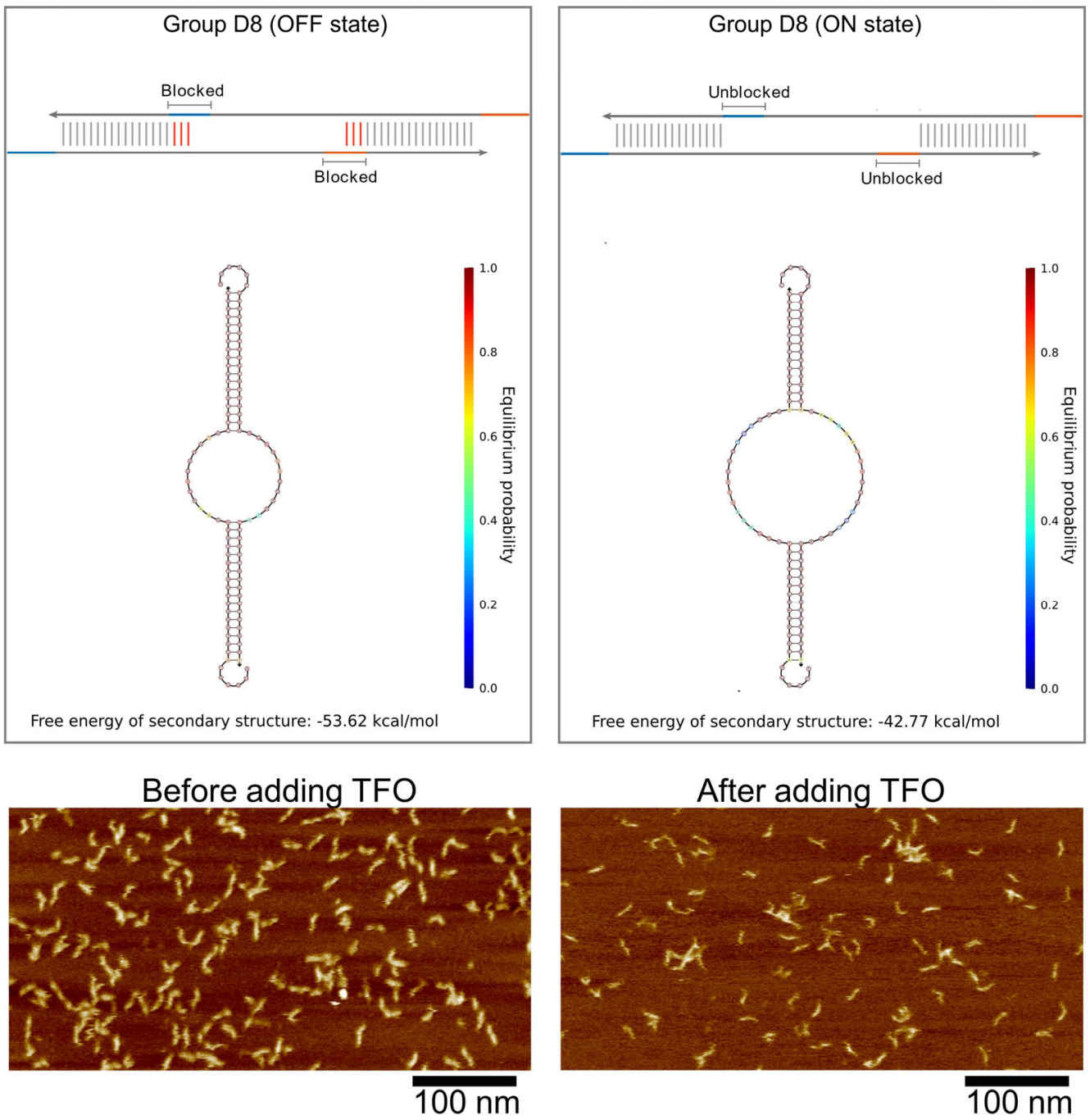

Figure S12. Schematic and simulated energetics parameter of the OFF state (left) and ON state (right) group D8 for two sites allosteric transition screening (top panel). AFM images of group D8 before (left) and after (right) adding TFO modulator (bottom panel). Scale bars, $100 \mathrm{~nm}$. 
2 sites allosteric transition screening
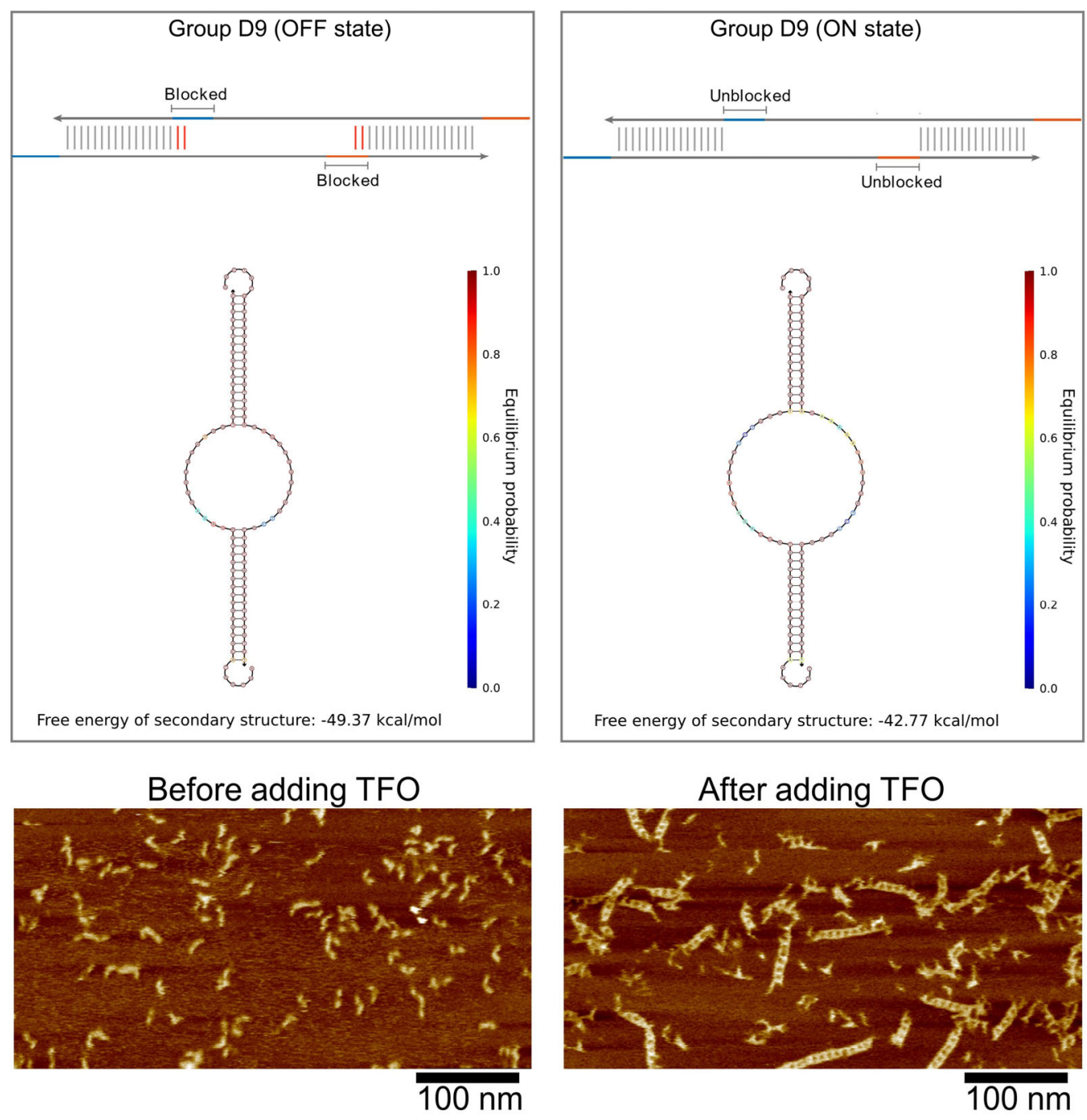

Figure S13. Schematic and simulated energetics parameter of the OFF state (left) and ON state (right) group D9 for two sites allosteric transition screening (top panel). AFM images of group D9 before (left) and after (right) adding TFO modulator (bottom panel). Scale bars, $100 \mathrm{~nm}$. 
2 sites allosteric transition screening
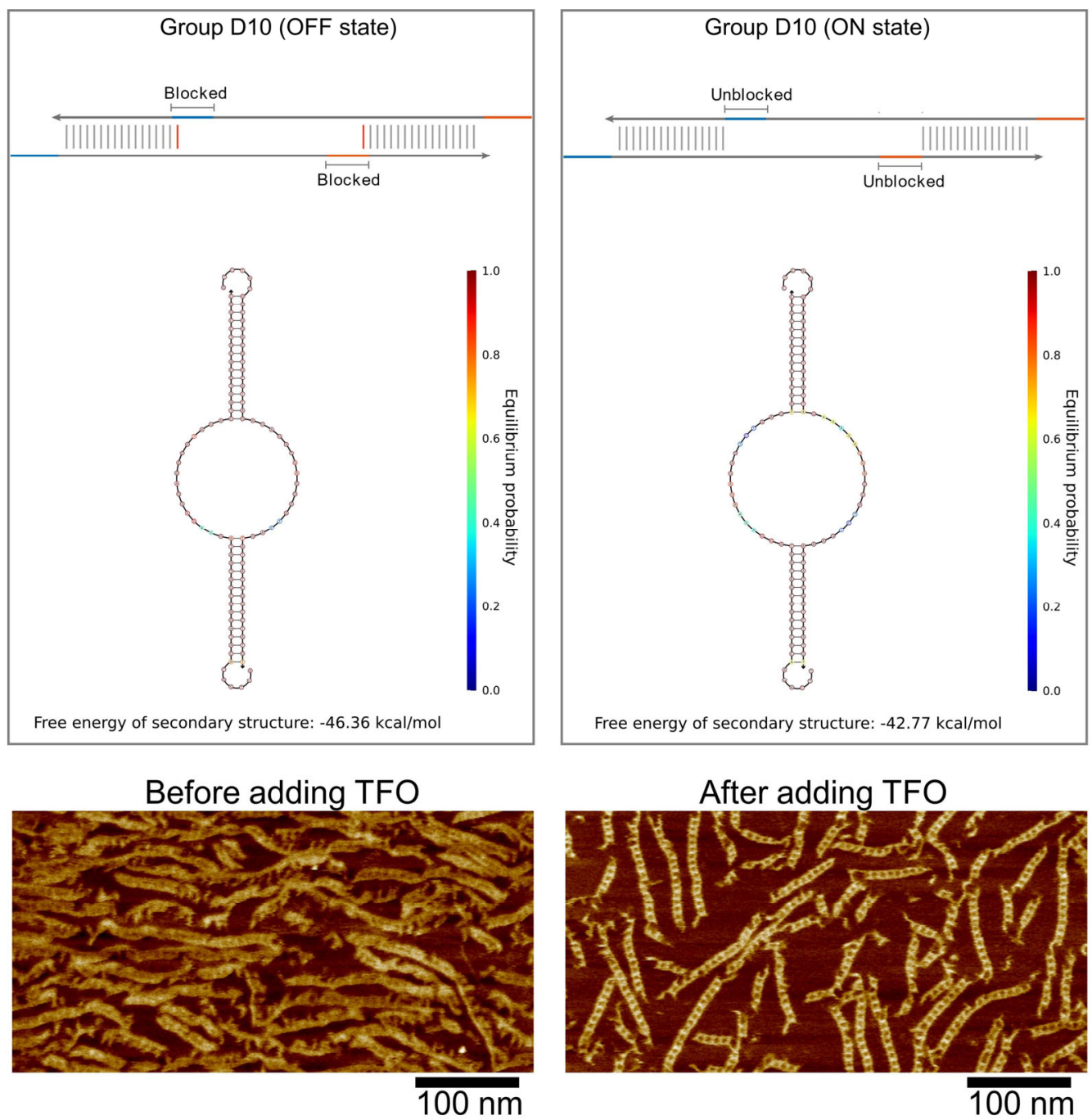

Figure S14. Schematic and simulated energetics parameter of the OFF state (left) and ON state (right) group D10 for two sites allosteric transition screening (top panel). AFM images of group D10 before (left) and after (right) adding TFO modulator (bottom panel). Scale bars, $100 \mathrm{~nm}$. 
2 sites allosteric transition screening
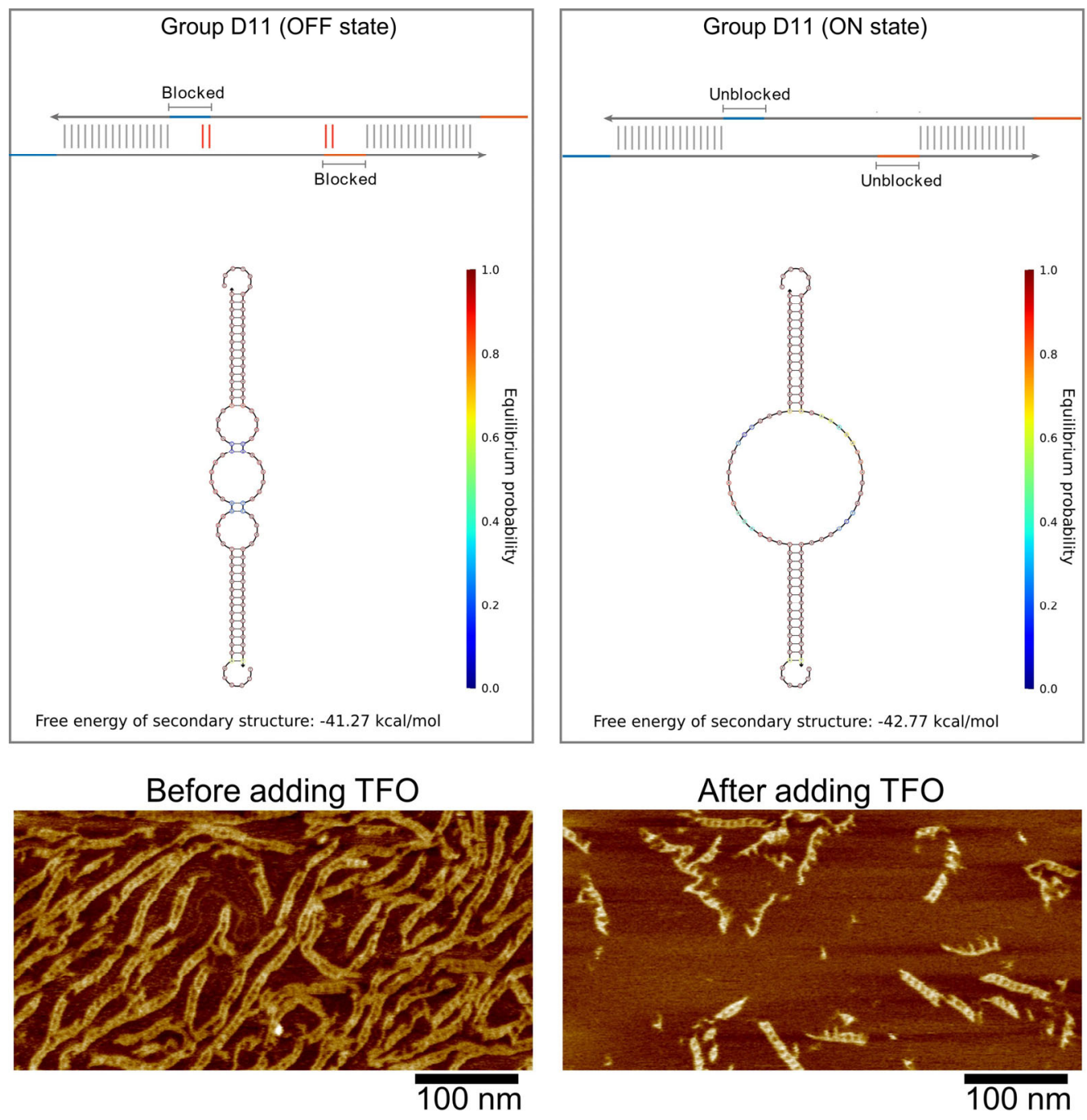

Figure S15. Schematic and simulated energetics parameter of the OFF state (left) and ON state (right) group D11 for two sites allosteric transition screening (top panel). AFM images of group D11 before (left) and after (right) adding TFO modulator (bottom panel). Scale bars, $100 \mathrm{~nm}$. 
2 sites allosteric transition screening
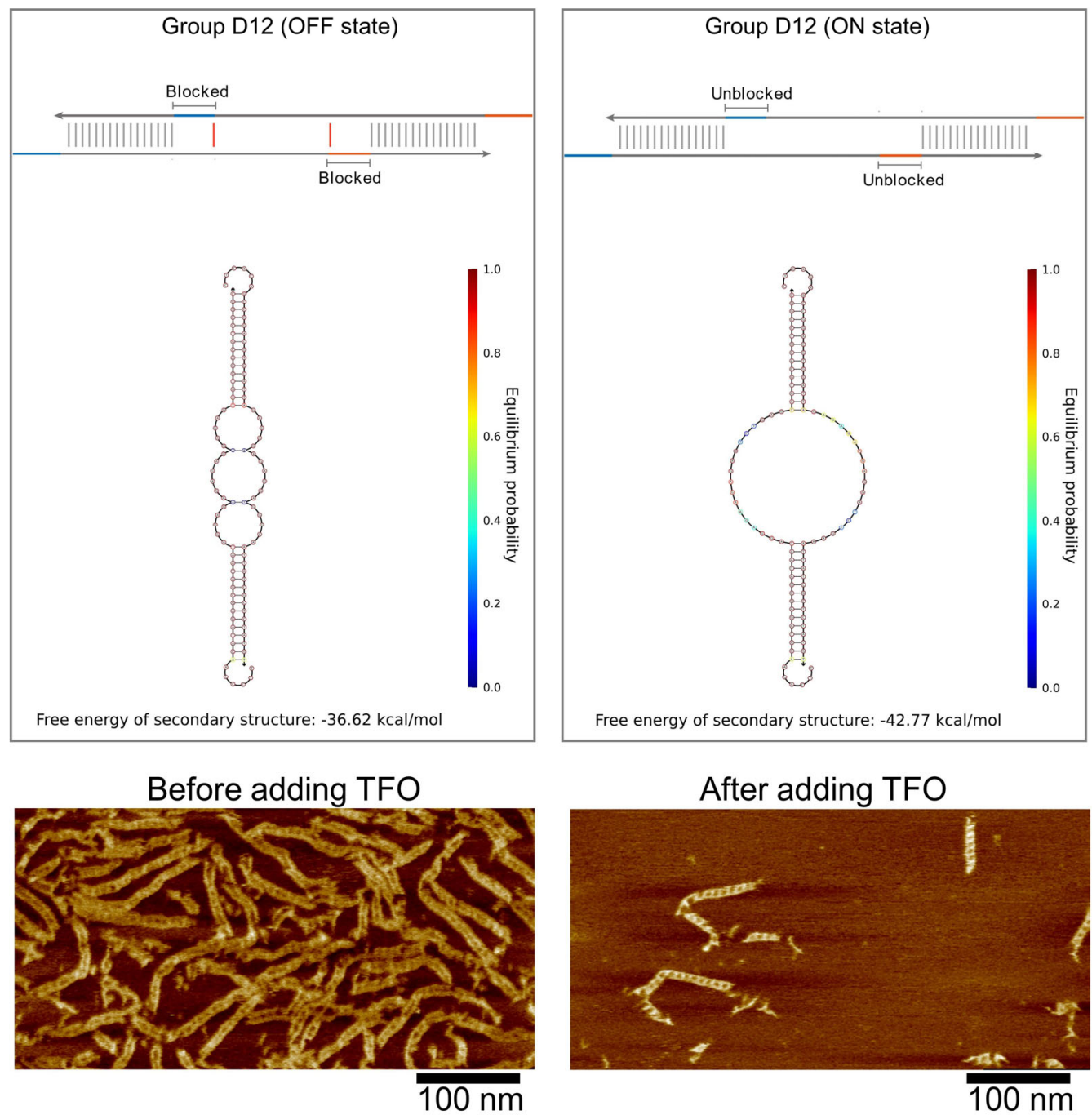

Figure S16. Schematic and simulated energetics parameter of the OFF state (left) and ON state (right) group D12 for two sites allosteric transition screening (top panel). AFM images of group D12 before (left) and after (right) adding TFO modulator (bottom panel). Scale bars, $100 \mathrm{~nm}$. 


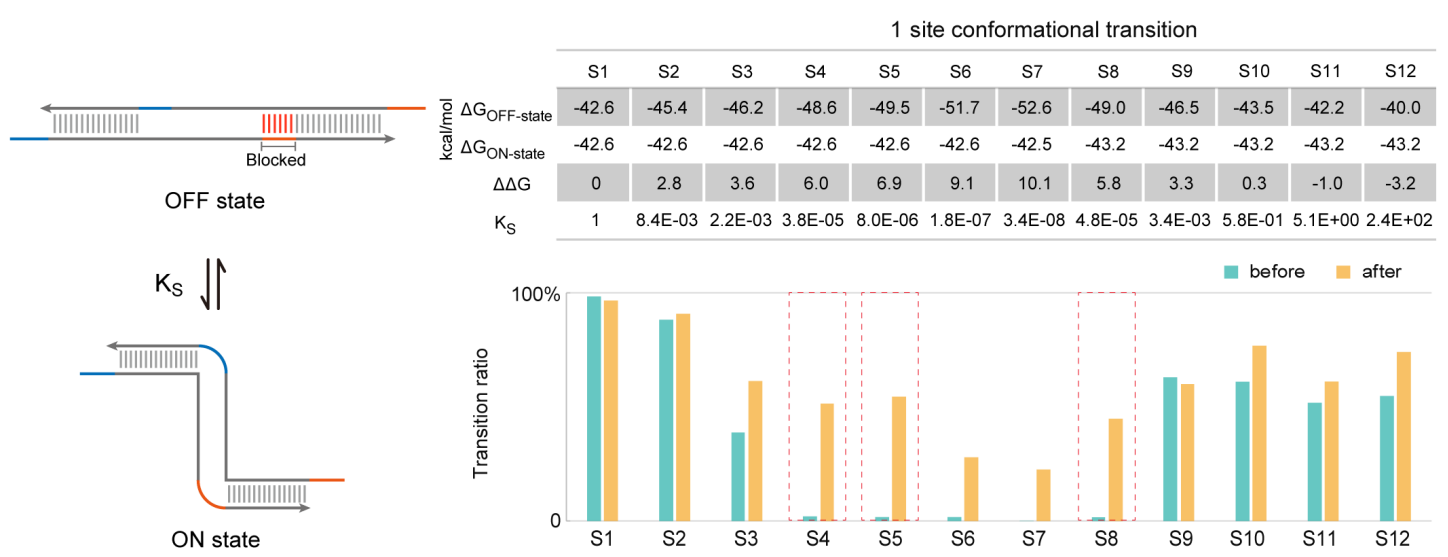

Figure S17. Design and screening of DNA triplex-based allosteric nanodevices with one site conformational transition. a, Design schematic showing the blocked active sites (OFF state) and open active sites (ON state) in triplex-based Z-switch without TFO modulator. Blue and orange: active sites that can interact with $5^{\prime}$ overhangs of the same color; red lines: block the active sites using hydrogen bonds. $\mathbf{b}$, NUPACK simulations showing energetics parameters of 12 experimental groups with different levels of blocking. c, Calculated transition ratio of 12 experimental groups. Green: before adding TFO modulators; yellow: after adding TFO modulators. See Table S3 for data collection.

Because the other active site was not treated and can directly bind to the corresponding $5^{\prime}$ free end of another Z-switch, the binding of this untreated active site will cause a cooperative effect and result in a compensation to the energy barrier of the ON-OFF transition of the treated site. Under this circumstance, the desired variants were estimated to have more stable block on the treated site to maintain a clear-off OFF state that comparable with double sites treatment. Three variants (S4, S5 and S8) were selected out on account of the apparent transition from comb polymers to ladder polymers upon TFO modulator binding. Consistent with the anticipation, the treated site of each variant took a bigger $\triangle \mathrm{G}$ to exclude the cooperation effect (i.e., for the single treated site, $\mathrm{S} 4 / \mathrm{S} 5 / \mathrm{S} 8$ has a $\triangle \mathrm{G}$ of $\sim 6 \mathrm{kcal} / \mathrm{mol}$ while $\mathrm{D} 3 / \mathrm{D} 9$ has a $\triangle \mathrm{G}$ of $\sim 4$ (half of 7.9) $\mathrm{kcal} / \mathrm{mol}$ ) and finally maintain the same Ks values $\left(\sim 10^{-6}\right)$. As a conclusion, the clear-off ON-OFF transition were successfully achieved by designing and screening a series of pre-set blocking of different levels. While no ON-OFF transition was presented for nine negative variants, variants with Ks values $>10^{-5}$ (S1, S2, S3, S9, S10, $\mathrm{S} 11, \mathrm{~S} 12)$ displayed failed blocking on the active sites, resulting in ambiguous ON-OFF transition states. While variants with Ks values $<10^{-6}$ (S6 and S7) were incapable to be regulated by TFO modulator due to the high energy barrier of blocked active sites. 
d

a

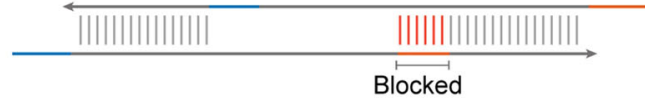

b

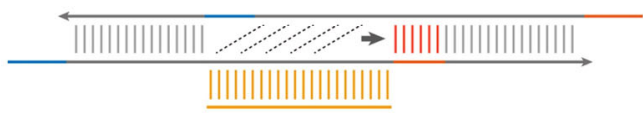

Triplex formation propagation

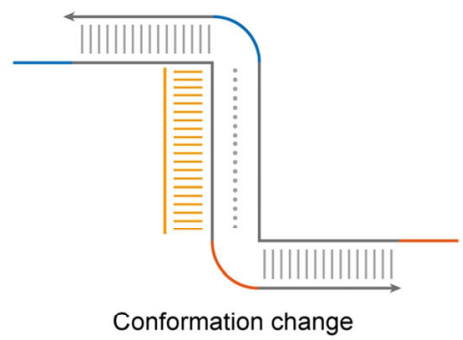

C

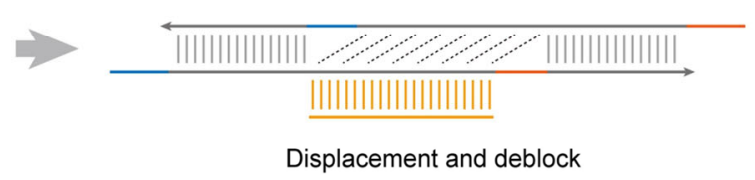

Figure S18. Allosteric transition of triplex-induced Z-switches with 1 site blocked. a, The blocked state of Z-switch. b, Triplex formation and propagation. c, Triplex propagation induces strand displacement and reopens the blocked site. $\mathbf{d}$, The ON state after triplex-induced conformational change. 


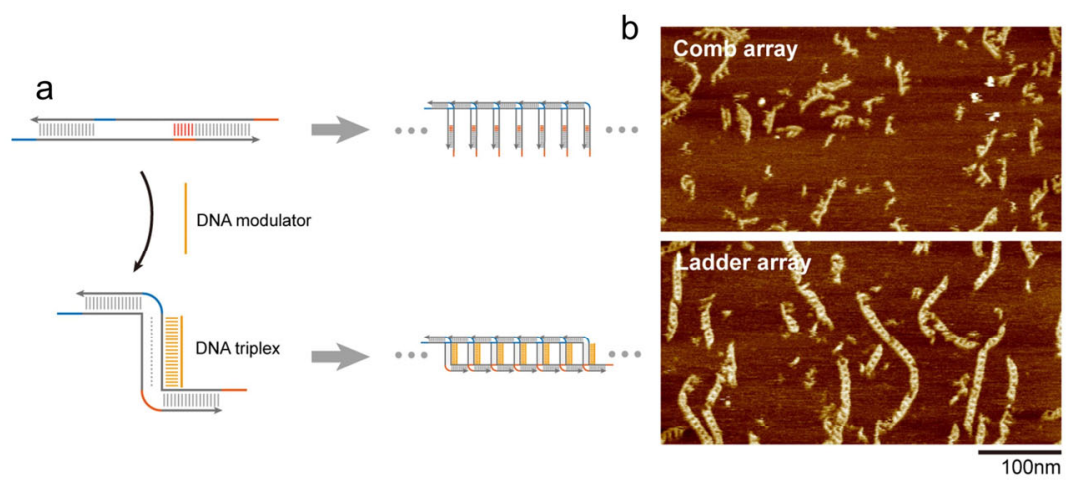

Figure S19. a, Schematic showing the conformational transition upon TFO modulator binding and the transition from OFF state to ON state where OFF-state switch can assemble into comb array and $\mathrm{ON}$-state switch can further assemble into ladder array. b, AFM images showing the comb array and ladder array. Scale bars, $100 \mathrm{~nm}$. 


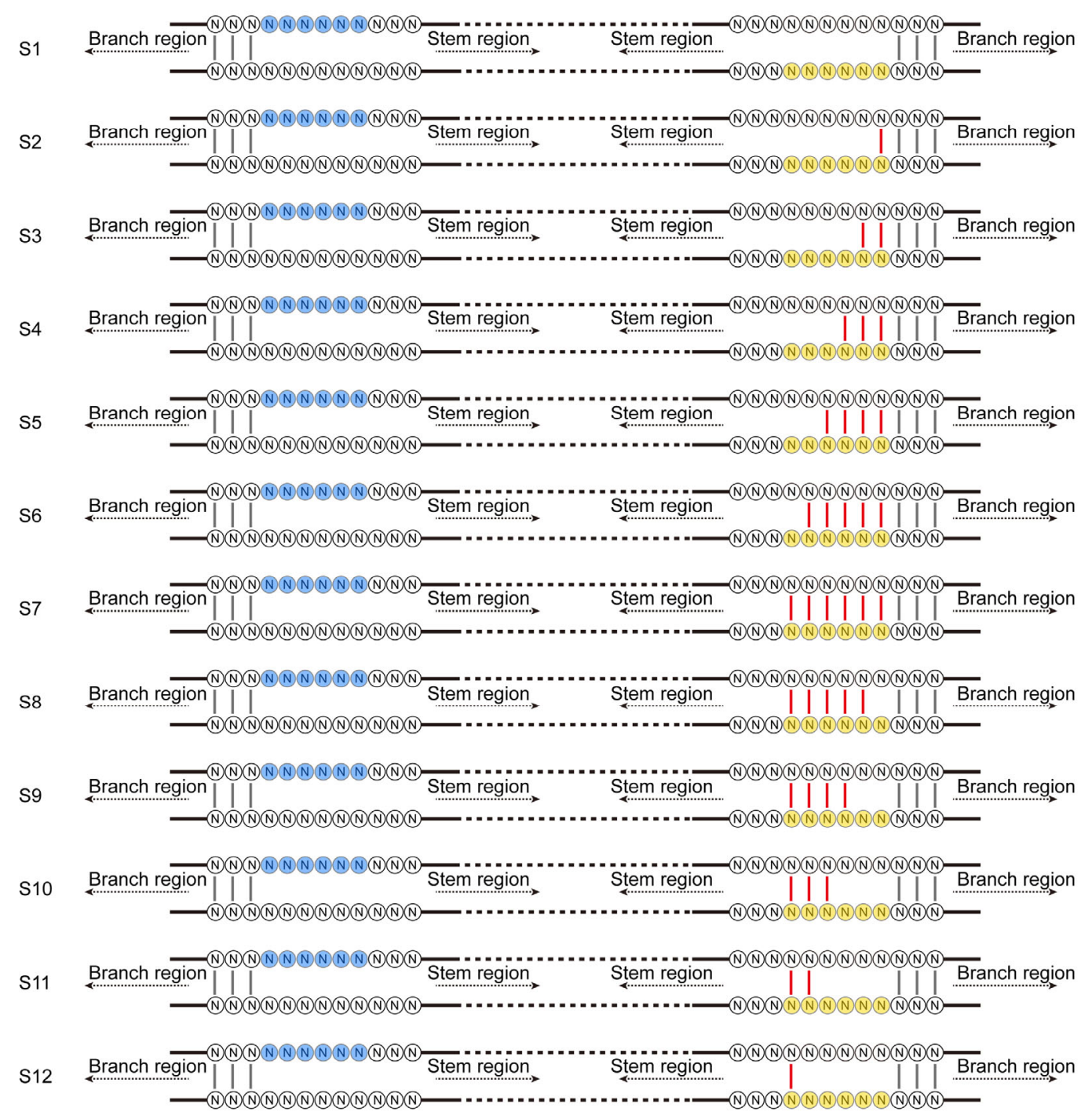

Figure S20. Schematic showing the allosteric Z-switches with 12 different levels of blocking on the right active site (site $\left.b^{*}\right)$. Blue region represents active site $a^{*}$ and yellow region represents active site $b^{*} .5$ ' overhangs are not displayed. All nucleic acids are marked as $\mathbf{N}$ and vertical lines represent the hydrogen bond. Red vertical lines represent the blocking on active site $b^{*}$. 
1 site allosteric transition screening
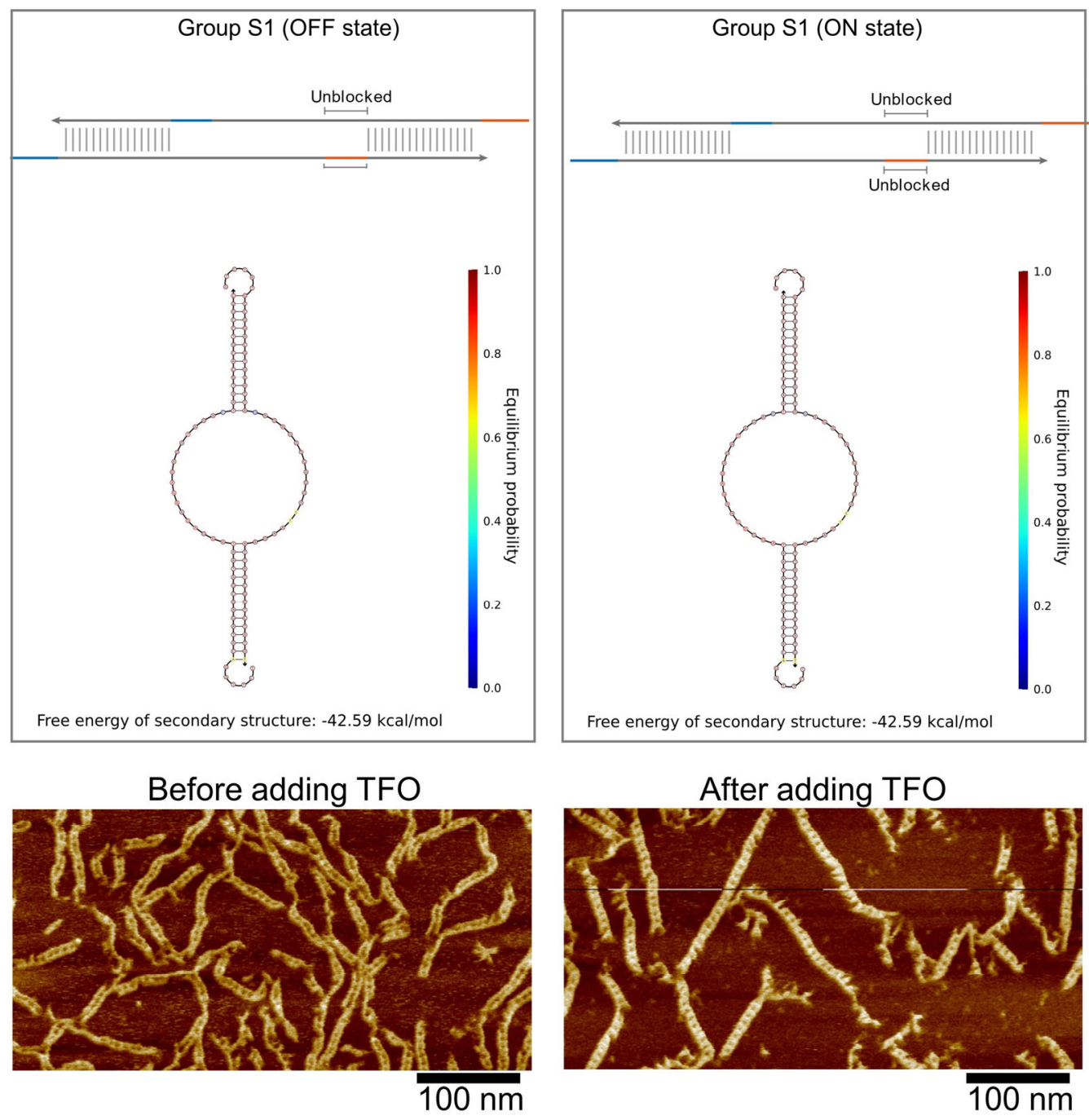

Figure S21. Schematic and simulated energetics parameter of group S1 for one site allosteric transition screening (left panel). AFM images of group S1 before and after adding TFO modulator (right panel). Scale bars, $100 \mathrm{~nm}$. 
1 site allosteric transition screening
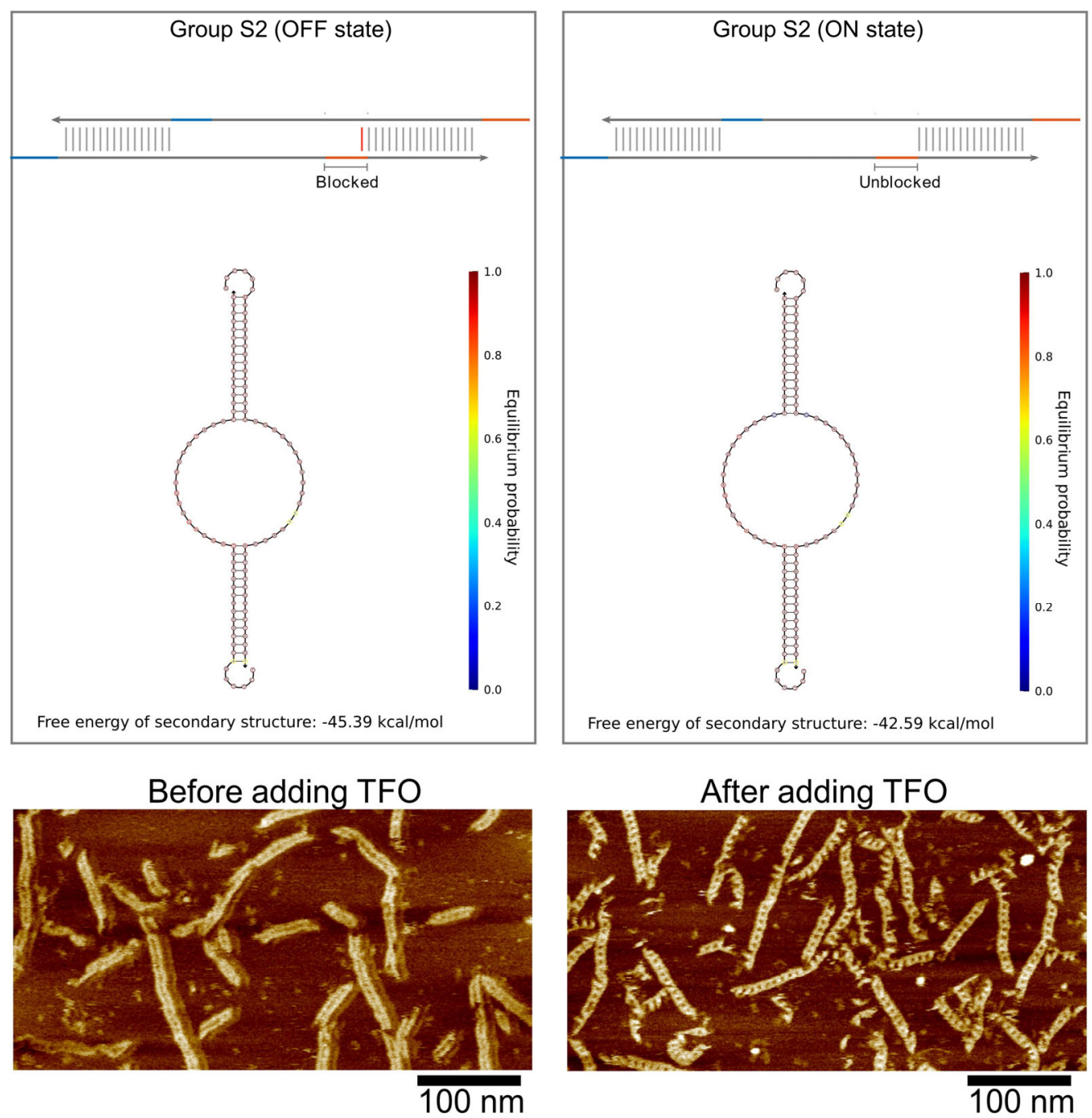

Figure S22. Schematic and simulated energetics parameter of the OFF state (left) and ON state (right) group S2 for one site allosteric transition screening (top panel). AFM images of group S2 before (left) and after (right) adding TFO modulator (bottom panel). Scale bars, $100 \mathrm{~nm}$. 
1 site allosteric transition screening
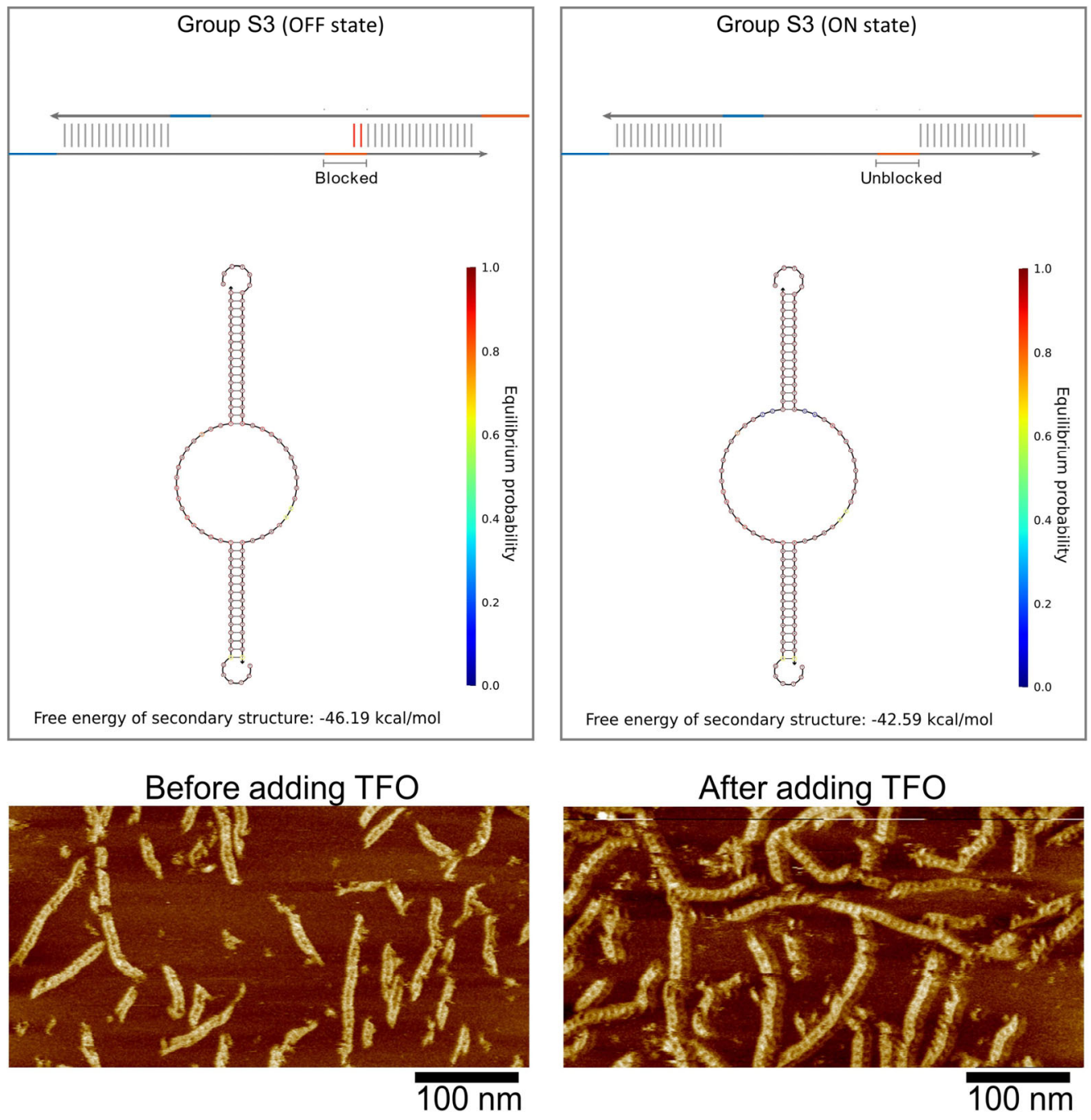

Figure S23. Schematic and simulated energetics parameter of the OFF state (left) and ON state (right) group S3 for one site allosteric transition screening (top panel). AFM images of group S3 before (left) and after (right) adding TFO modulator (bottom panel). Scale bars, $100 \mathrm{~nm}$. 
1 site allosteric transition screening
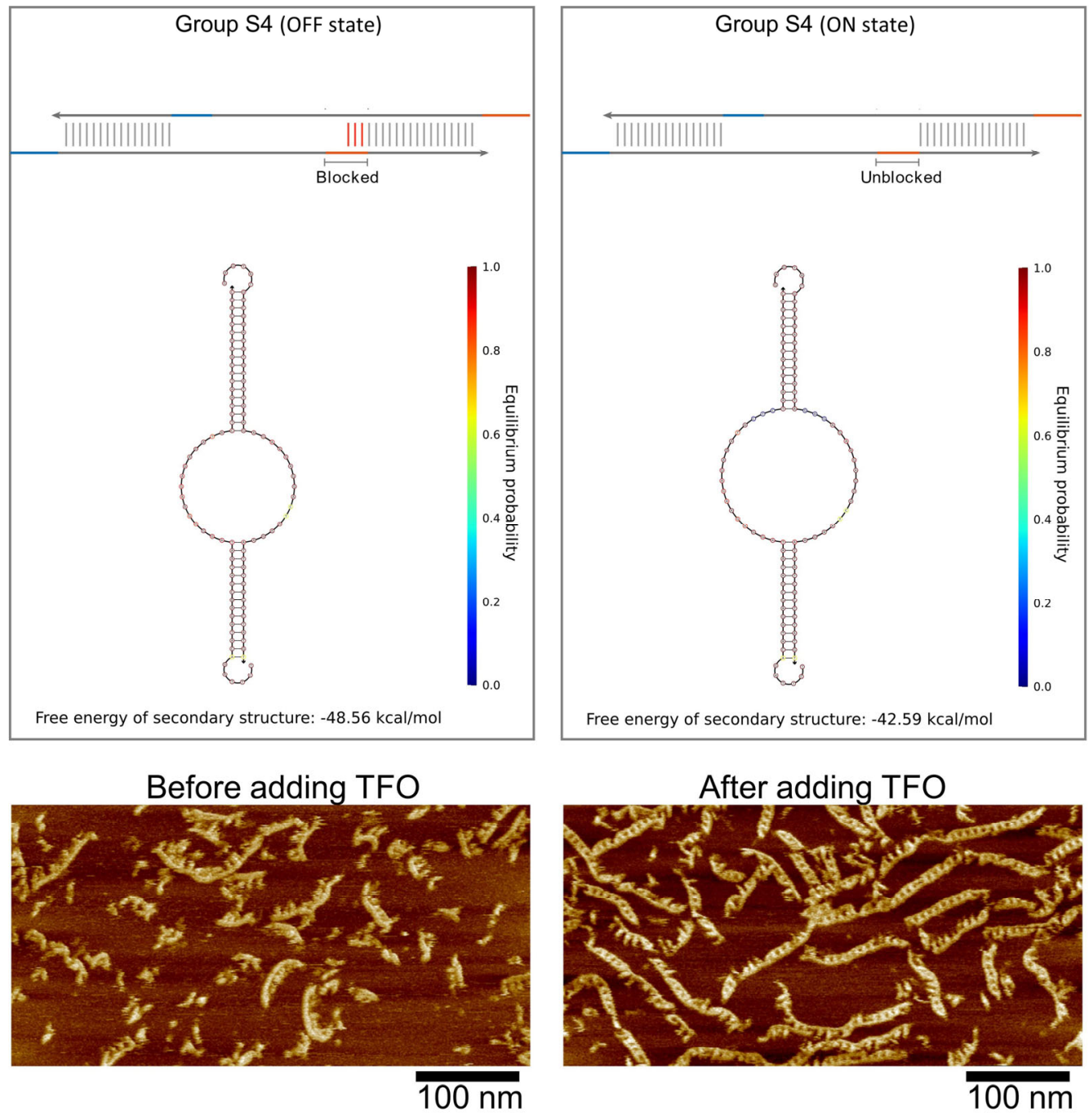

Figure S24. Schematic and simulated energetics parameter of the OFF state (left) and ON state (right) group S4 for one site allosteric transition screening (top panel). AFM images of group S4 before (left) and after (right) adding TFO modulator (bottom panel). Scale bars, $100 \mathrm{~nm}$. 
1 site allosteric transition screening
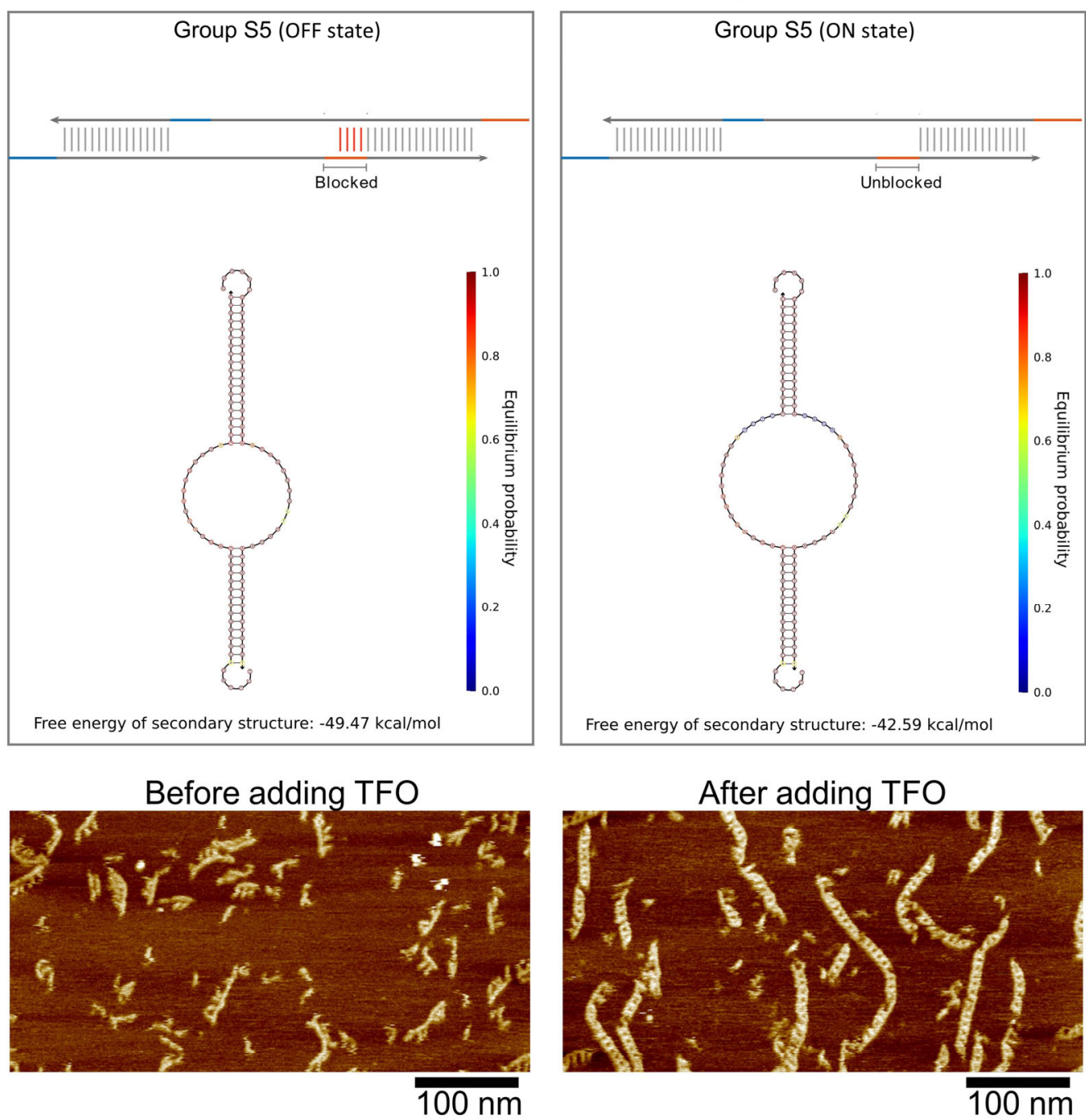

Figure S25. Schematic and simulated energetics parameter of the OFF state (left) and ON state (right) group S5 for one site allosteric transition screening (top panel). AFM images of group S5 before (left) and after (right) adding TFO modulator (bottom panel). Scale bars, $100 \mathrm{~nm}$. 
1 site allosteric transition screening
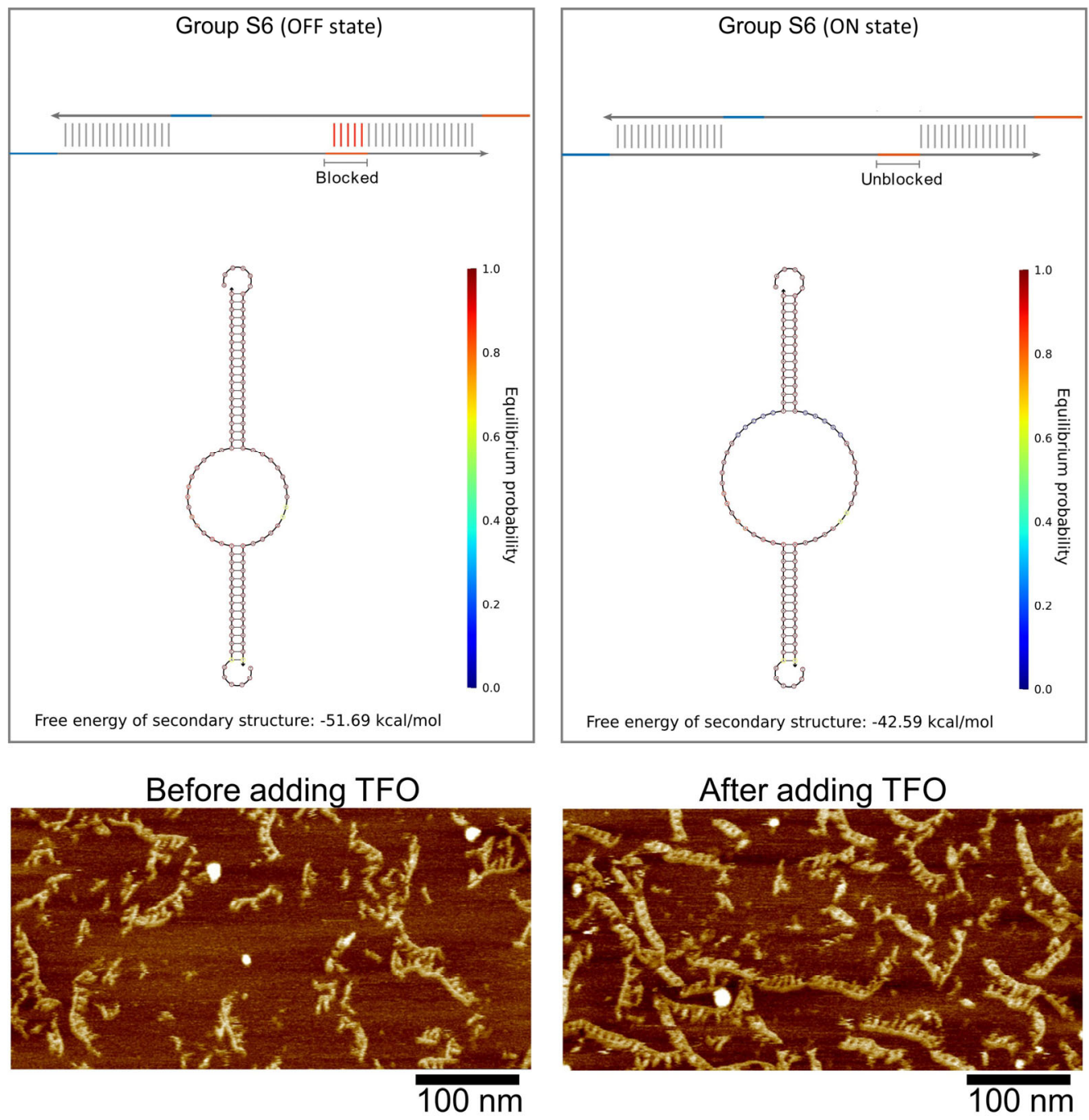

Figure S26. Schematic and simulated energetics parameter of the OFF state (left) and ON state (right) group S6 for one site allosteric transition screening (top panel). AFM images of group S6 before (left) and after (right) adding TFO modulator (bottom panel). Scale bars, $100 \mathrm{~nm}$. 
1 site allosteric transition screening
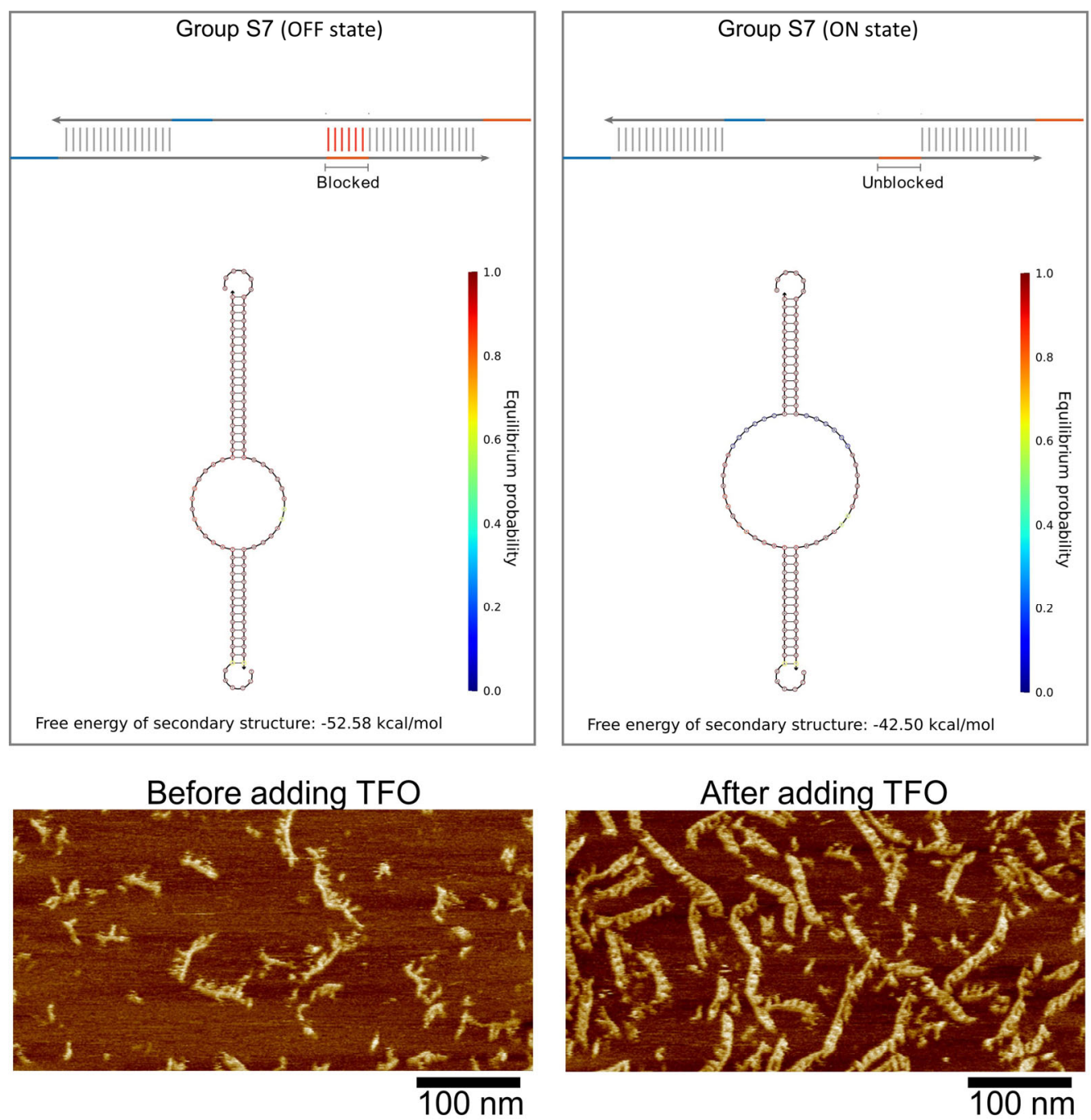

Figure S27. Schematic and simulated energetics parameter of the OFF state (left) and ON state (right) group S7 for one site allosteric transition screening (top panel). AFM images of group S7 before (left) and after (right) adding TFO modulator (bottom panel). Scale bars, $100 \mathrm{~nm}$. 
1 site allosteric transition screening

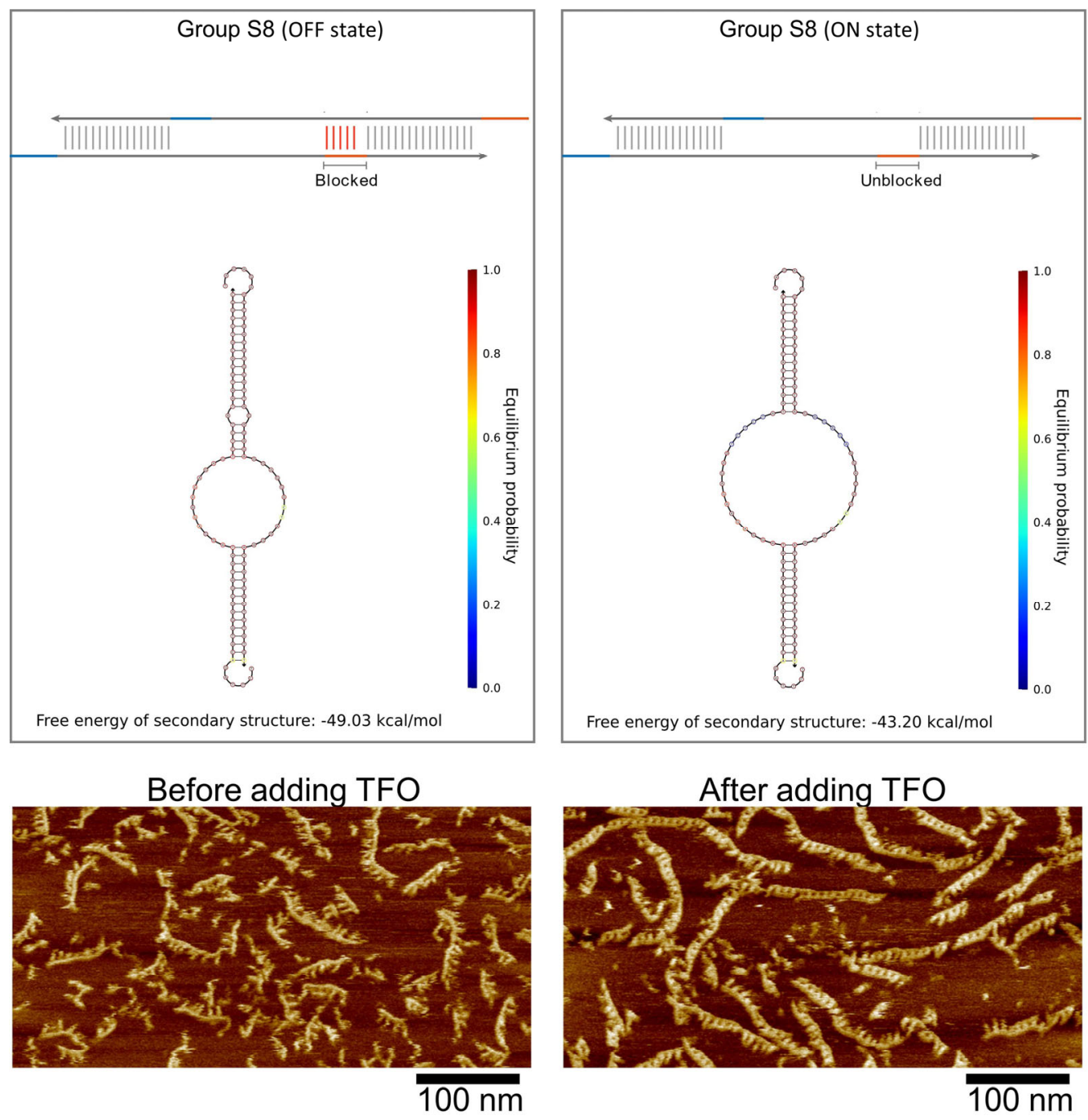

Figure S28. Schematic and simulated energetics parameter of the OFF state (left) and ON state (right) group S8 for one site allosteric transition screening (top panel). AFM images of group S8 before (left) and after (right) adding TFO modulator (bottom panel). Scale bars, $100 \mathrm{~nm}$. 
1 site allosteric transition screening
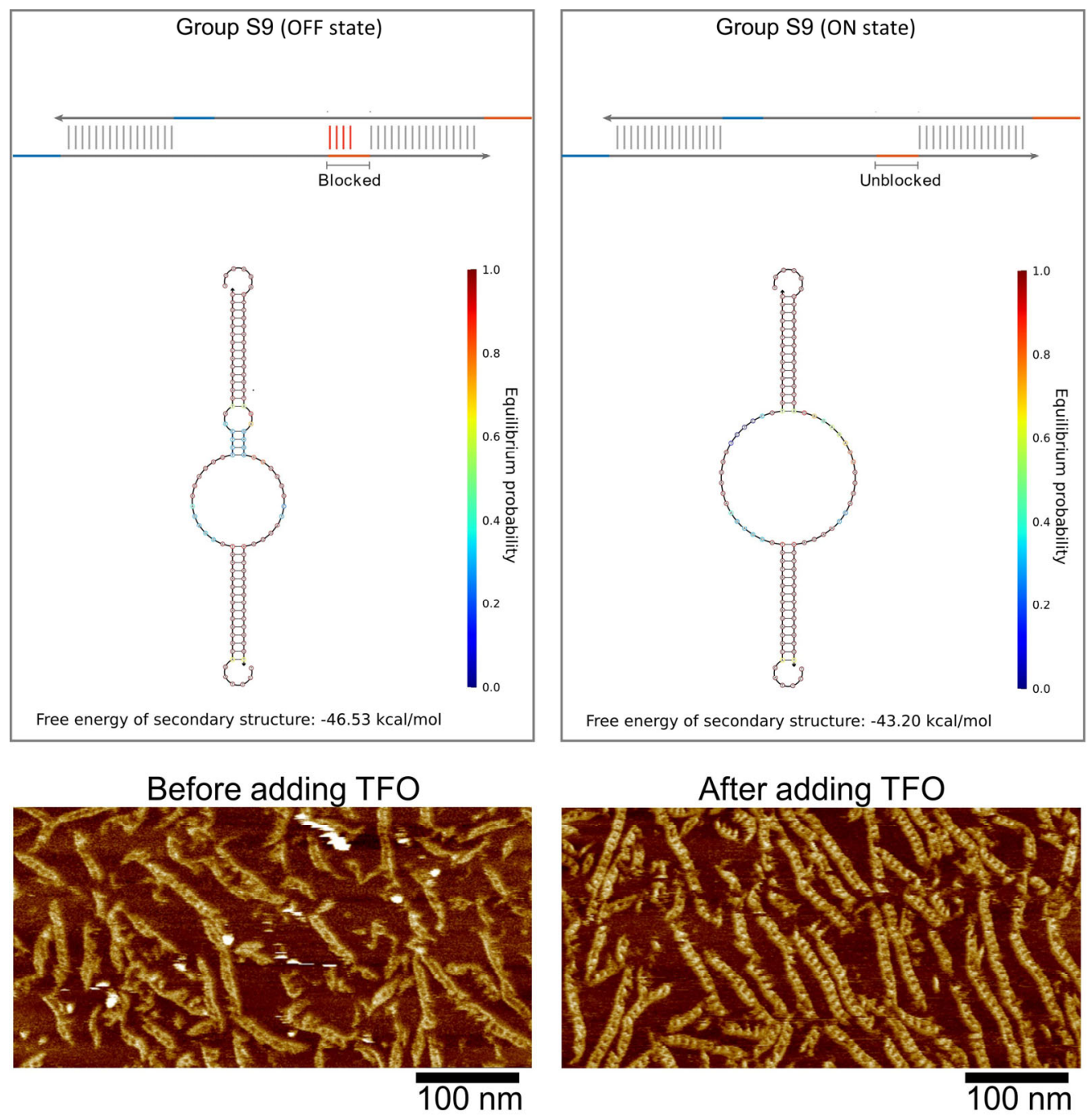

Figure S29. Schematic and simulated energetics parameter of the OFF state (left) and ON state (right) group S9 for one site allosteric transition screening (top panel). AFM images of group S9 before (left) and after (right) adding TFO modulator (bottom panel). Scale bars, $100 \mathrm{~nm}$. 
1 site allosteric transition screening
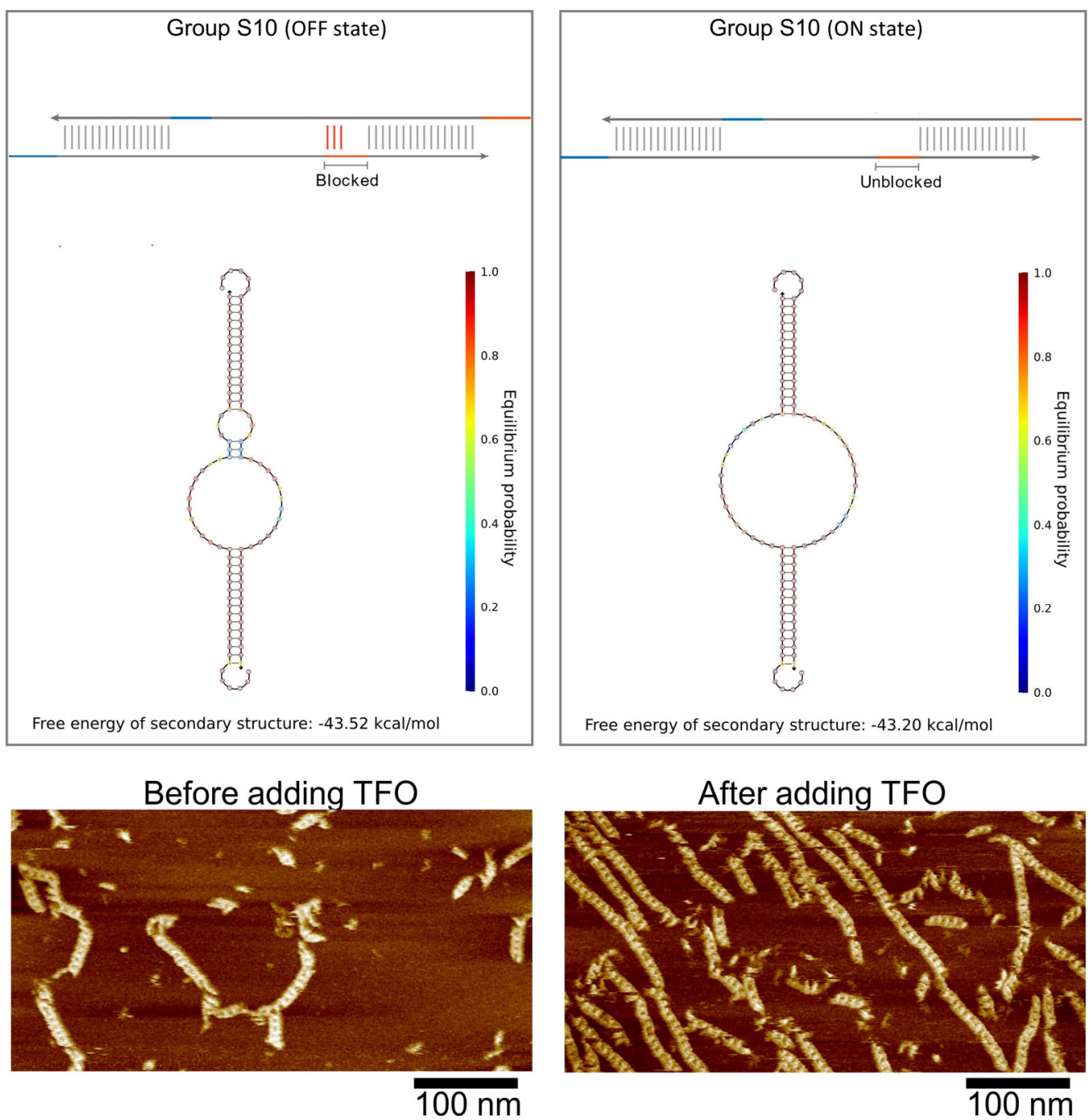

Figure S30. Schematic and simulated energetics parameter of the OFF state (left) and ON state (right) group S10 for one site allosteric transition screening (top panel). AFM images of group S10 before (left) and after (right) adding TFO modulator (bottom panel). Scale bars, $100 \mathrm{~nm}$. 
1 site allosteric transition screening
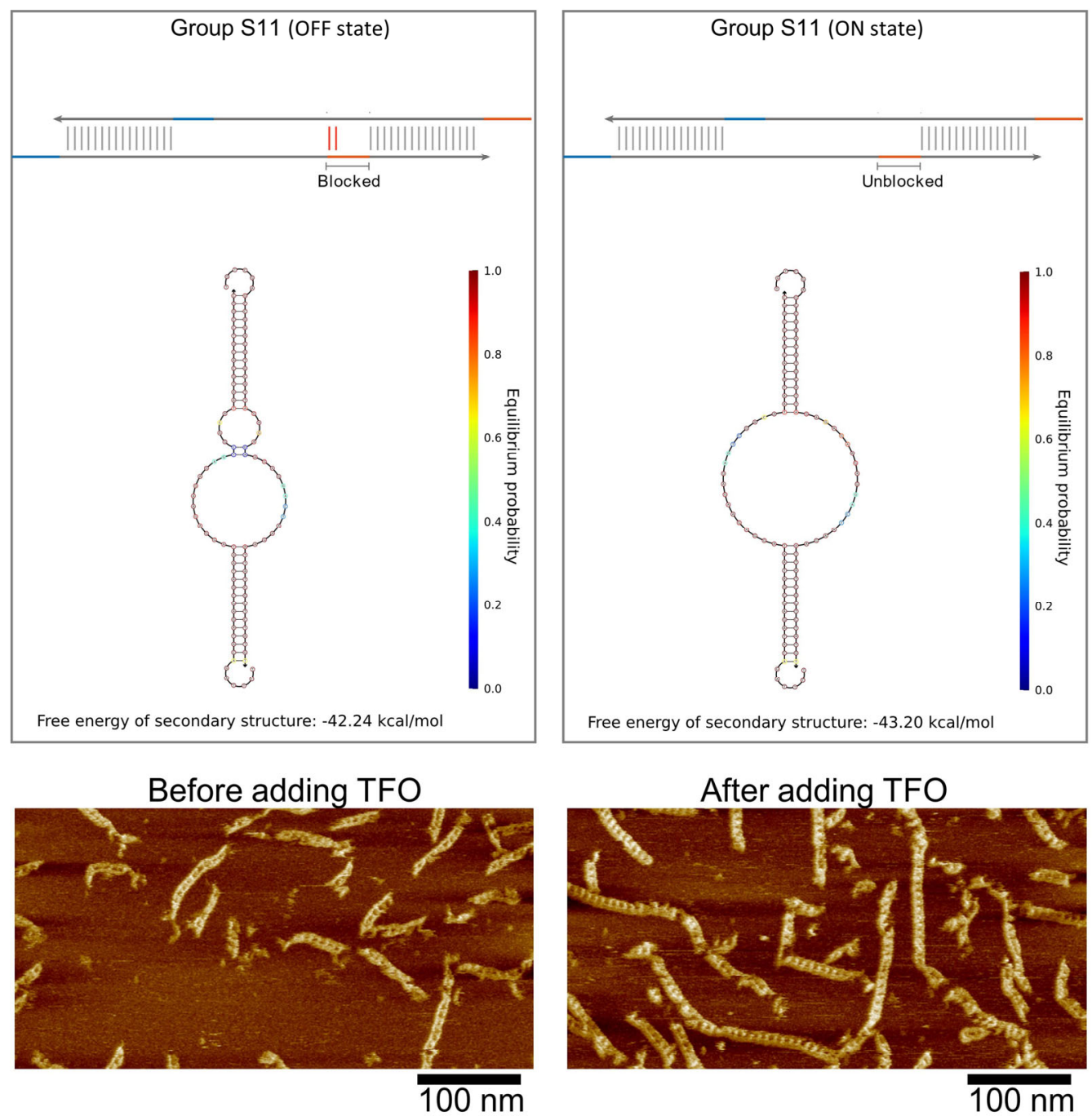

Figure S31. Schematic and simulated energetics parameter of the OFF state (left) and ON state (right) group S11 for one site allosteric transition screening (top panel). AFM images of group S11 before (left) and after (right) adding TFO modulator (bottom panel). Scale bars, $100 \mathrm{~nm}$. 
1 site allosteric transition screening
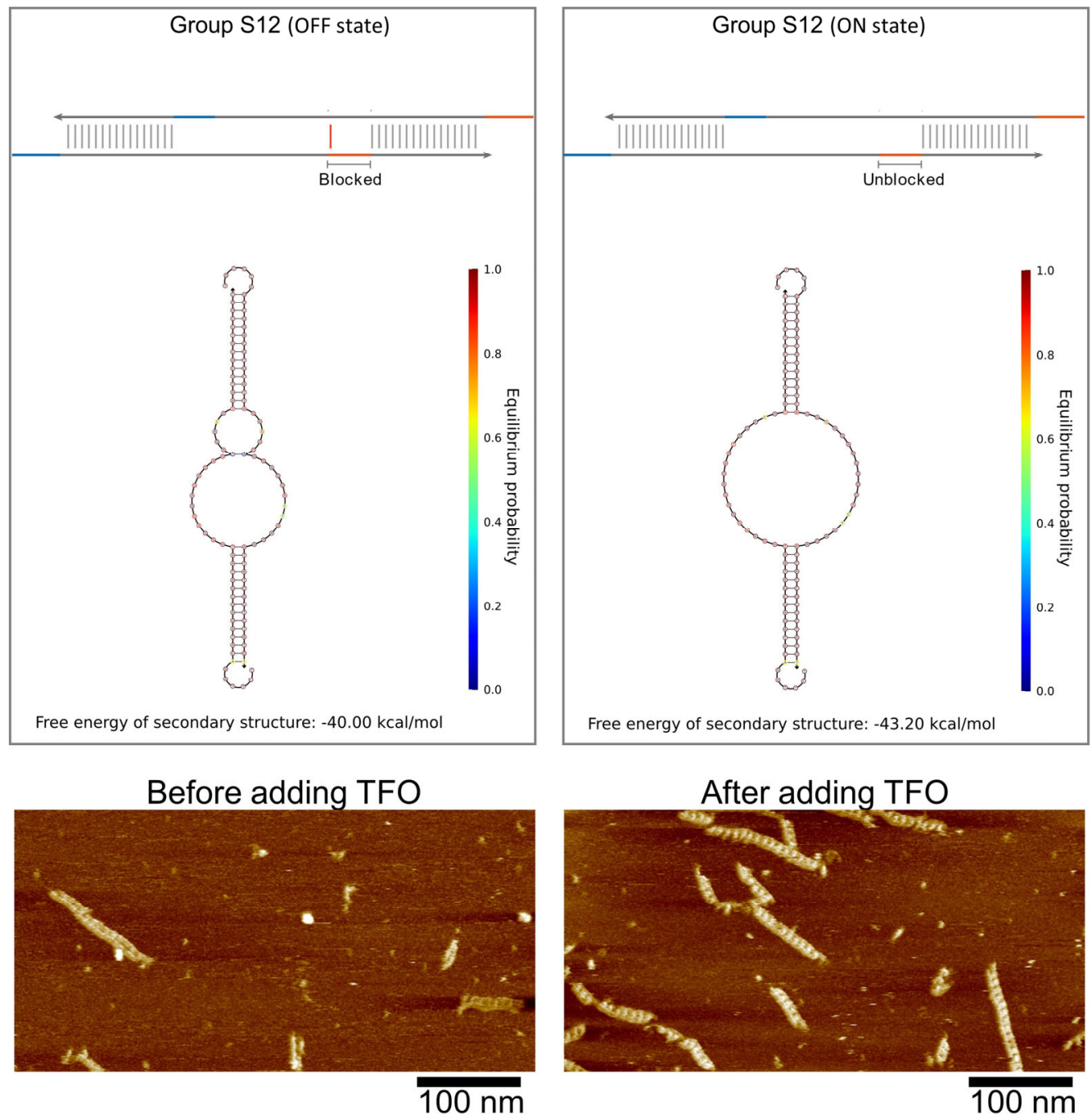

Figure S32. Schematic and simulated energetics parameter of the OFF state (left) and ON state (right) group S12 for one site allosteric transition screening (top panel). AFM images of group S12 before (left) and after (right) adding TFO modulator (bottom panel). Scale bars, $100 \mathrm{~nm}$. 

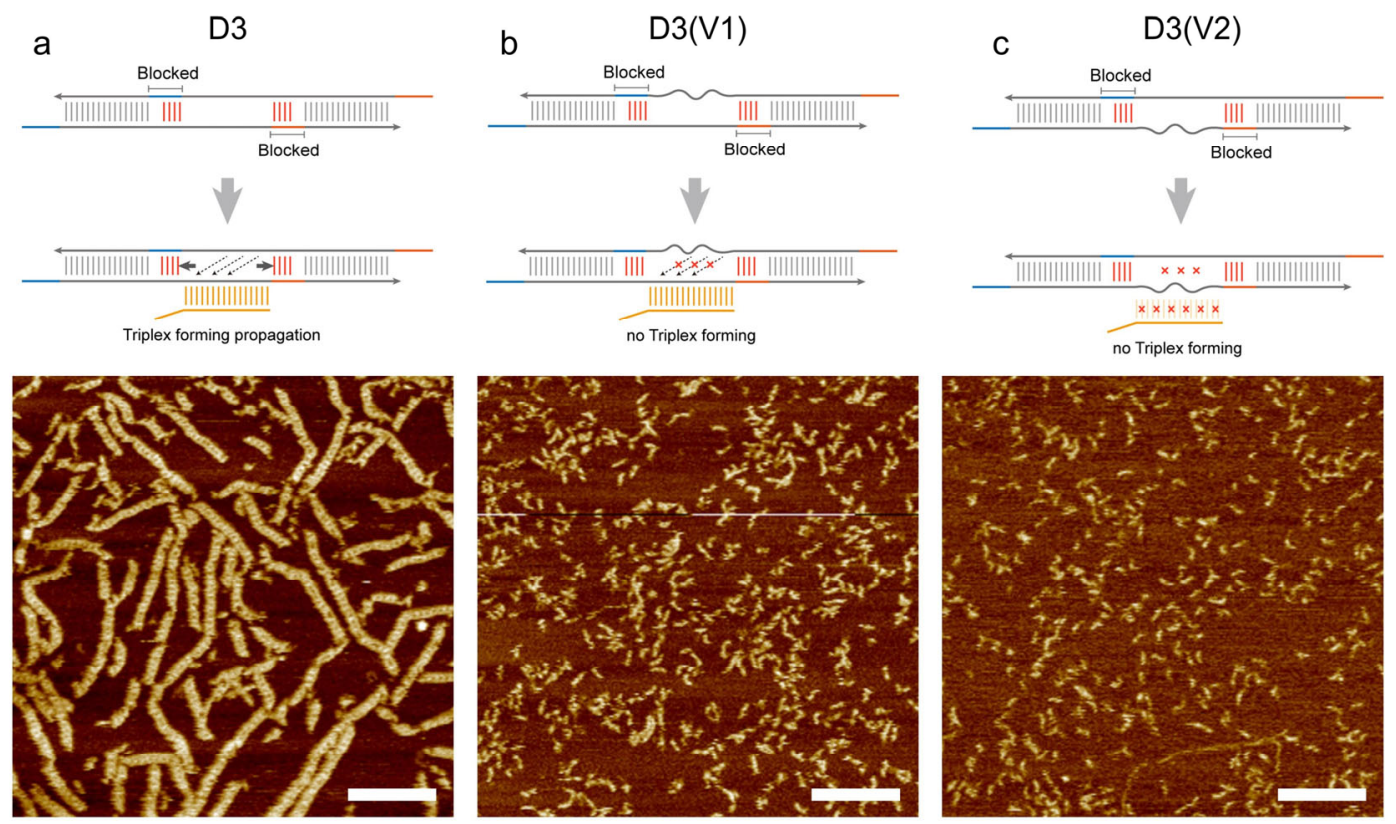

Figure S33. a, Schematic and AFM results of group D3 design after adding TFO modulator. b, Schematic and AFM results of control group D3(V1) design after adding TFO modulator. c, Schematic and AFM results of control group D3(V2) design after adding TFO modulator. Scale bars, $200 \mathrm{~nm}$. 

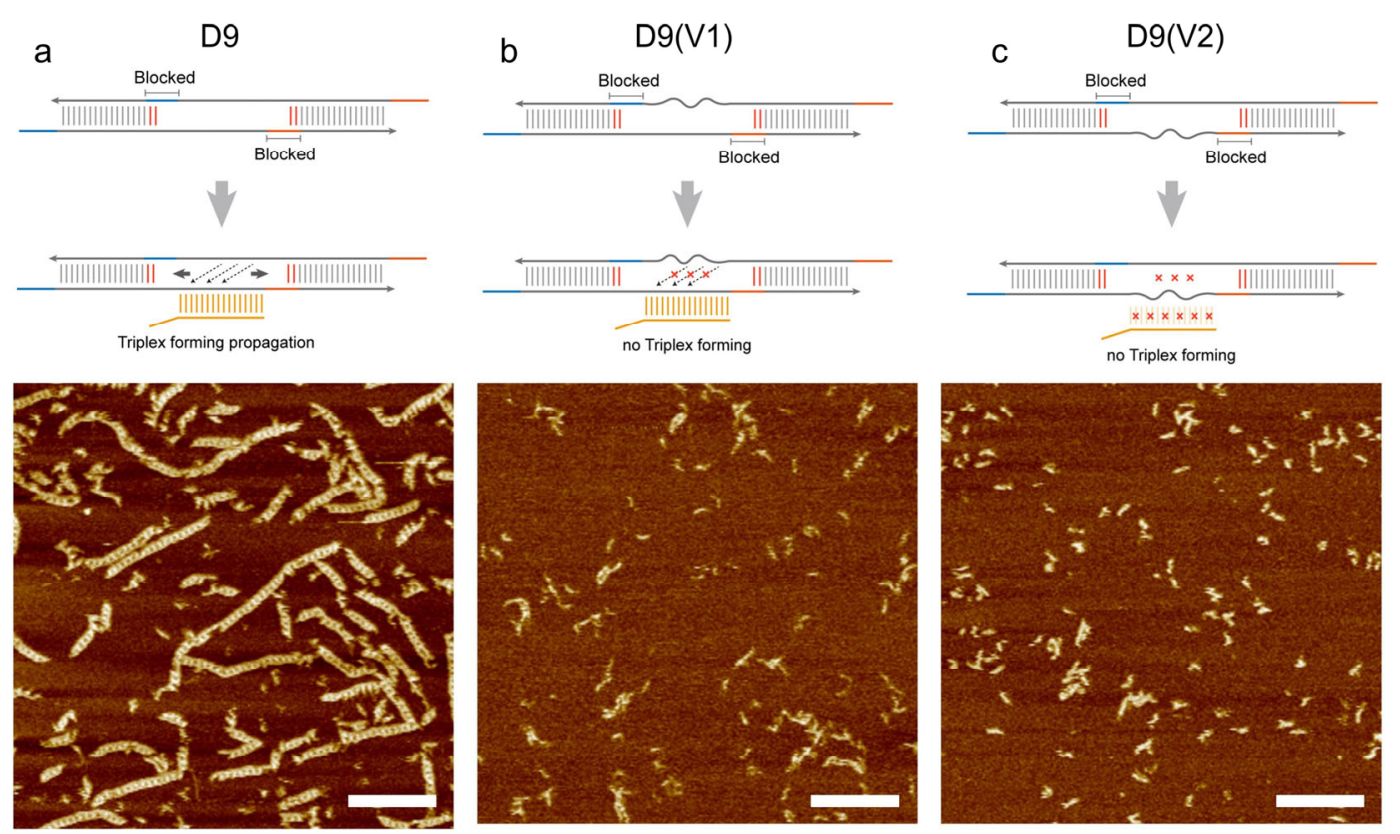

Figure S34. a, Schematic and AFM results of group D9 design after adding TFO modulator. b, Schematic and AFM results of control group D9(V1) design after adding TFO modulator. c, Schematic and AFM results of control group D9(V2) design after adding TFO modulator. Scale bars, $200 \mathrm{~nm}$. 
Normalized Fluorescence intensity value

\begin{tabular}{|c|c|c|c|c|c|}
\hline & Fluor. 1 & Fluor. 2 & Fluor. 3 & average & STD \\
\hline Buffer & 0.1039 & 0.1 & 0.102 & 0.102 & 0.0019 \\
\hline D3 & 0.6823 & 0.7179 & 0.7624 & 0.7209 & 0.0401 \\
\hline D9 & 0.7706 & 0.7747 & 0.7808 & 0.7754 & 0.0051 \\
\hline D3(V1) & 0.1272 & 0.1236 & 0.1265 & 0.1258 & 0.0019 \\
\hline D3(V2) & 0.1201 & 0.12 & 0.1243 & 0.1215 & 0.0025 \\
\hline D9(V1) & 0.1004 & 0.107 & 0.1119 & 0.1064 & 0.0058 \\
\hline D9(V2) & 0.0665 & 0.0704 & 0.0733 & 0.0701 & 0.0034 \\
\hline
\end{tabular}

Table S1. Fluorescence measurements of Thiazole Orange (TO)-triplex binding assay.

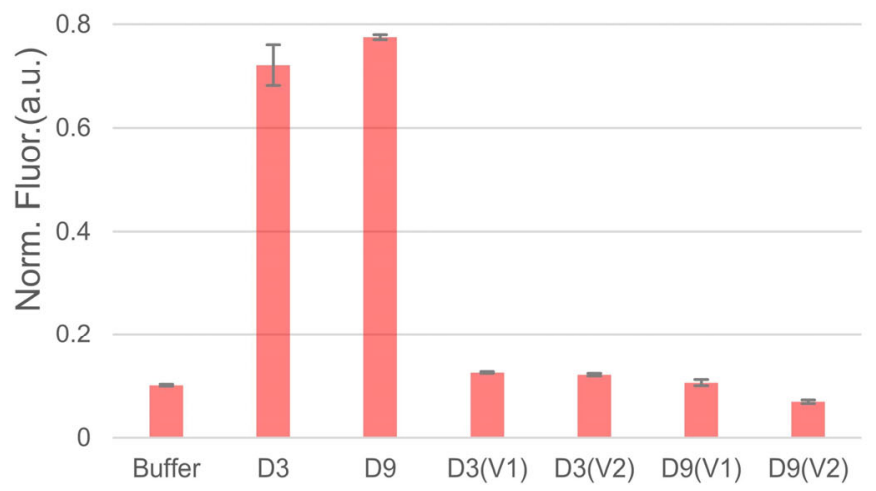

Figure S35. Histogram showing the normalized fluorescence intensity value of Thiazole Orange (TO)-triplex binding assay. 


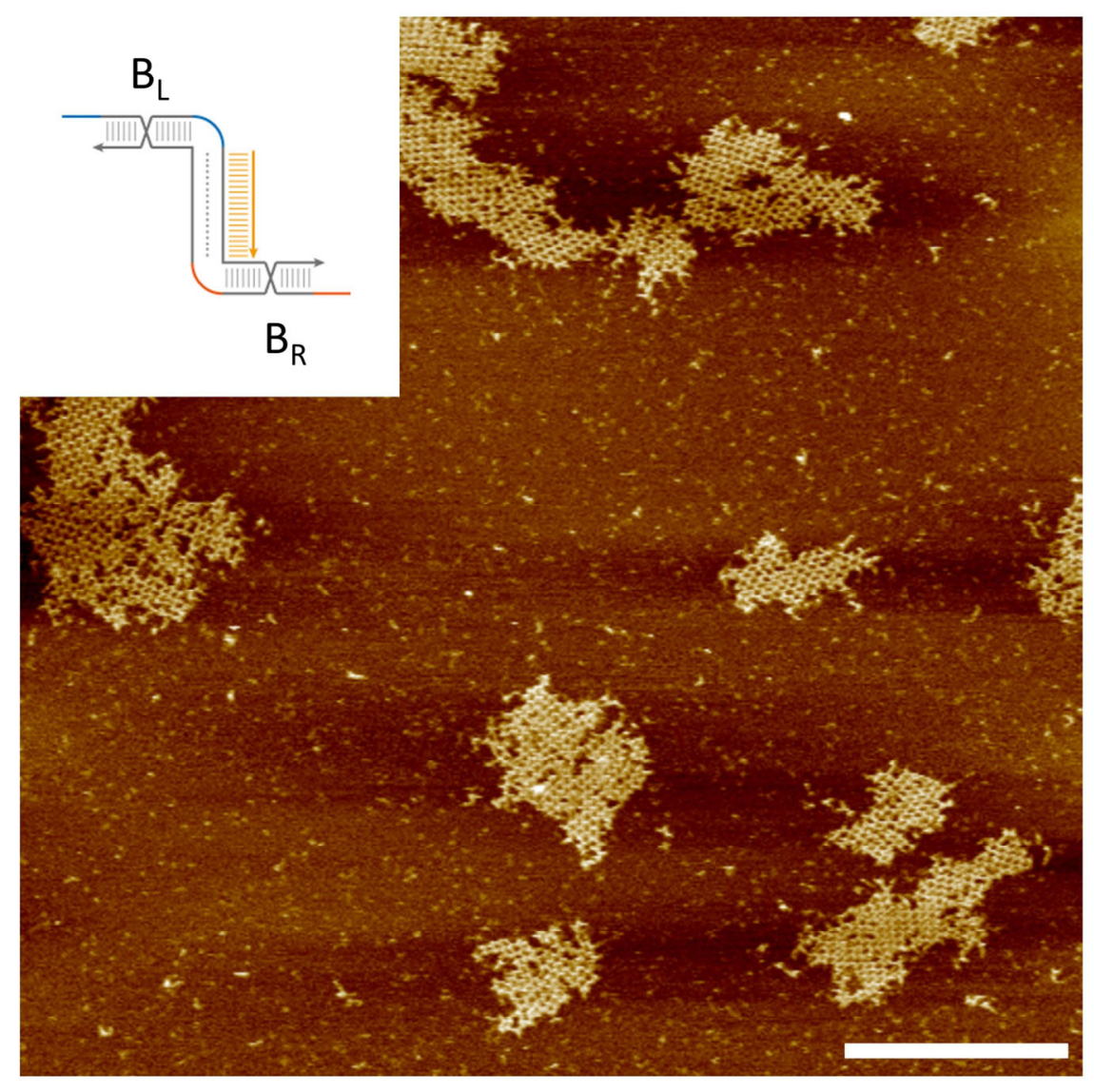

Figure S36. AFM result showing the $x$-form Z-switch than can assemble into $2 \mathrm{D}$ networks (branch L: 10 base pairs; triplex stem: 12 base triplets; branch R: 10 base pairs). Scale bar, $200 \mathrm{~nm}$. 


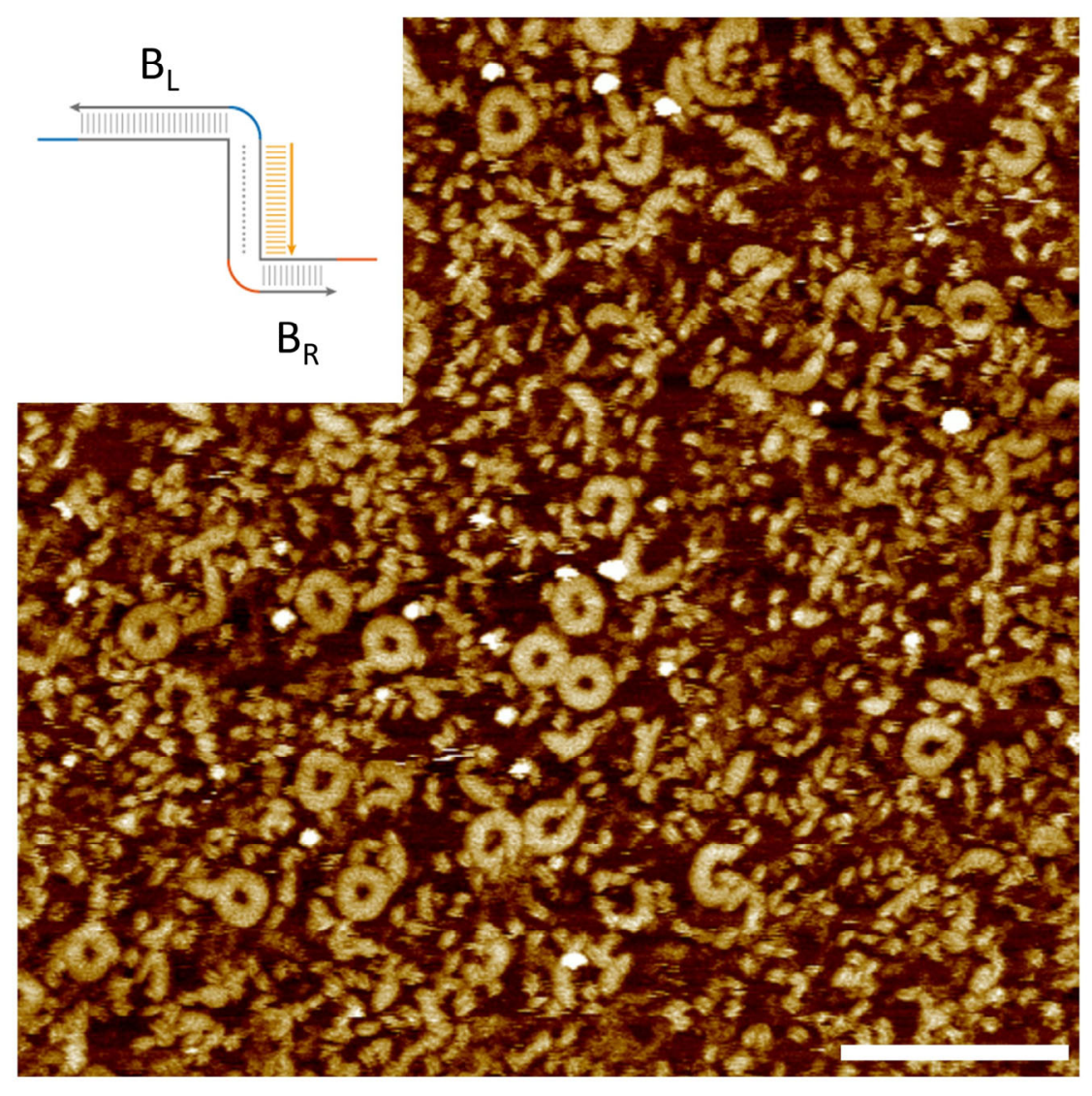

Figure S37. AFM result showing the $x$-form Z-switch than can assemble into 2D wheels (branch L: 16 base pairs; triplex stem: 24 base triplets; branch R: 6 base pairs). Scale bar, $200 \mathrm{~nm}$. 


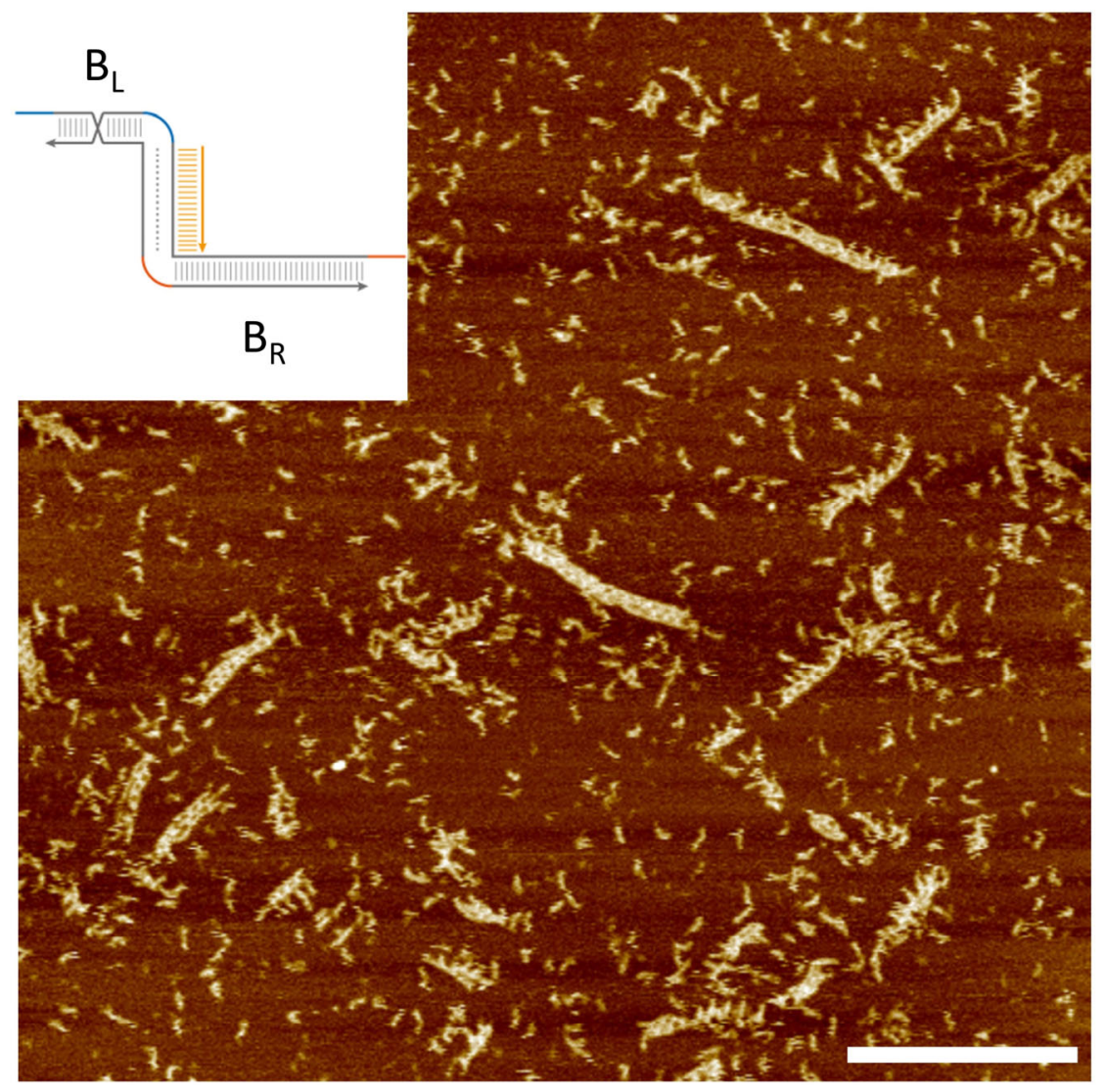

Figure S38. AFM result showing the $x$-form Z-switch than can assemble into 1D zippers (branch L: 10 base pairs; triplex stem: 12 base triplets; branch R: 26 base pairs). Scale bar, $200 \mathrm{~nm}$. 


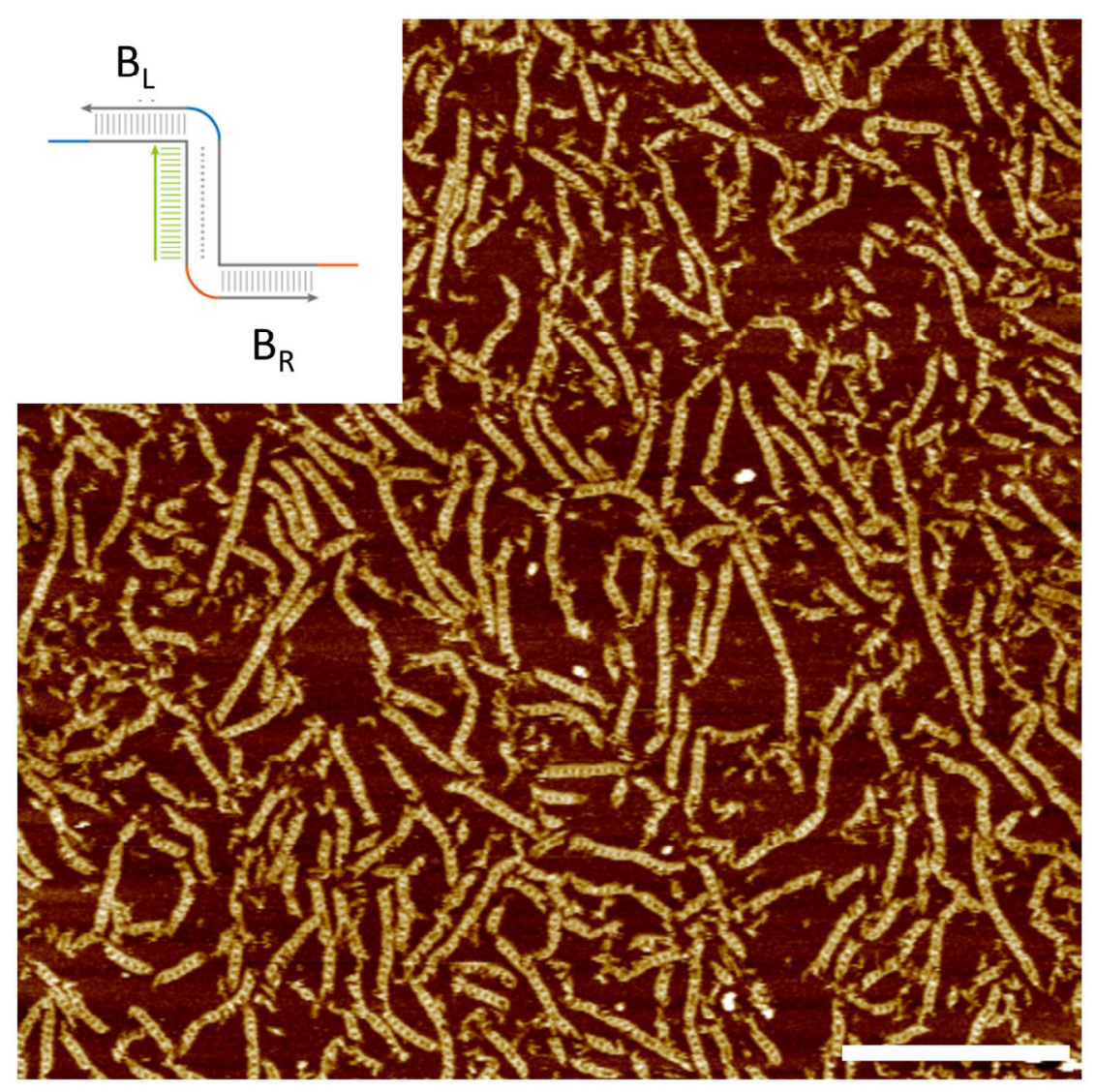

Figure S39. AFM result showing the $y$-form Z-switch than can assemble into 1D ladder arrays (branch L: 15 base pairs; triplex stem: 12 base triplets; branch R: 15 base pairs). Scale bar, $200 \mathrm{~nm}$. 


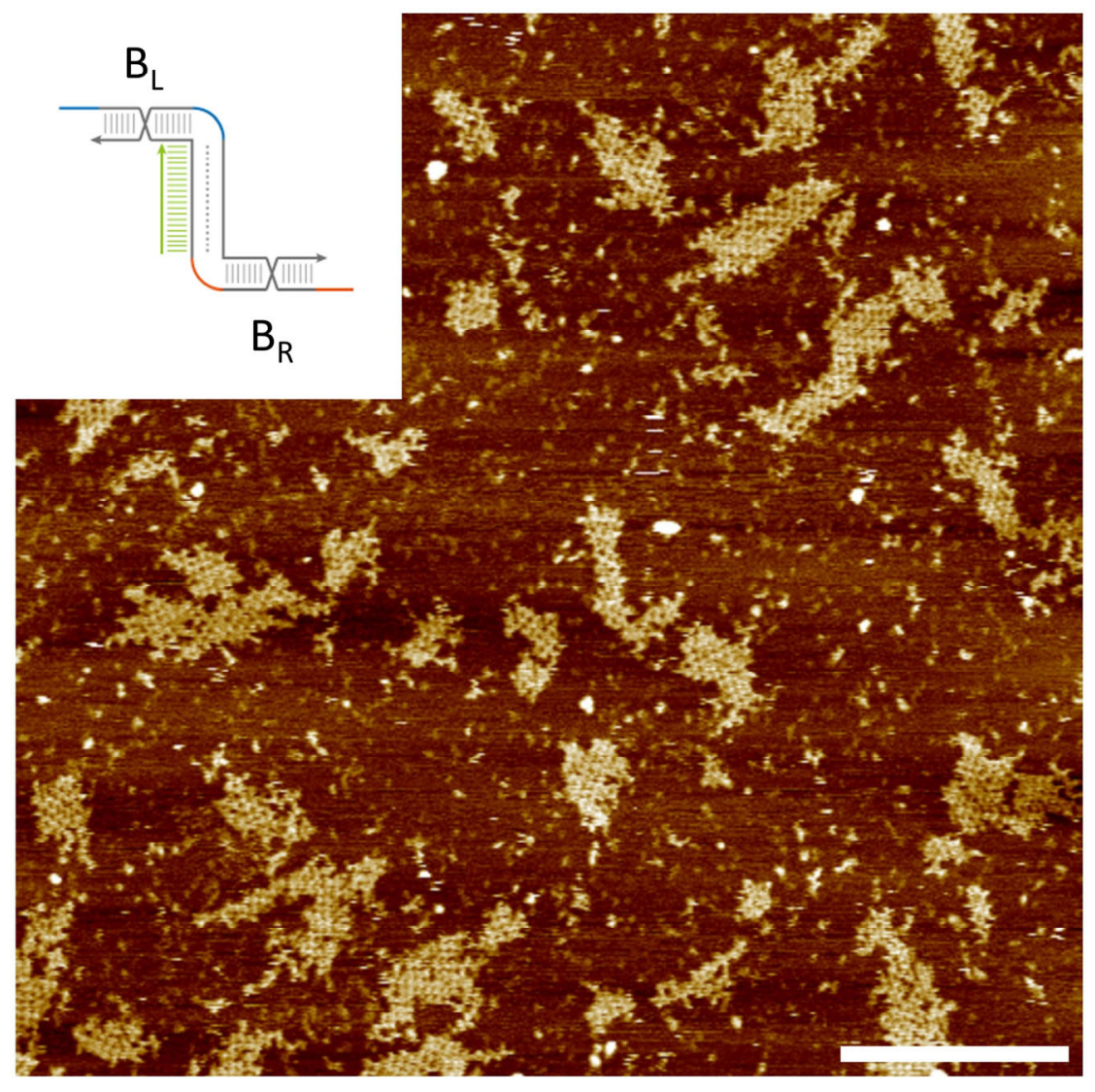

Figure S40. AFM result showing the $y$-form Z-switch than can assemble into $2 \mathrm{D}$ networks (branch L: 10 base pairs; triplex stem: 12 base triplets; branch R: 10 base pairs). Scale bar, $200 \mathrm{~nm}$. 


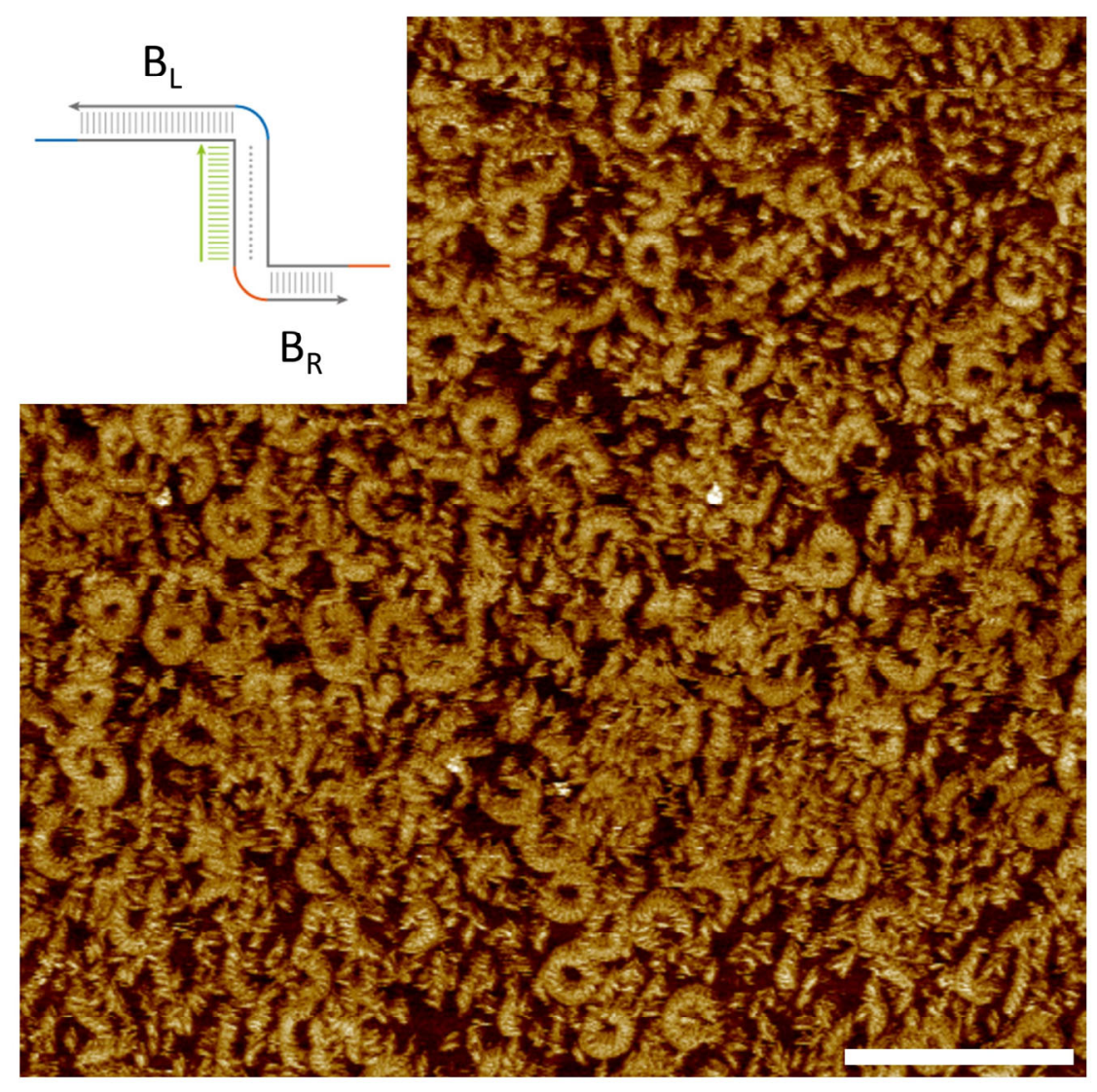

Figure S41. AFM result showing the $y$-form Z-switch than can assemble into 2D wheels (branch L: 16 base pairs; triplex stem: 24 base triplets; branch R: 6 base pairs). Scale bar, $200 \mathrm{~nm}$. 


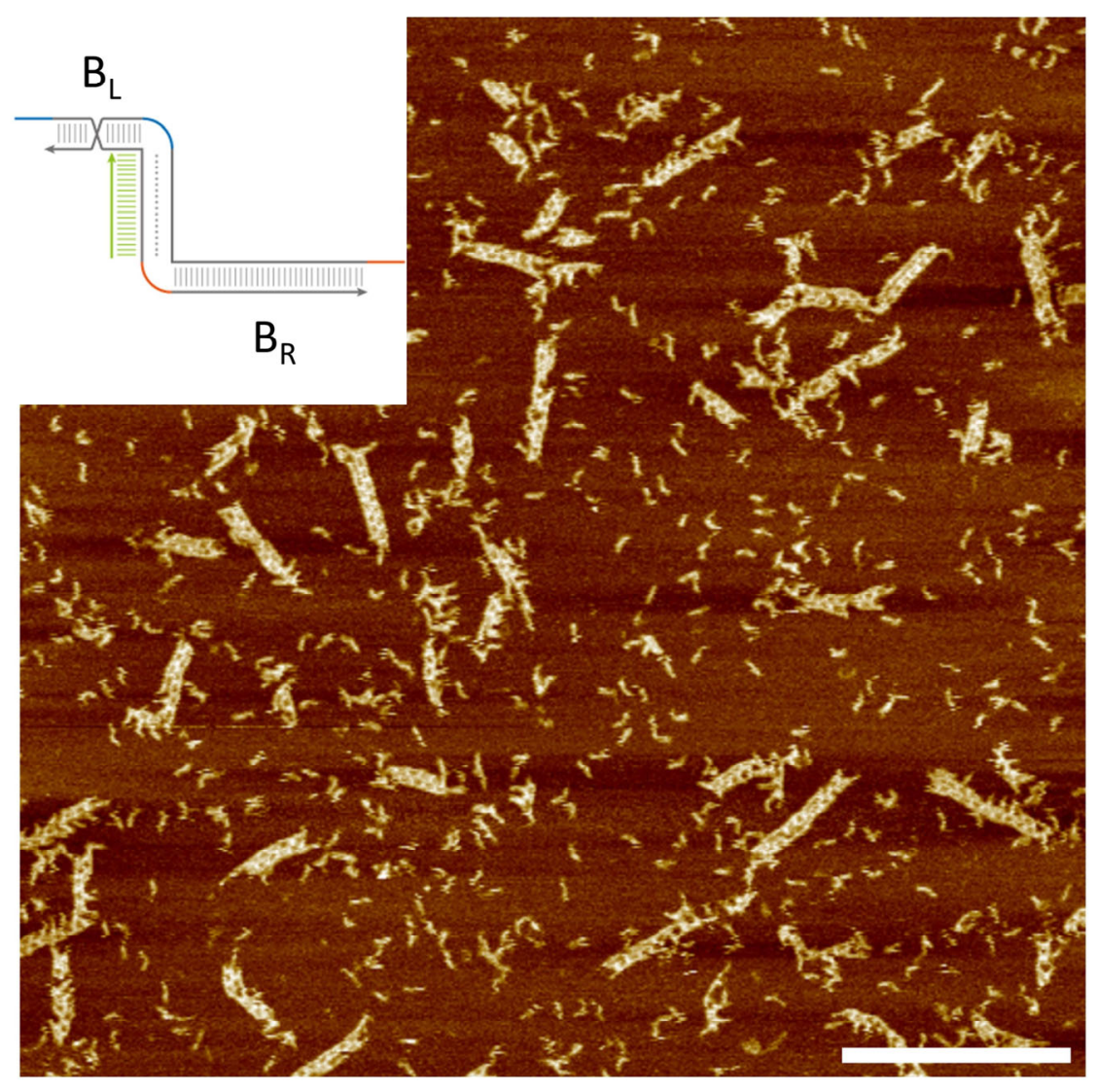

Figure S42. AFM result showing the $y$-form Z-switch than can assemble into 1D zippers (branch L: 10 base pairs; triplex stem: 12 base triplets; branch R: 26 base pairs). Scale bar, $200 \mathrm{~nm}$. 
a

$\stackrel{\substack{\text { Blocked } \\|||||||||||||||||||||||||||| \mid}}{\text { Blocked }}$

b

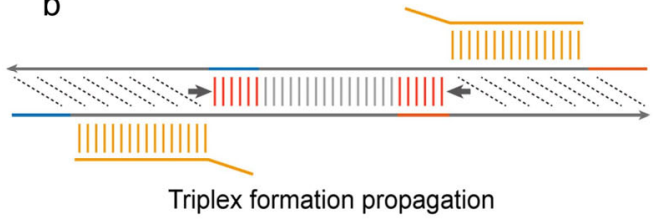

$d$

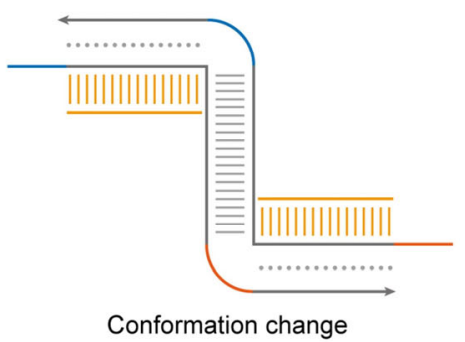

C

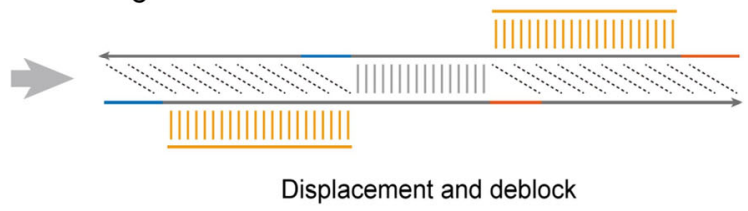

Figure S43. Allosteric transition of triplex-induced Z-switches for co-regulation. a, The blocked state of Z-switch. b, Triplex formation and propagation. c, Triplex propagation induces strand displacement and reopens the blocked site. d, The ON state after triplex-induced conformational change. 


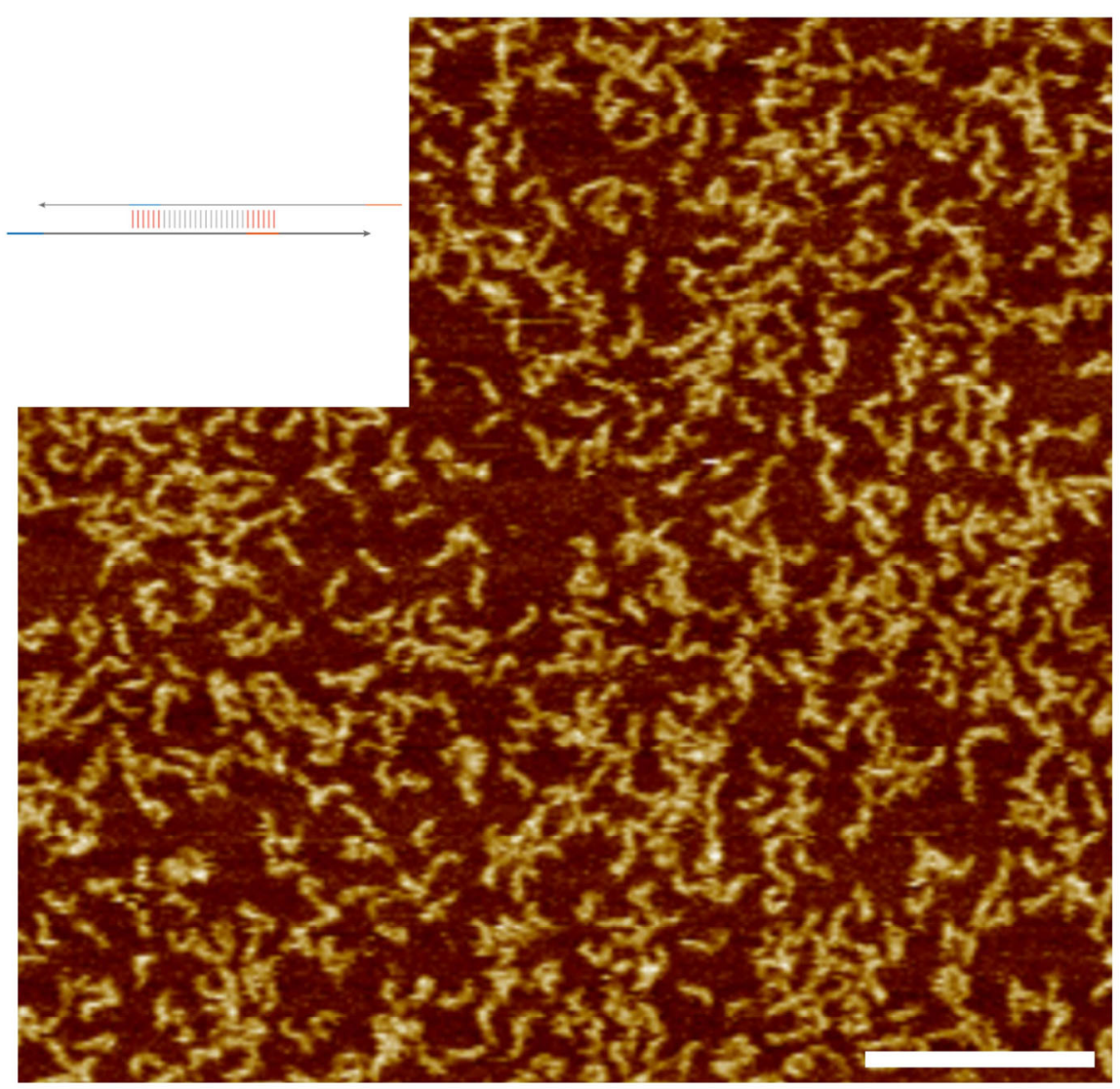

Figure S44. AFM result showing the blocked state of Z-switch for co-regulation before adding M1 and M2. Scale bar, $100 \mathrm{~nm}$. 


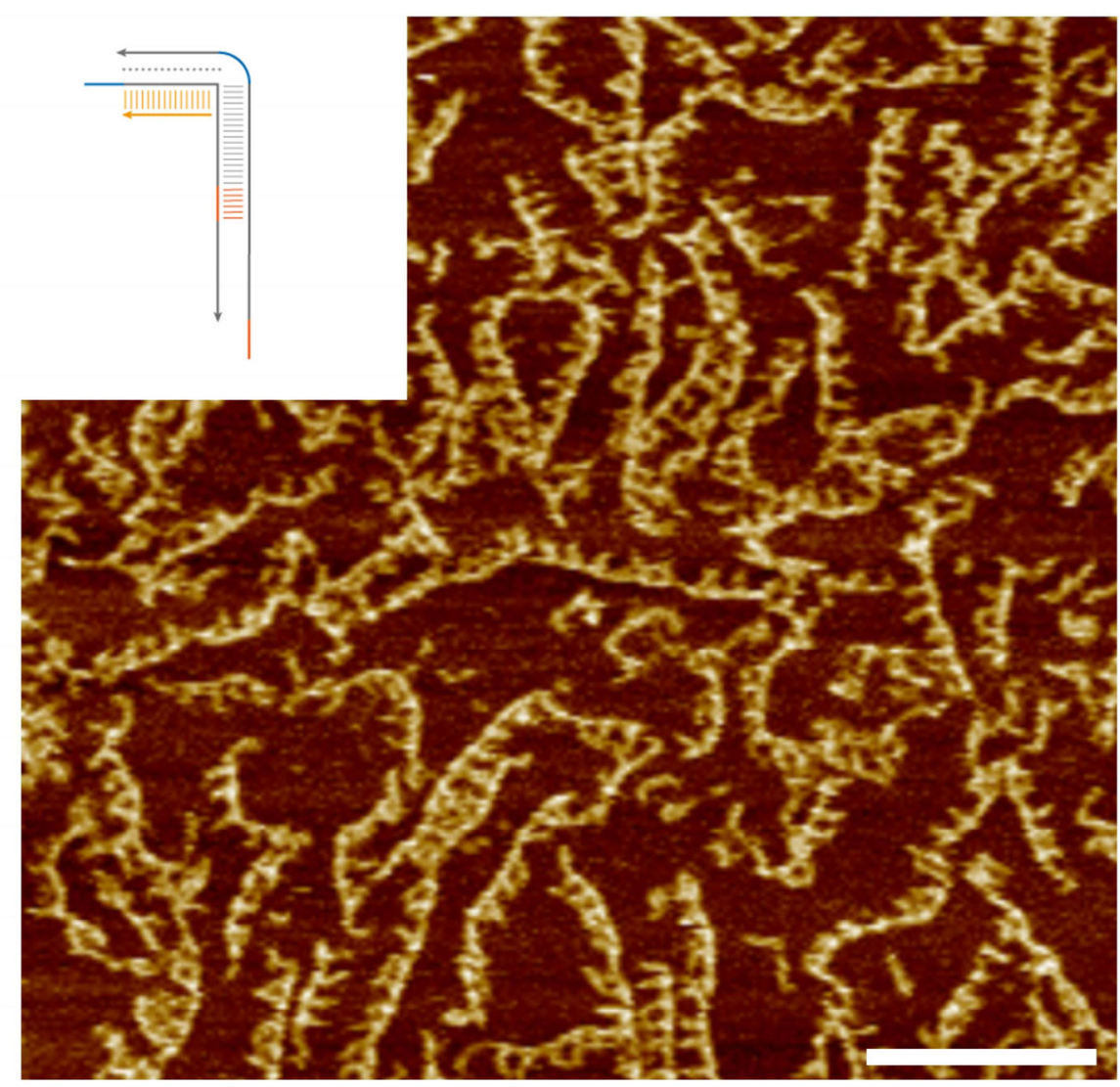

Figure S45. AFM result showing the blocked state of Z-switch for co-regulation after adding M1. Scale bar, $100 \mathrm{~nm}$. 


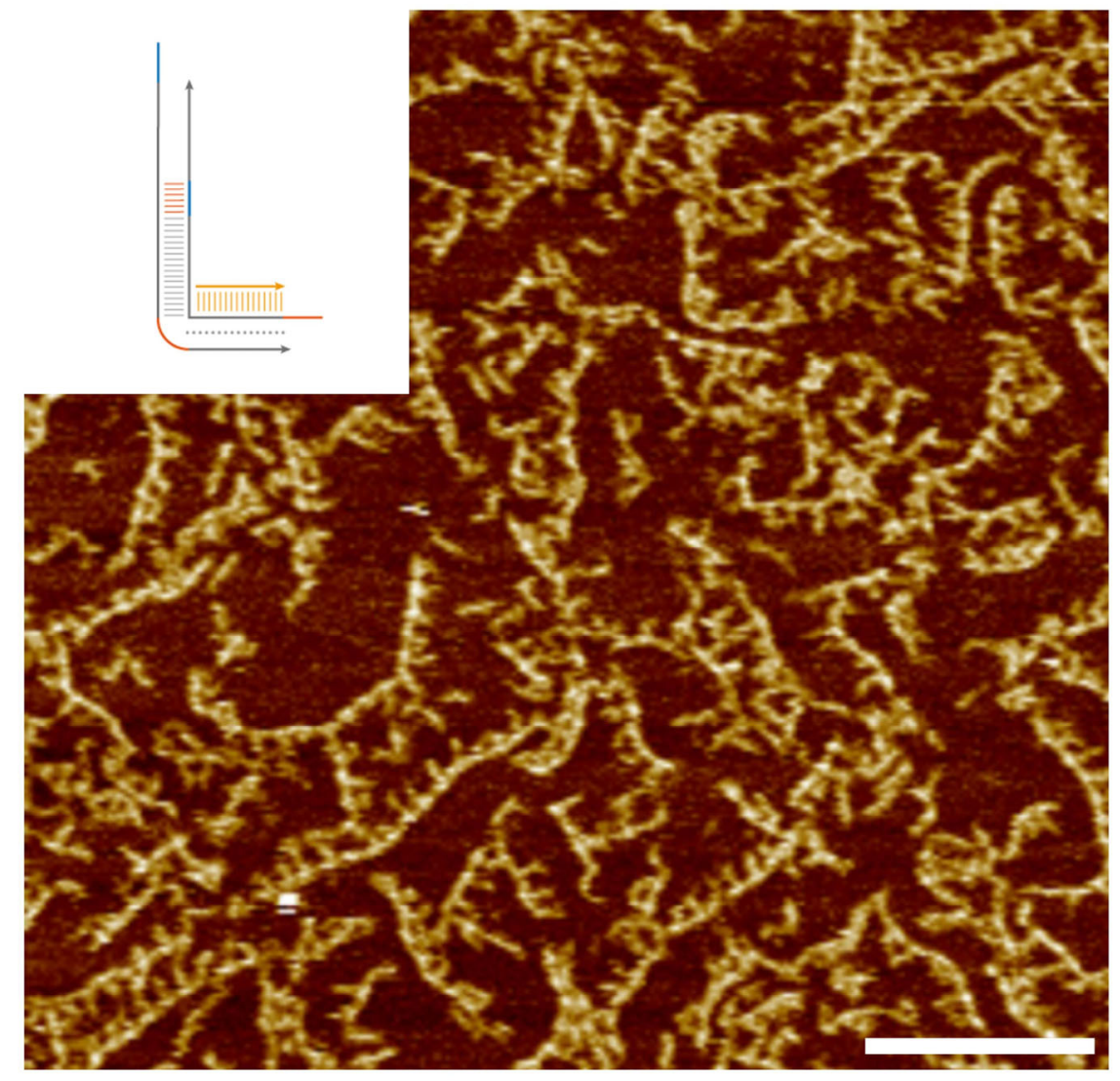

Figure S46. AFM result showing the blocked state of Z-switch for co-regulation after adding M2. Scale bar, $100 \mathrm{~nm}$. 


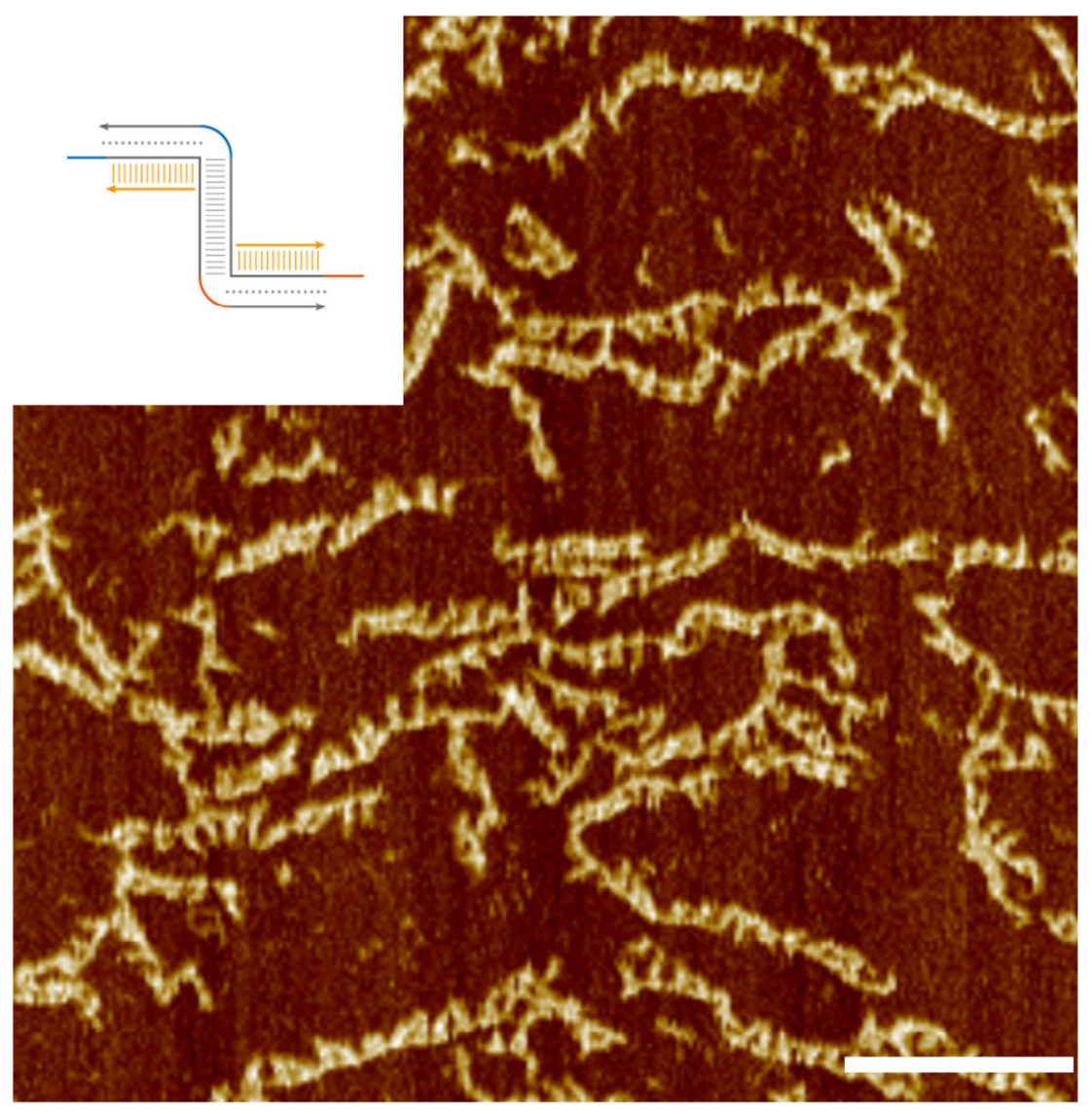

Figure S47. AFM result showing the blocked state of Z-switch for co-regulation after adding M1 \& M2. Scale bar, $100 \mathrm{~nm}$. 


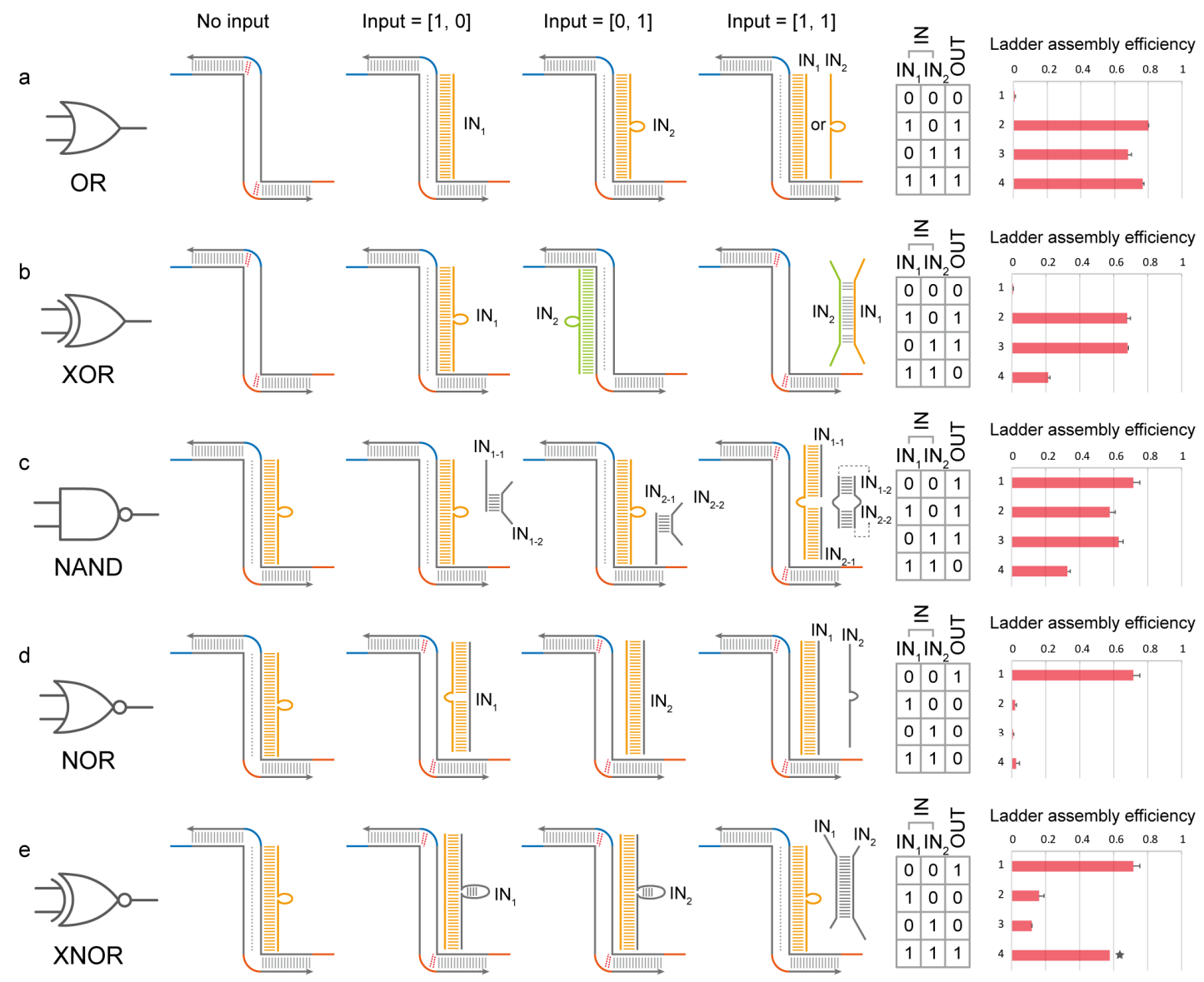

Figure S48. Boolean logic operations based on allosteric nanodevices. a-e, The five logic gates were displayed in blocked or open state without inputs, with $\mathrm{IN}_{1}, \mathrm{IN}_{2}$ or both, according to the rules specified by each logic gate. The predicted output values and allosteric assembly efficiency are displayed to the right. a, OR gate. b, XOR gate. c, NAND gate. d, NOR gate. e, XNOR gate. Bars in red: assembly efficiency of ladder array. Asterisk indicates the missing of error bar. See methods part and Table S4 for data collection and processing details. 

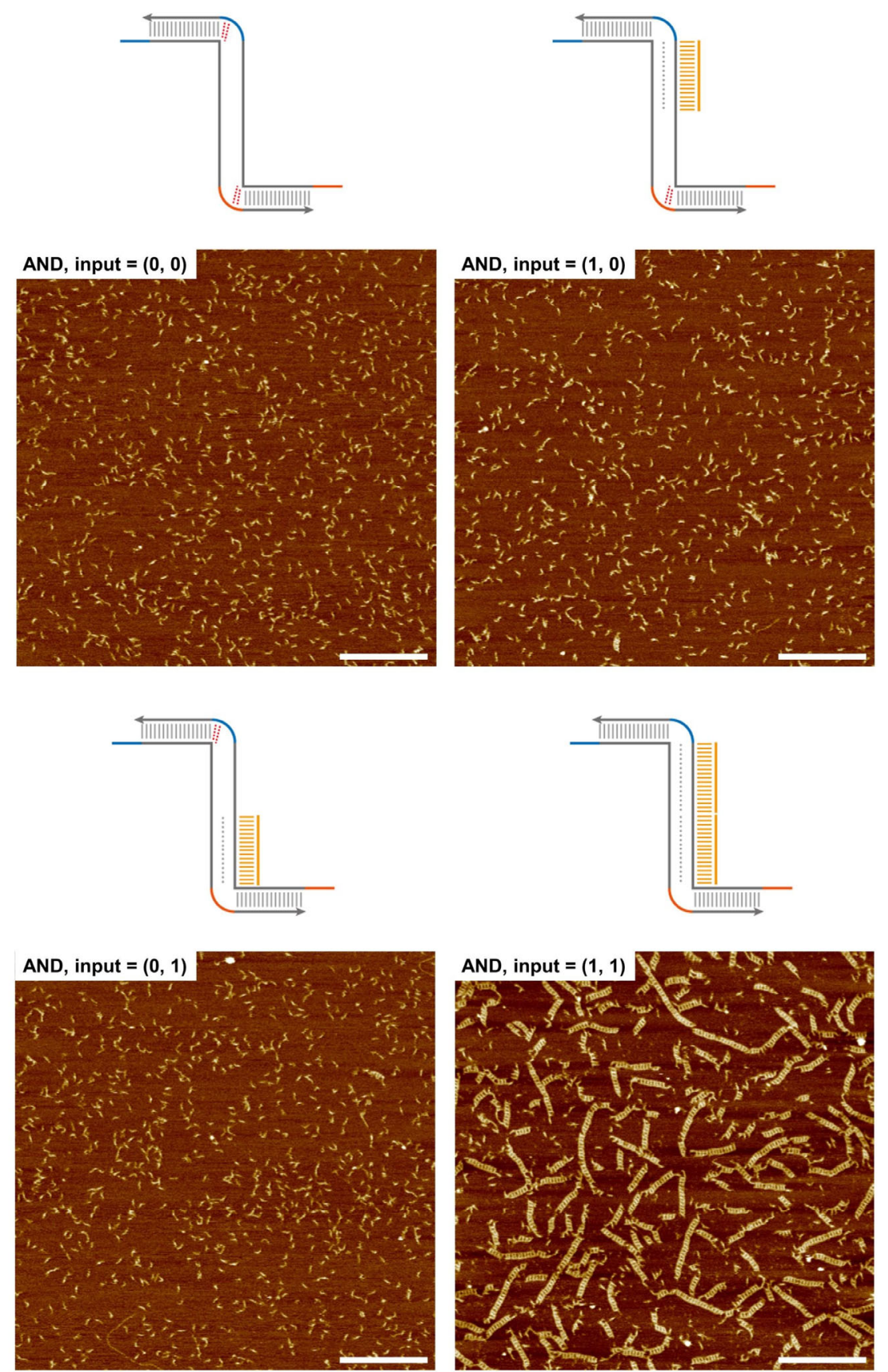

Figure S49. AFM results showing the AND logic gates with inputs $(0,0),(1,0),(0,1)$ and $(1,1)$. Scale bars, $200 \mathrm{~nm}$. 


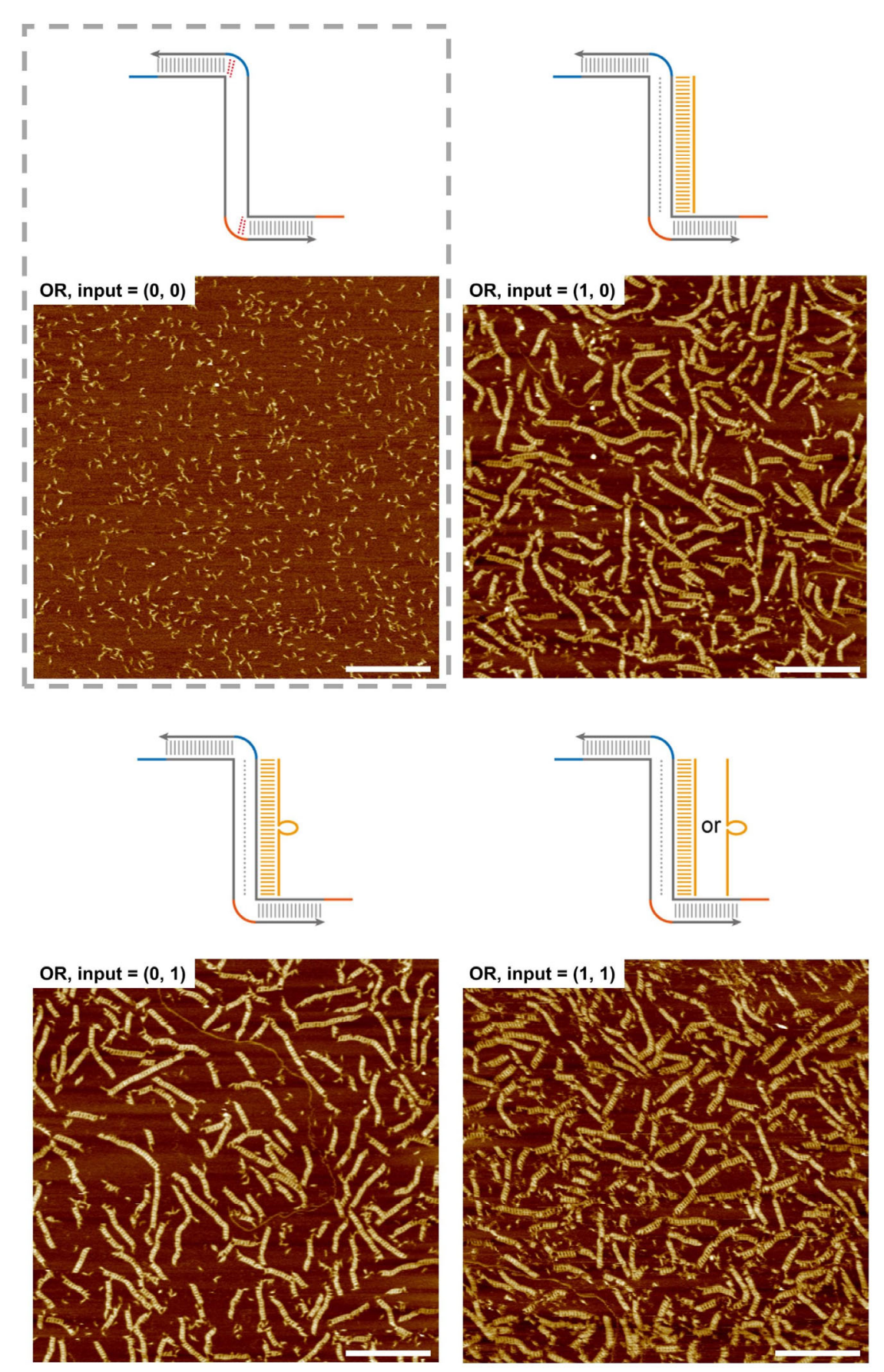

Figure S50. AFM results showing the OR logic gates with inputs $(1,0),(0,1)$ and $(1$, $1)$. The input $(0,0)$ of OR gate is the same as input $(0,0)$ of AND gate due to the same sequence design. Scale bars, $200 \mathrm{~nm}$. 

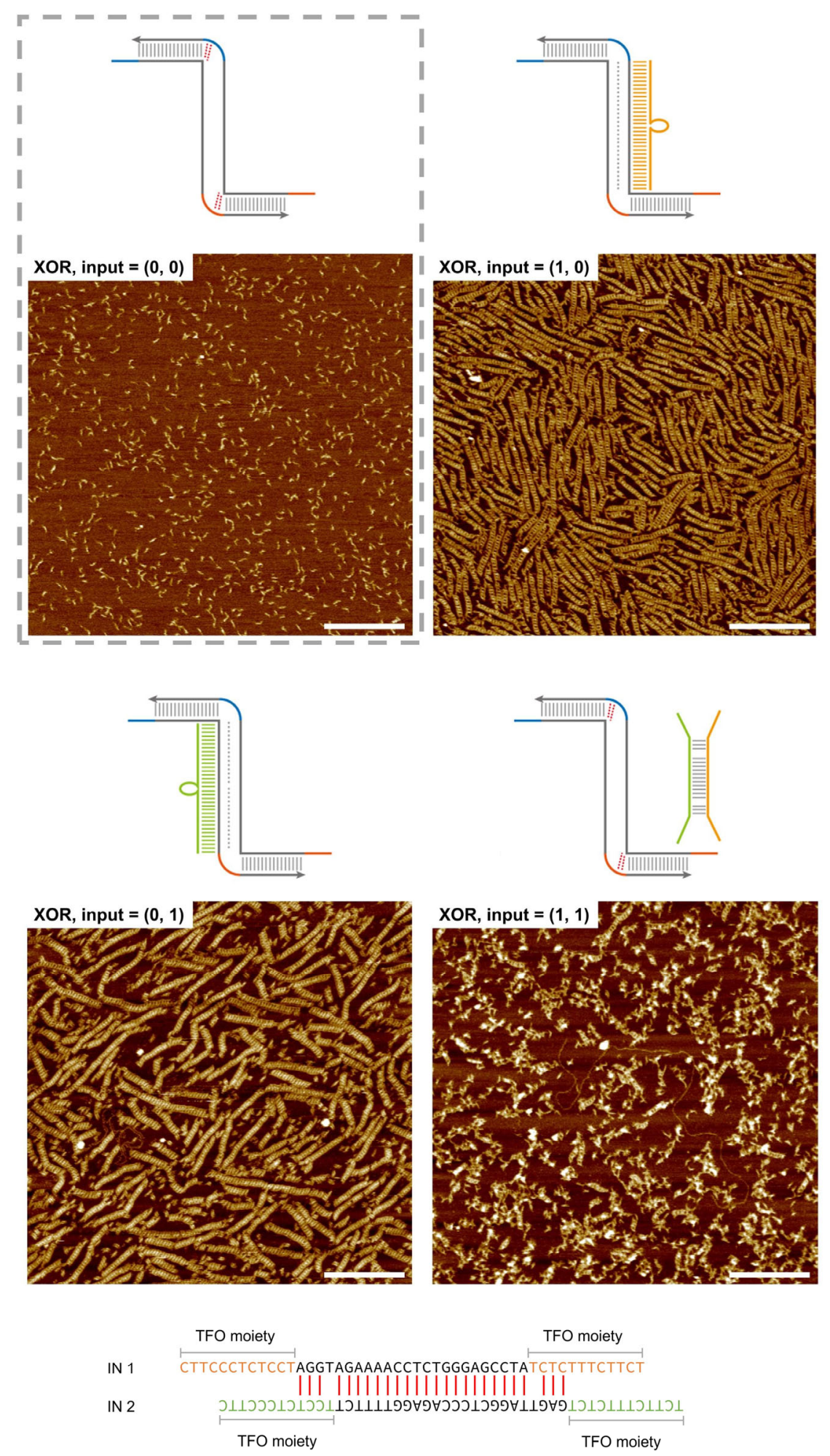

Figure S51. AFM results showing the XOR logic gates with inputs $(1,0),(0,1)$ and $(1$, $1)$. The input $(0,0)$ of XOR gate is the same as input $(0,0)$ of AND gate due to the same sequence design. Scale bars, $200 \mathrm{~nm}$. Sequences of IN-1 and IN-2 are displayed to show the strand pairing principles. 

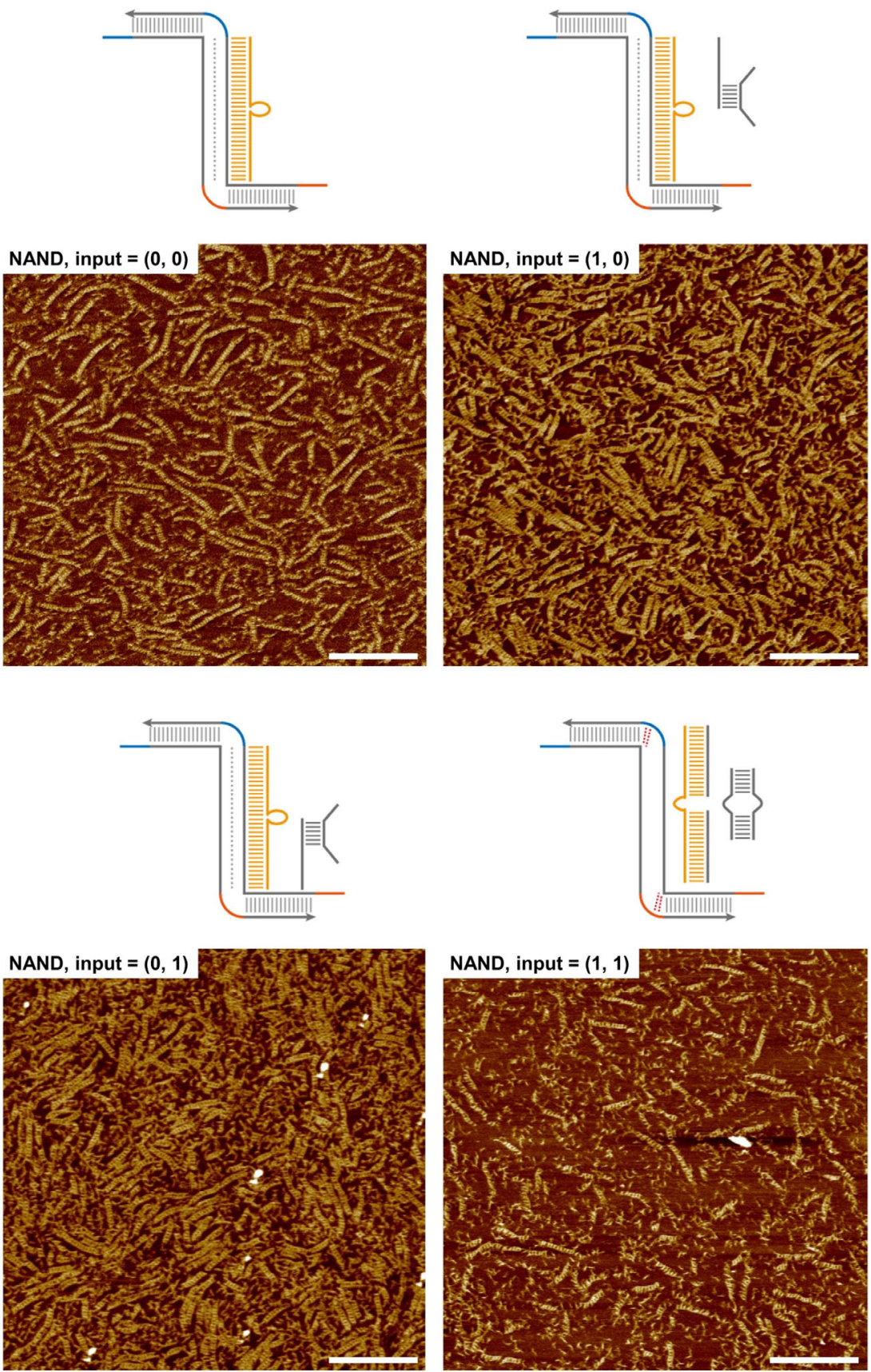

NAND, input $=(1,1)$
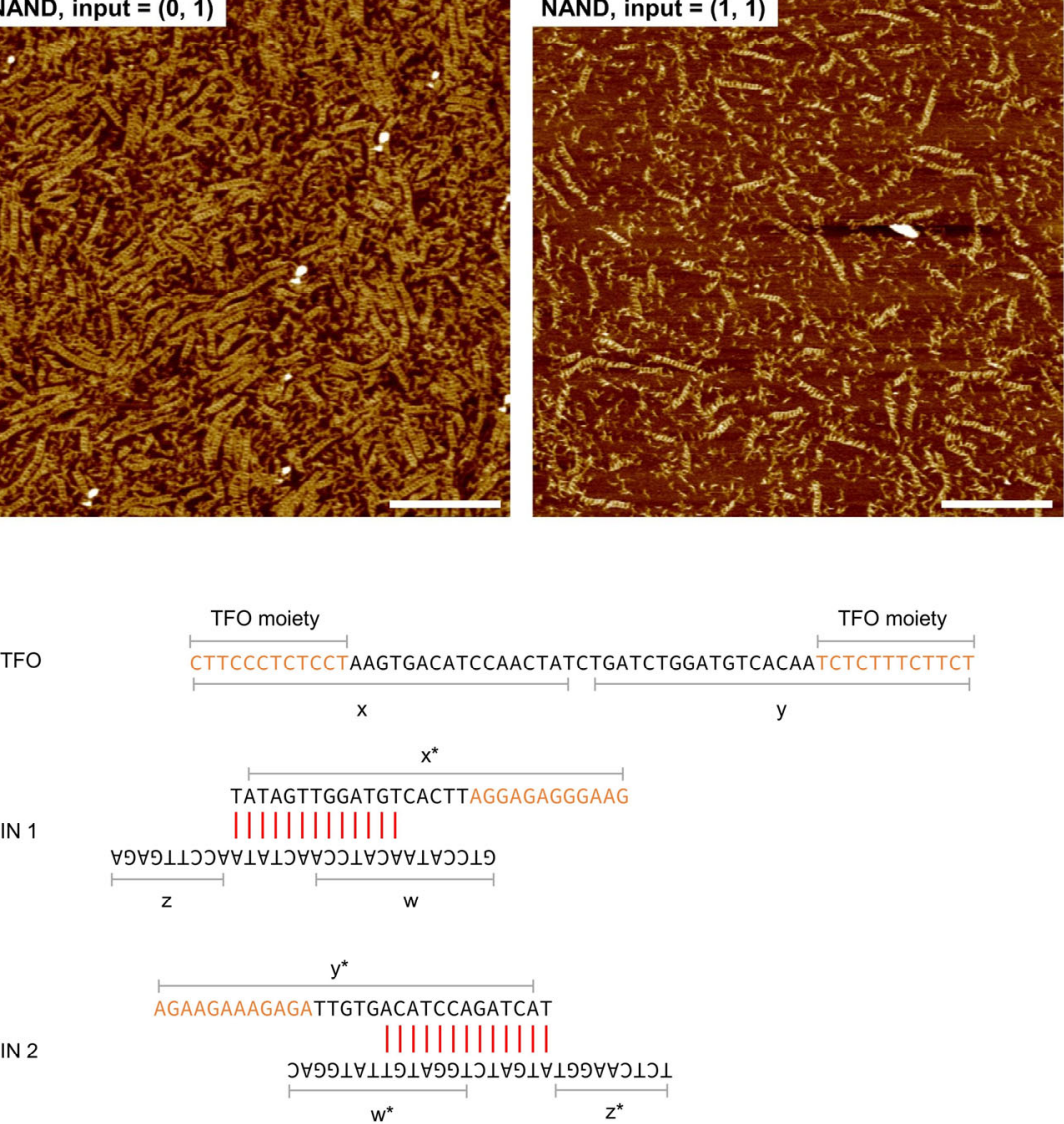
Figure S52. AFM results showing the NAND logic gates with inputs $(0,0),(1,0),(0$, $1)$ and $(1,1)$. Scale bars, $200 \mathrm{~nm}$. Sequences of the TFO, IN-1 and IN-2 are displayed to show the strand pairing principles (the letter and its asterisked form represent complementary sequences).

To implement NAND logic gate - a representative of the second regulation type, inside the TFO modulators we placed a toehold region including two toehold sequences, each targeted by one of the two inputs (each input is composed of a displacement strand and its inhibitor strand). Due to the malfunction of the displacement strand (which is occupied by its inhibitor strand), any single input (input $=[1,0],[0,1]$ ) cannot fully displace the half of the TFO modulators (displacement ratio below $\sim 40 \%$ ) thus most ladder arrays kept unchanged (output $=1)$. Once both inputs were added (input $=[1,1]$ ) the two displacement strands were released (two inhibitor strands formed a more stable structure), and strand displacement on TFO modulator (displacement ratio $\sim 70 \%$ ) took place then the ladder array gradually dissociated (output $=0$ ). 


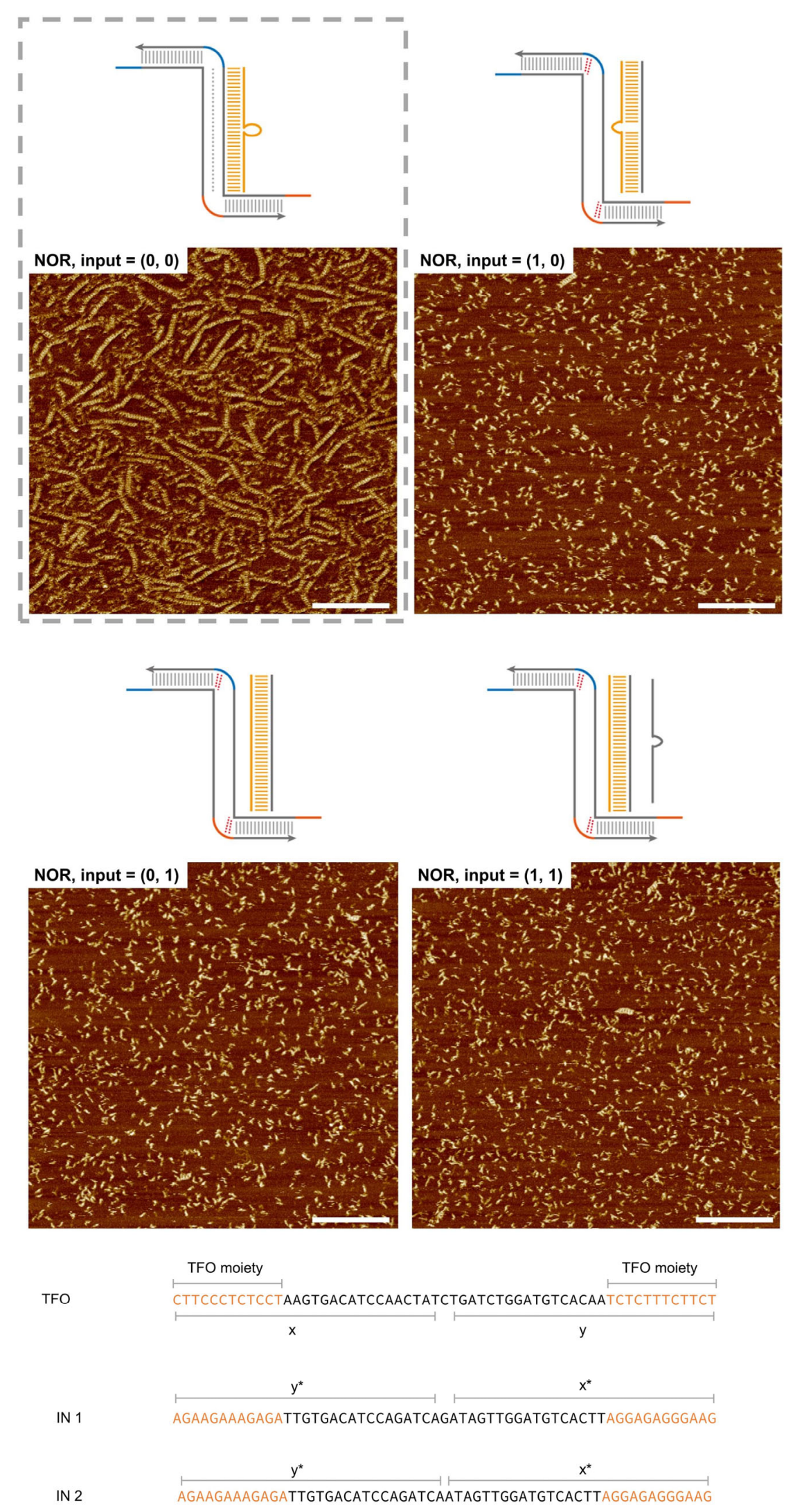

Figure S53. AFM results showing the NOR logic gates with inputs $(1,0),(0,1)$ and $(1$, $1)$. The input $(0,0)$ of NOR gate is the same as input $(0,0)$ of NAND gate due to the same sequence design. Scale bars, $200 \mathrm{~nm}$. Sequences of the TFO, IN-1 and IN-2 are 
displayed to show the strand pairing principles (the letter and its asterisked form represent complementary sequences). 

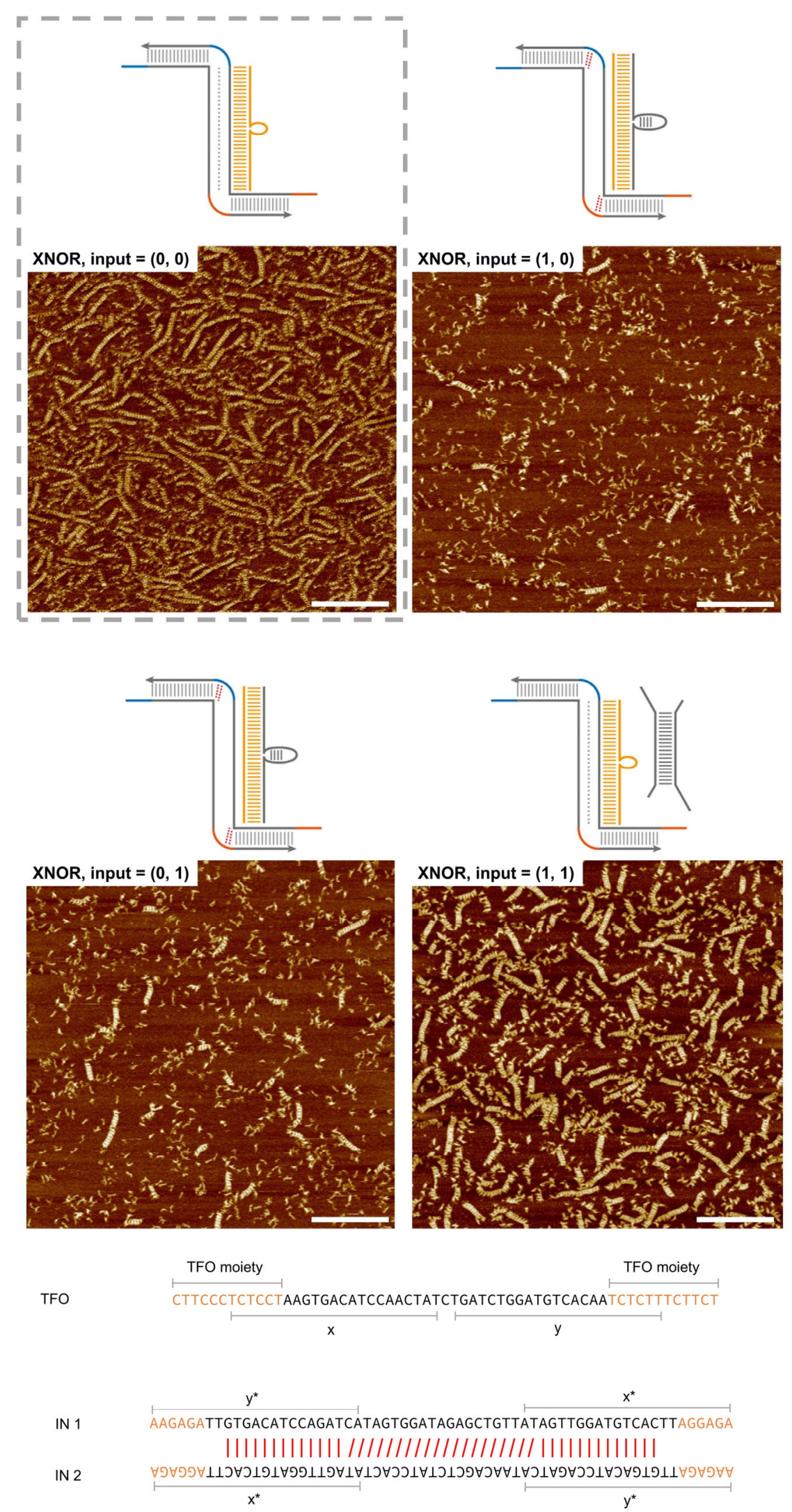

Figure S54. AFM results showing the XNOR logic gates with inputs $(1,0),(0,1)$ and $(1,1)$. The input $(0,0)$ of XNOR gate is the same as input $(0,0)$ of NAND gate due to the same sequence design. Scale bars, $200 \mathrm{~nm}$. Sequences of the TFO, IN-1 and IN-2 
are displayed to show the strand pairing principles (the letter and its asterisked form represent complementary sequences). 

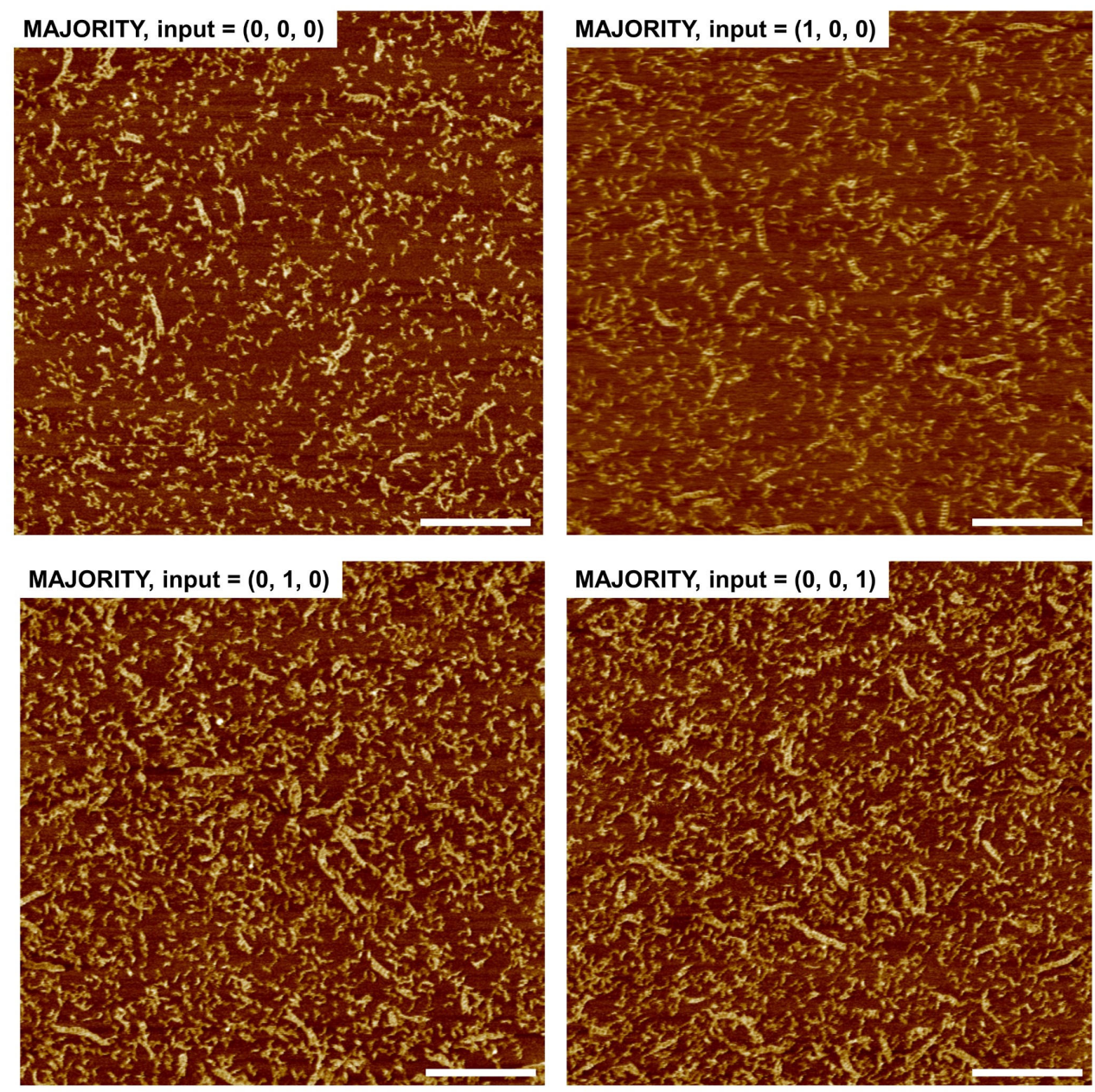

Figure S55. AFM results showing the MAJORITY function with inputs $(0,0,0),(1,0$, $0),(0,1,0)$ and $(0,0,1)$. Scale bars, $200 \mathrm{~nm}$. 

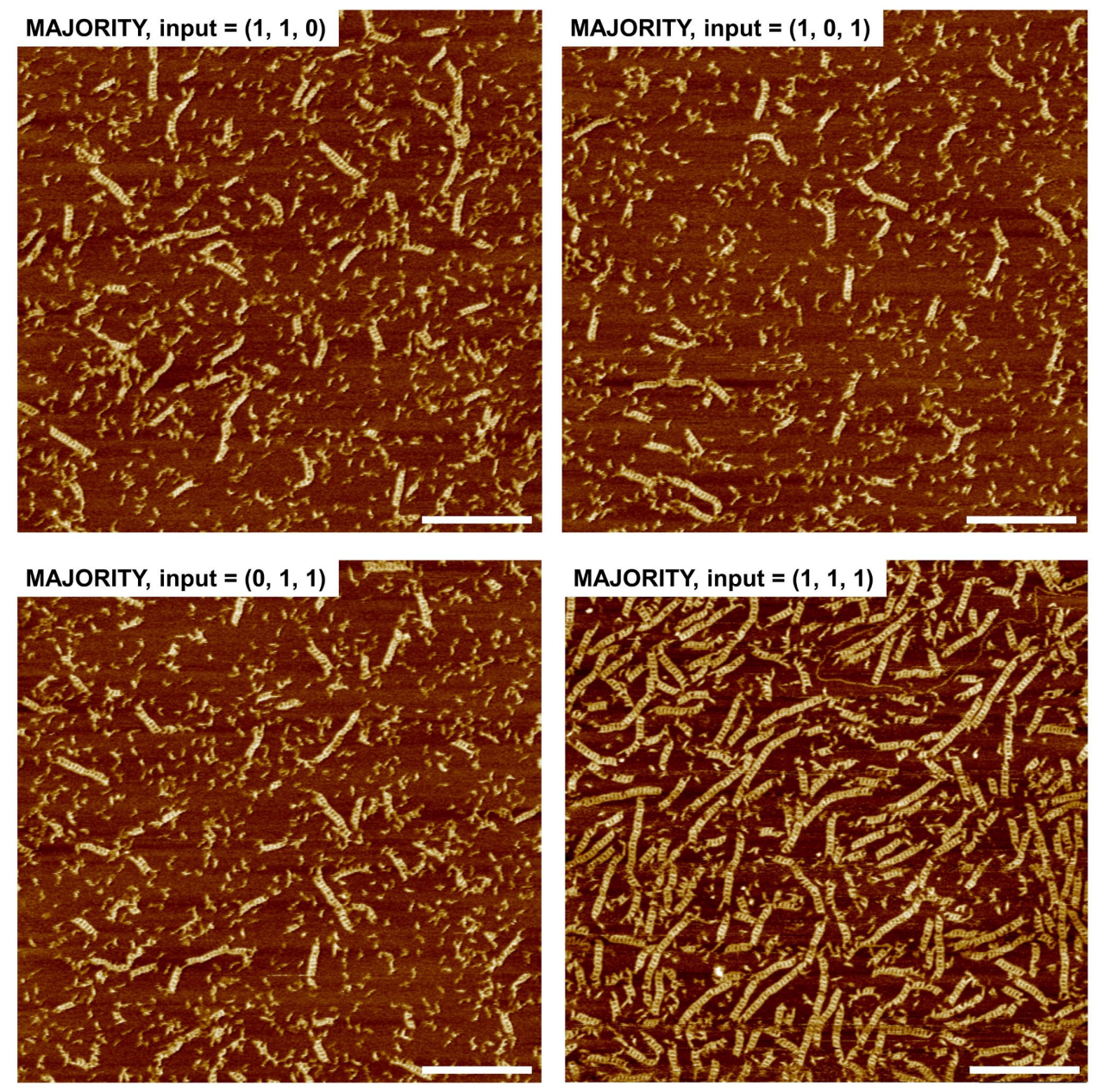

Figure S56. AFM results showing the MAJORITY function with inputs $(1,1,0),(1,0$, $1),(0,1,1)$ and $(1,1,1)$. Scale bars, $200 \mathrm{~nm}$. 


\begin{tabular}{|c|c|c|c|c|c|c|c|c|c|c|c|c|}
\hline \multicolumn{13}{|c|}{ Table S2. Transition ratio of 2 sites screening } \\
\hline & D1 & $\mathrm{D} 2$ & D3 & D4 & D5 & D6 & D7 & D8 & D9 & D10 & D11 & D12 \\
\hline before & $757 / 769$ & $150 / 286$ & $2 / 166$ & $0 / 176$ & $0 / 129$ & $6 / 223$ & $0 / 132$ & $2 / 181$ & $0 / 124$ & $192 / 504$ & $314 / 496$ & $355 / 447$ \\
\hline ratio & $98.4 \%$ & $52.4 \%$ & $1.2 \%$ & $0.0 \%$ & $0.0 \%$ & $2.7 \%$ & $0.0 \%$ & $1.1 \%$ & $0.0 \%$ & $38.1 \%$ & $63.3 \%$ & $79.4 \%$ \\
\hline after & $488 / 505$ & $152 / 348$ & $80 / 176$ & 0/191 & $0 / 79$ & $0 / 170$ & $2 / 155$ & 0/104 & $121 / 254$ & $304 / 455$ & $61 / 108$ & $40 / 70$ \\
\hline ratio & $96.6 \%$ & $43.7 \%$ & $45.6 \%$ & $0.0 \%$ & $0.0 \%$ & $0.0 \%$ & $1.3 \%$ & $0.0 \%$ & $47.6 \%$ & $66.8 \%$ & $56.5 \%$ & $57.1 \%$ \\
\hline
\end{tabular}

\begin{tabular}{|c|c|c|c|c|c|c|c|c|c|c|c|c|}
\hline \multicolumn{13}{|c|}{ Table S3. Transition ratio of 1 site screening } \\
\hline & S1 & S2 & S3 & S4 & S5 & S6 & S7 & S8 & S9 & $\mathrm{S} 10$ & $\mathrm{~S} 11$ & $\mathrm{~S} 12$ \\
\hline before & $757 / 769$ & $180 / 204$ & $119 / 188$ & $3 / 152$ & $2 / 117$ & $3 / 181$ & $0 / 107$ & $4 / 247$ & $117 / 270$ & $67 / 110$ & $69 / 133$ & $17 / 31$ \\
\hline ratio & $98.4 \%$ & $88.2 \%$ & $63.3 \%$ & $2.0 \%$ & $1.7 \%$ & $1.7 \%$ & $0.0 \%$ & $1.6 \%$ & $63.0 \%$ & $60.9 \%$ & $51.8 \%$ & $54.8 \%$ \\
\hline after & $488 / 505$ & $319 / 351$ & $171 / 279$ & $181 / 352$ & $115 / 211$ & $81 / 290$ & $64 / 285$ & $137 / 306$ & $268 / 447$ & $228 / 297$ & $140 / 229$ & $80 / 108$ \\
\hline ratio & $96.6 \%$ & $90.8 \%$ & $61.3 \%$ & $51.4 \%$ & $54.5 \%$ & $27.9 \%$ & $22.5 \%$ & $44.8 \%$ & $59.9 \%$ & $76.8 \%$ & $61.1 \%$ & $74.1 \%$ \\
\hline
\end{tabular}

\begin{tabular}{|c|c|c|c|c|c|c|c|}
\hline \multicolumn{8}{|c|}{ Table S4. Assembly efficiency of logic gates } \\
\hline & \multirow[b]{2}{*}{ inputs } & \multicolumn{3}{|c|}{ data-1 } & \multicolumn{3}{|c|}{ data-2 } \\
\hline & & Not fully-changed & Fully-changed & Ratio & Not fully-changed & Fully-changed & Ratio \\
\hline \multirow{4}{*}{ AND } & {$[0,0]$} & 735 & 5 & $0.68 \%$ & 694 & 6 & $0.86 \%$ \\
\hline & {$[0,1]$} & 681 & 9 & $1.30 \%$ & 816 & 6 & $0.73 \%$ \\
\hline & {$[1,0]$} & 772 & 4 & $0.52 \%$ & 701 & 2 & $0.28 \%$ \\
\hline & {$[1,1]$} & 318 & 1157 & $78.44 \%$ & 370 & 1301 & $77.86 \%$ \\
\hline \multirow{2}{*}{ OR } & {$[0,0]$} & - & - & - & - & - & - \\
\hline & {$[0,1]$} & 354 & 1437 & $80.23 \%$ & 406 & 1610 & $79.86 \%$ \\
\hline
\end{tabular}




\begin{tabular}{|c|c|c|c|c|c|c|c|}
\hline & {$[1,0]$} & 444 & 1007 & $69.40 \%$ & 611 & 1215 & $66.54 \%$ \\
\hline & {$[1,1]$} & $387(773 / 2)$ & 1321 & $77.34 \%$ & $448(895 / 2)$ & 1590 & $76.05 \%$ \\
\hline \multirow{4}{*}{ XOR } & {$[0,0]$} & - & - & - & - & - & - \\
\hline & {$[0,1]$} & 1204 & 2717 & $69.30 \%$ & 1297 & 2556 & $66.34 \%$ \\
\hline & {$[1,0]$} & 858 & 1870 & $68.55 \%$ & 901 & 1910 & $67.95 \%$ \\
\hline & {$[1,1]$} & $676(1352 / 2)$ & 192 & $22.12 \%$ & $1385(2770 / 2)$ & 362 & $20.72 \%$ \\
\hline \multirow{4}{*}{ NAND } & {$[0,0]$} & 833 & 1835 & $68.78 \%$ & 714 & 2061 & $74.27 \%$ \\
\hline & {$[0,1]$} & $661(1321 / 2)$ & 995 & $60.08 \%$ & $754(1508 / 2)$ & 936 & $55.38 \%$ \\
\hline & {$[1,0]$} & $695(1390 / 2)$ & 1274 & $64.70 \%$ & $776(1551 / 2)$ & 1210 & $60.91 \%$ \\
\hline & {$[1,1]$} & $334(1003 / 3)$ & 171 & $33.90 \%$ & $421(1263 / 3)$ & 196 & $31.77 \%$ \\
\hline \multirow{4}{*}{ NOR } & {$[0,0]$} & - & - & - & - & - & - \\
\hline & {$[0,1]$} & $602(1203 / 2)$ & 15 & $2.43 \%$ & $585(1170 / 2)$ & 9 & $1.52 \%$ \\
\hline & {$[1,0]$} & $588(1176 / 2)$ & 6 & $1.01 \%$ & $571(1141 / 2)$ & 4 & $0.70 \%$ \\
\hline & {$[1,1]$} & $419(1257 / 3)$ & 17 & $3.90 \%$ & $394(1182 / 3)$ & 5 & $1.25 \%$ \\
\hline \multirow{4}{*}{ XNOR } & {$[0,0]$} & - & - & - & - & - & - \\
\hline & {$[0,1]$} & $341(681 / 2)$ & 76 & $18.22 \%$ & $362(724 / 2)$ & 58 & $13.81 \%$ \\
\hline & {$[1,0]$} & $419(837 / 2)$ & 55 & $11.60 \%$ & $348(695 / 2)$ & 47 & $11.90 \%$ \\
\hline & {$[1,1]$} & $386(771 / 2)$ & 523 & $57.54 \%$ & & & \\
\hline
\end{tabular}

\begin{tabular}{|c|c|c|c|c|}
\hline \multicolumn{3}{|c|}{ Table S5. Assembly efficiency of Majority function } \\
\hline \multirow{3}{*}{ majority } & inputs & Unchanged & comb-array & $145(11.1 \%)$ \\
\cline { 2 - 5 } & {$[0,0,0]$} & $1302(87.7 \%)$ & $261(25.5 \%)$ & $36(1.2 \%)$ \\
\cline { 2 - 5 } & {$[1,0,0]$} & $583(1165 / 2)(56.9 \%)$ & $364(31.5 \%)$ & $180(17.6 \%)$ \\
\cline { 2 - 5 } & {$[0,1,0]$} & $672(1343 / 2)(58.2 \%)$ & $118(10.3 \%)$ & ladder-array \\
\hline
\end{tabular}




\begin{tabular}{|c|c|c|c|c|}
\hline \multirow{4}{*}[0,0,1]{} & $744(1488 / 2)(59.8 \%)$ & $351(28.3 \%)$ & $147(11.9 \%)$ \\
\cline { 2 - 5 } & {$[1,1,0]$} & $246(737 / 3)(28.5 \%)$ & $204(23.7 \%)$ & $412(47.8 \%)$ \\
\cline { 2 - 5 } & {$[1,0,1]$} & $237(712 / 3)(33.5 \%)$ & $193(27.3 \%)$ & $276(39.2 \%)$ \\
\cline { 2 - 5 } & {$[0,1,1]$} & $287(861 / 3)(29.1 \%)$ & $327(33.2 \%)$ & $371(37.7 \%)$ \\
\cline { 2 - 5 } & {$[1,1,1]$} & $136(272 / 2)(6.1 \%)$ & $605(27 \%)$ & $1497(66.9 \%)$ \\
\hline
\end{tabular}




\section{Sequences ( $\left({ }^{\prime}\right.$ ' to 3')}

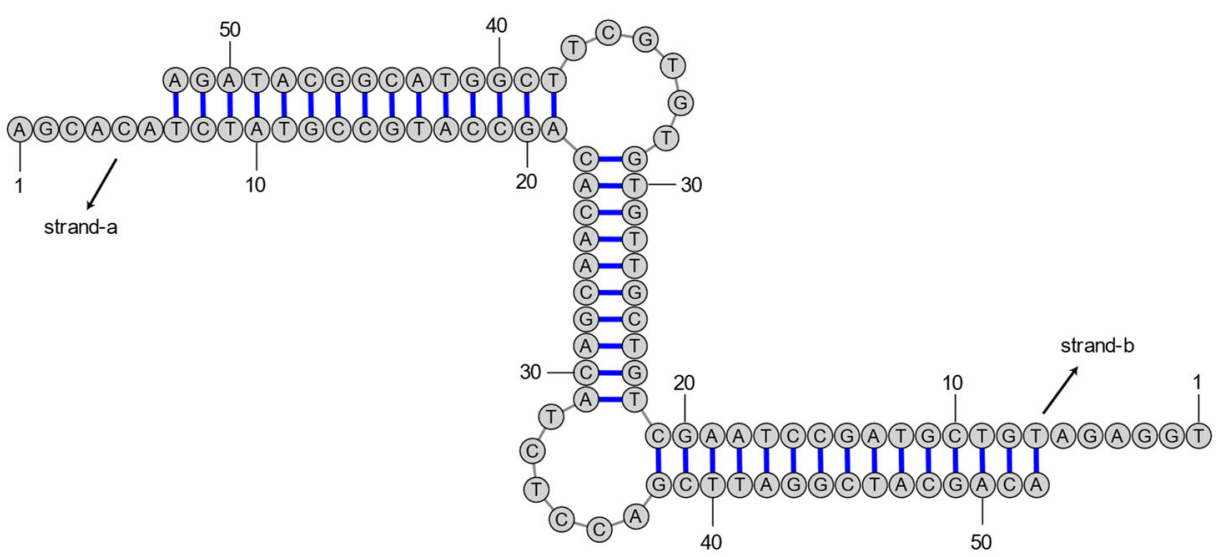

Figure S57. Schematics showing the sequence arrangement of a Z-switch model which is composed of two strands - strand a and strand b. Hereinafter the sequences of all Z-switches in this study are marked in this way.

\begin{tabular}{|c|c|}
\hline \multicolumn{2}{|c|}{ Z-switches with flexible stems in Figure S2. } \\
\hline Flexible 1-a & 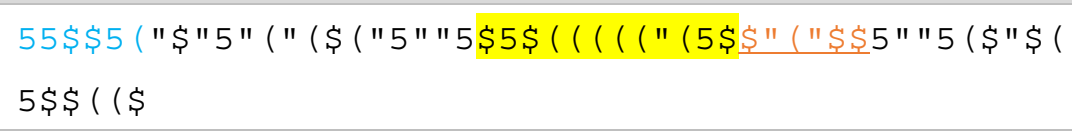 \\
\hline Flexible 1-b & 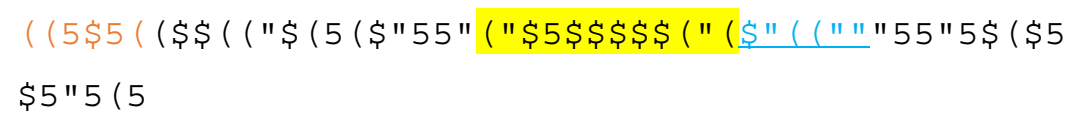 \\
\hline \multicolumn{2}{|c|}{ Z-switches with 1 site blocked } \\
\hline TFO & $5 \$ \$ 5 \$ 55 \$ 5 \$ 5 \$$ \\
\hline S1-a & 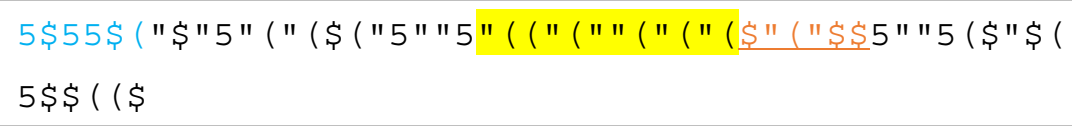 \\
\hline S1-b & 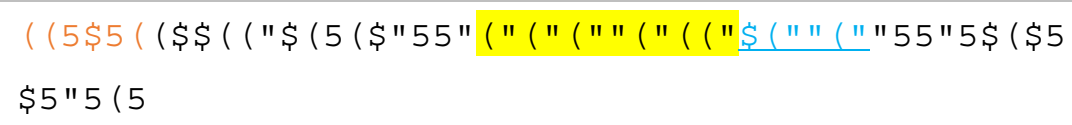 \\
\hline S2-a & 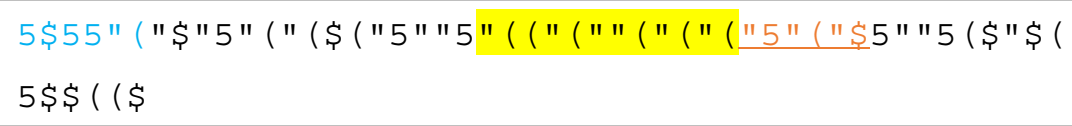 \\
\hline S2-b & 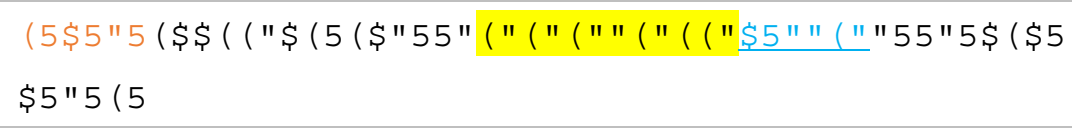 \\
\hline S3-a & 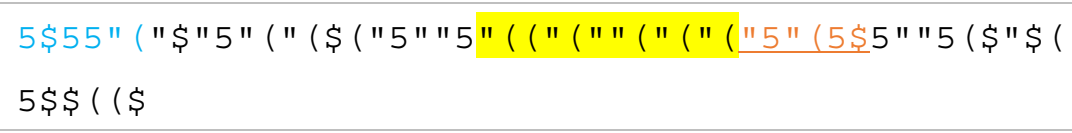 \\
\hline S3-b & 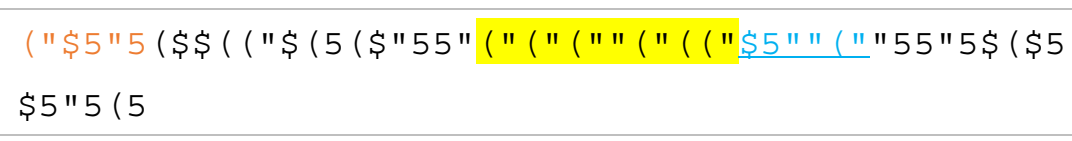 \\
\hline S4-a & 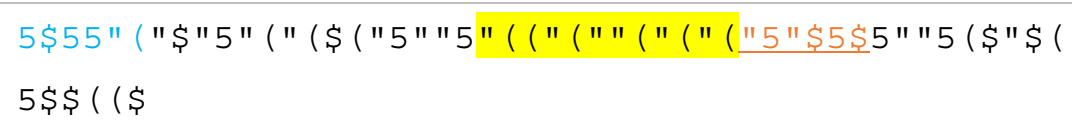 \\
\hline
\end{tabular}




\begin{tabular}{|c|c|}
\hline S4-b & 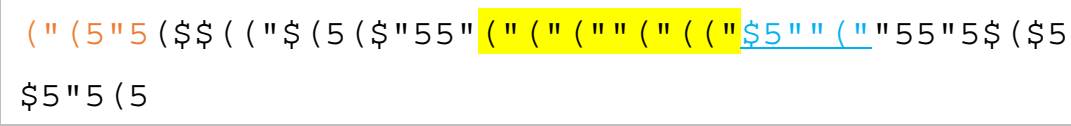 \\
\hline S5-a & 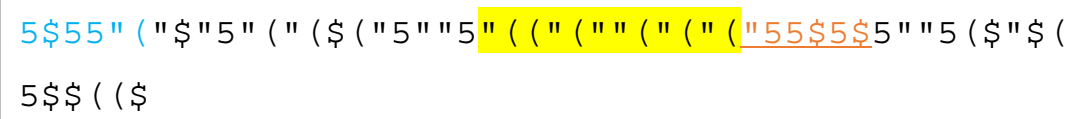 \\
\hline S5-b & 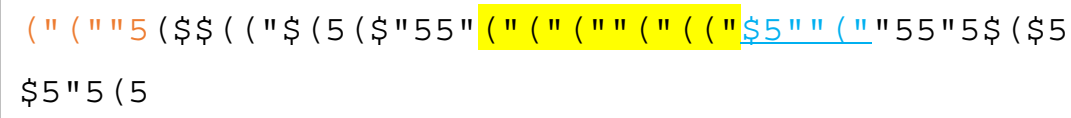 \\
\hline S6-a & 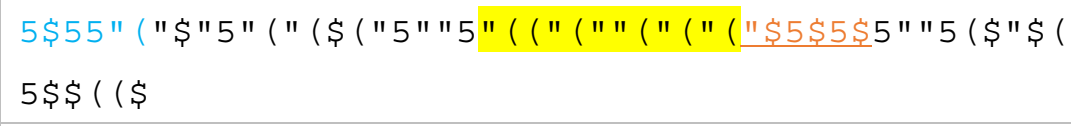 \\
\hline S6-b & 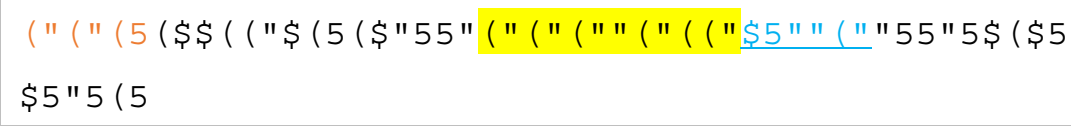 \\
\hline S7-a & 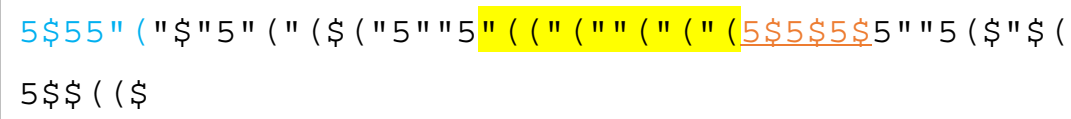 \\
\hline S7-b & 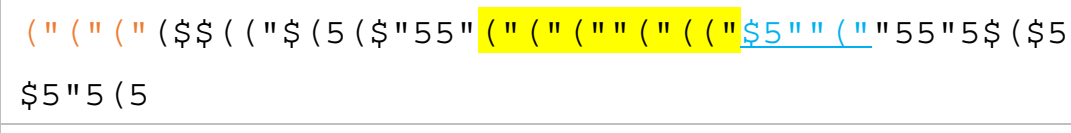 \\
\hline S8-a & 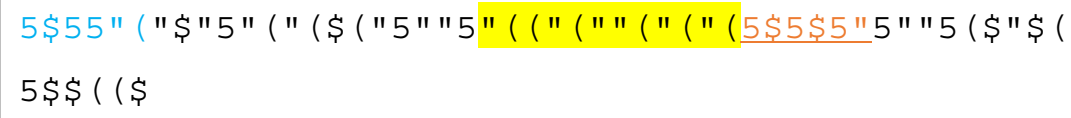 \\
\hline S8-b & 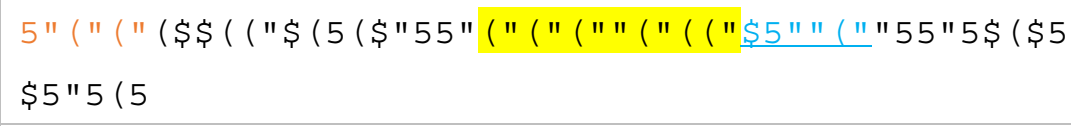 \\
\hline s9-a & 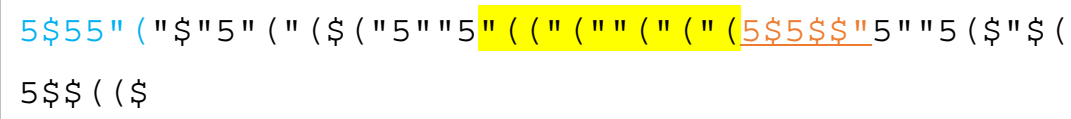 \\
\hline s9-b & 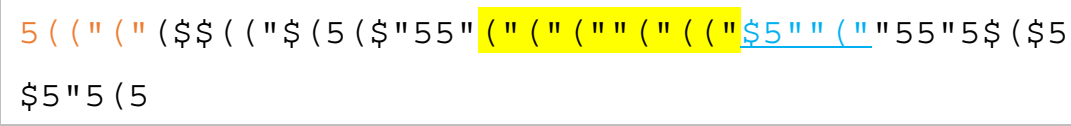 \\
\hline S10-a & 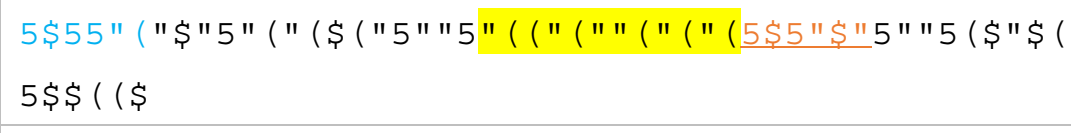 \\
\hline s10-b & 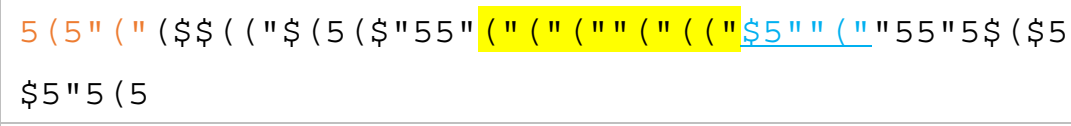 \\
\hline s11-a & 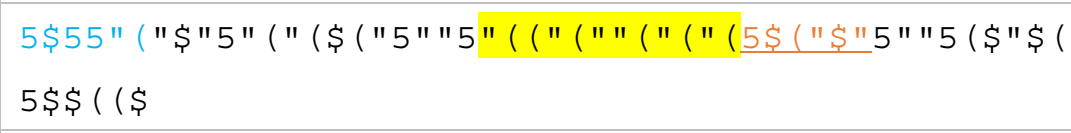 \\
\hline S11-b & 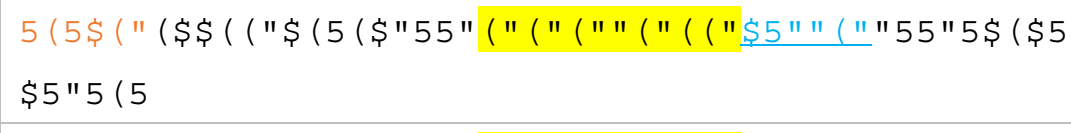 \\
\hline S12-a & 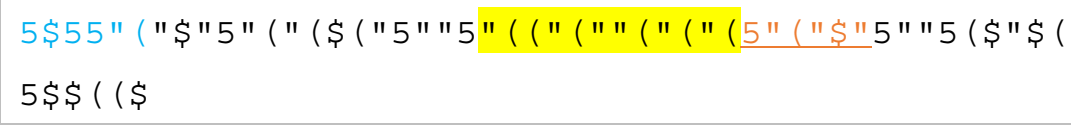 \\
\hline S12-b & 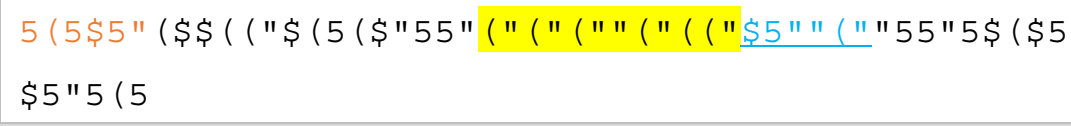 \\
\hline \multicolumn{2}{|c|}{ Z-switches with 1 site blocked } \\
\hline TFO & $5 \$ \$ 5 \$ 55 \$ 5 \$ 5 \$$ \\
\hline D1-a & 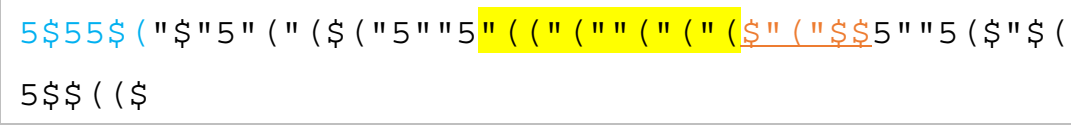 \\
\hline
\end{tabular}




\begin{tabular}{|c|c|}
\hline D1-b & 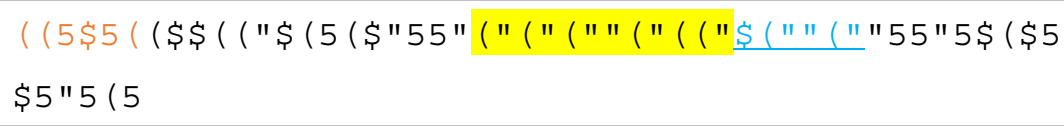 \\
\hline D2-a & 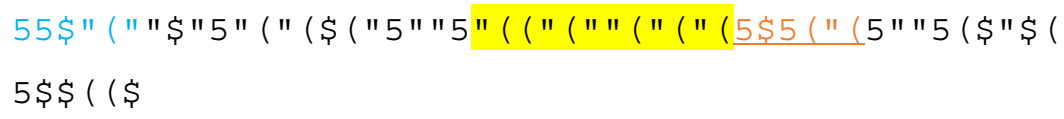 \\
\hline D2-b & 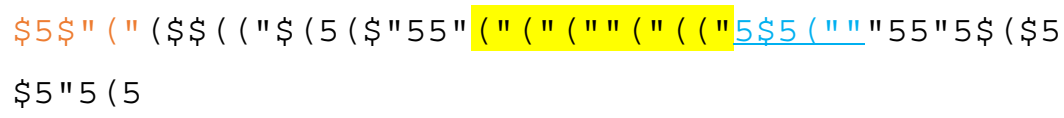 \\
\hline D3-a & 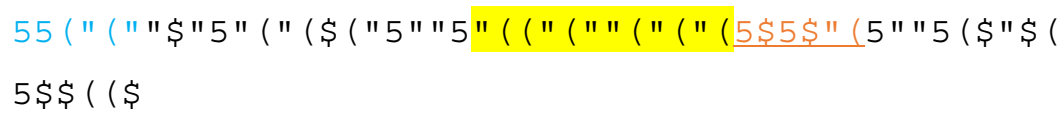 \\
\hline D3-b & 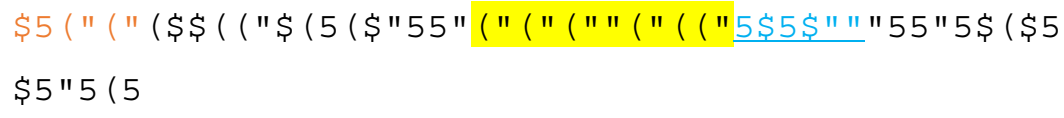 \\
\hline D4-a & 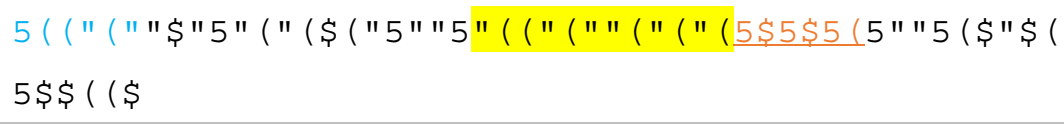 \\
\hline D4-b & 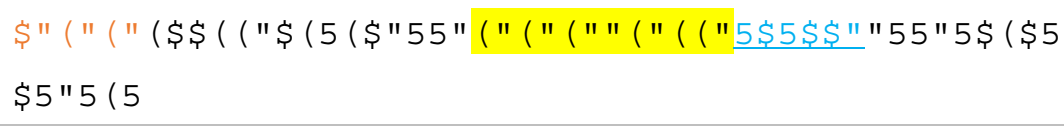 \\
\hline D5-a & 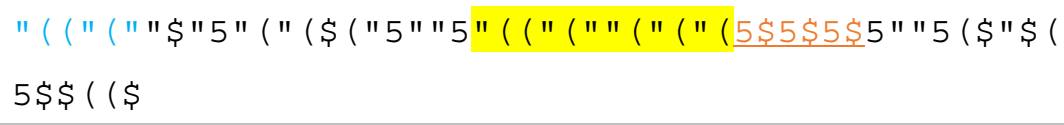 \\
\hline D5-b & 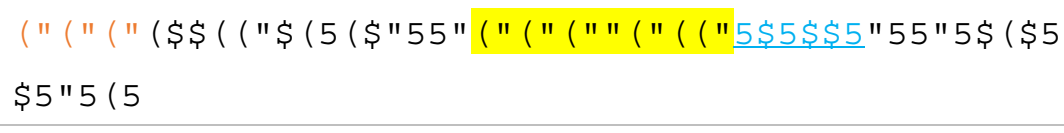 \\
\hline D6-a & 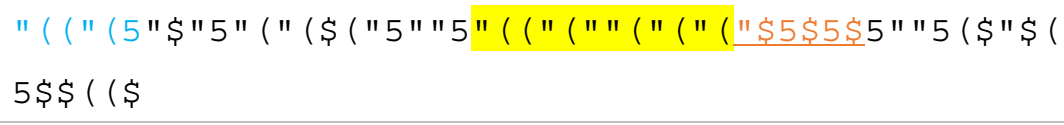 \\
\hline D6-b & 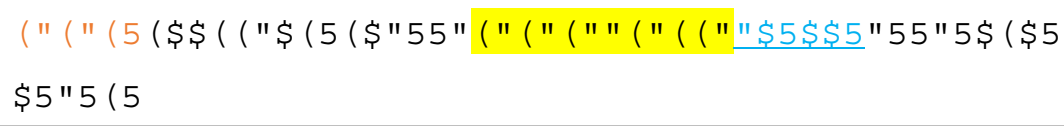 \\
\hline D7-a & 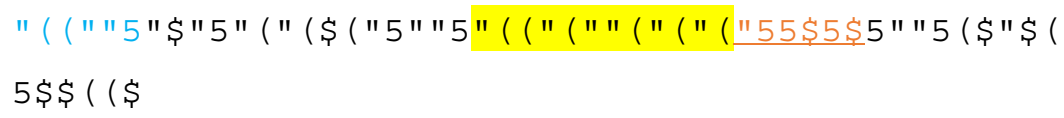 \\
\hline D7-b & 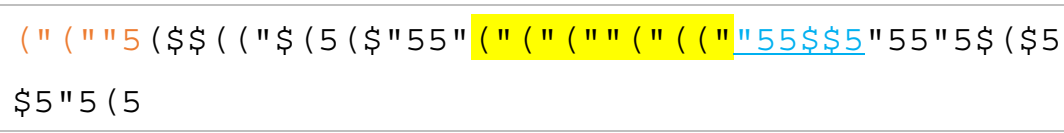 \\
\hline D8-a & 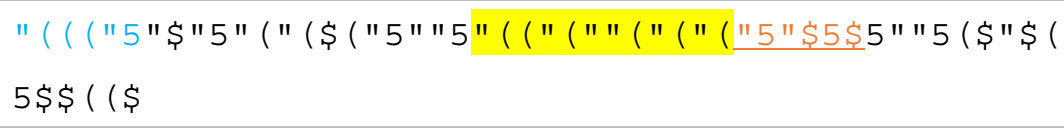 \\
\hline D8-b & 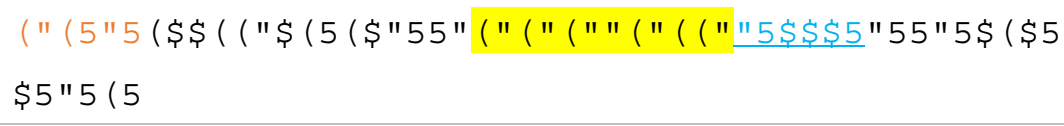 \\
\hline D9-a & 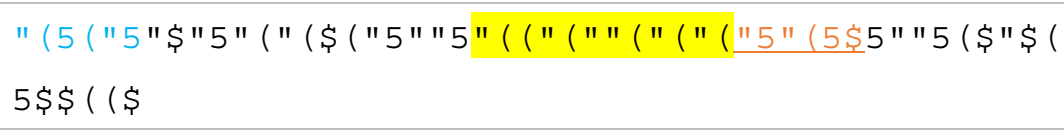 \\
\hline D9-b & 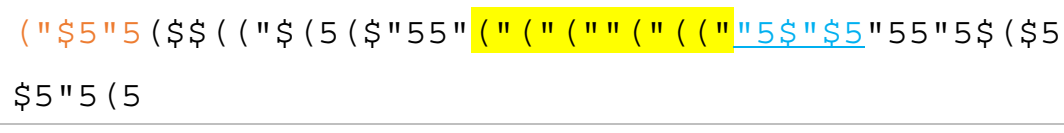 \\
\hline D10-a & 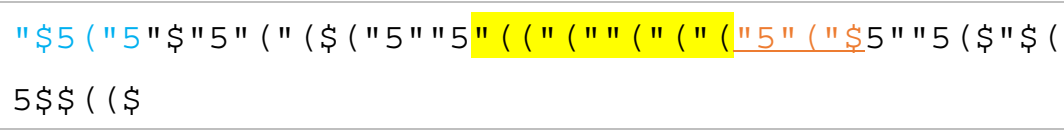 \\
\hline D10-b & 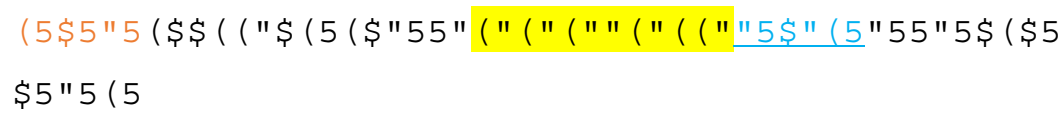 \\
\hline
\end{tabular}




\begin{tabular}{|c|c|}
\hline D11-a & 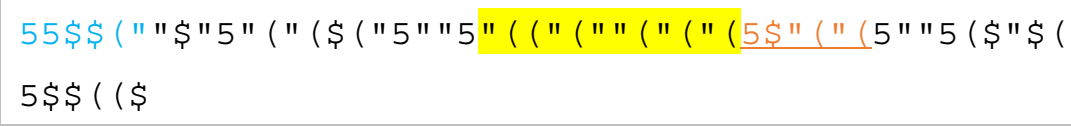 \\
\hline D11-b & 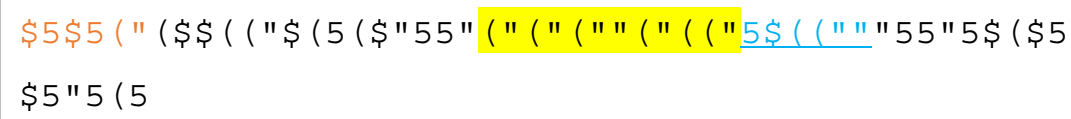 \\
\hline D12-a & 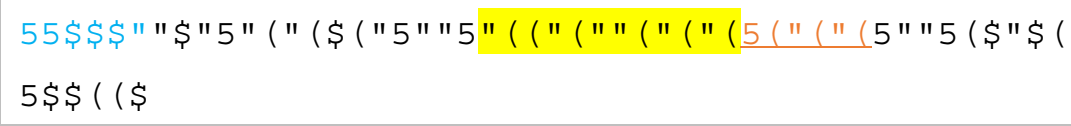 \\
\hline D12-b & 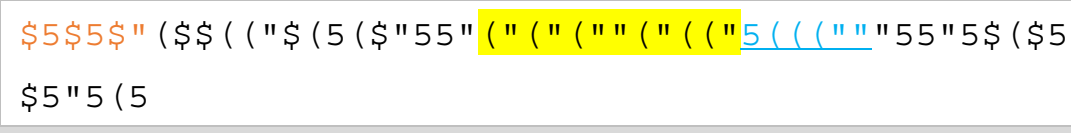 \\
\hline \multicolumn{2}{|c|}{ control group of D3 and D9 } \\
\hline TFO & $5 \$ \$ 5 \$ 55 \$ 5 \$ 5 \$$ \\
\hline D3(V1)-a & 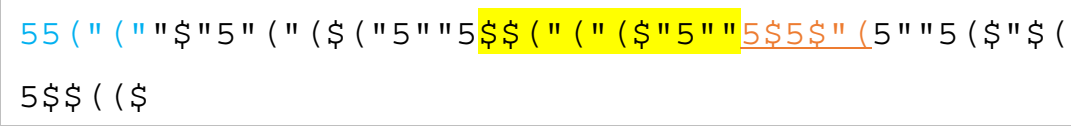 \\
\hline $\mathrm{D} 3(\mathrm{~V} 1)-\mathrm{b}$ & 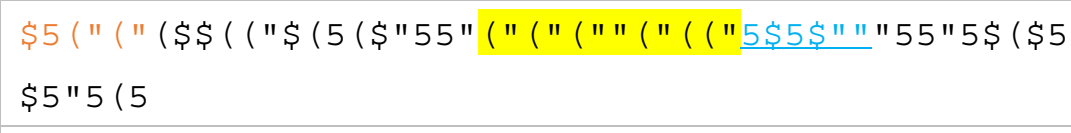 \\
\hline D3(V2)-a & 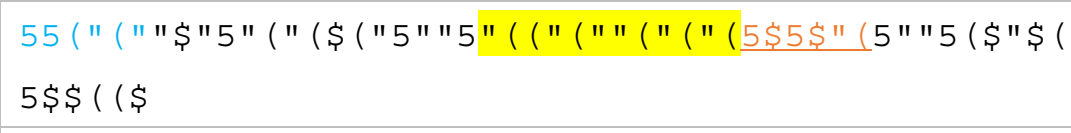 \\
\hline D3(V2)-b & 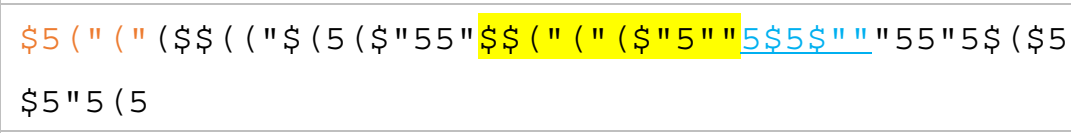 \\
\hline D9(V1)-a & 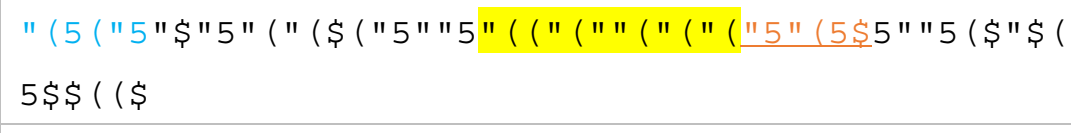 \\
\hline $\mathrm{D} 9(\mathrm{~V} 1)-\mathrm{b}$ & 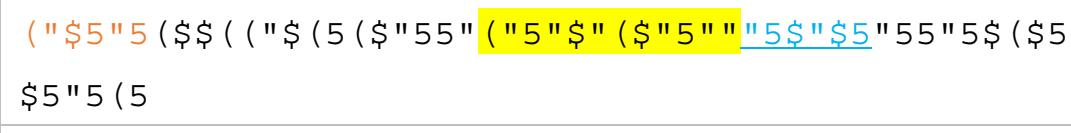 \\
\hline D9(V2)-a & 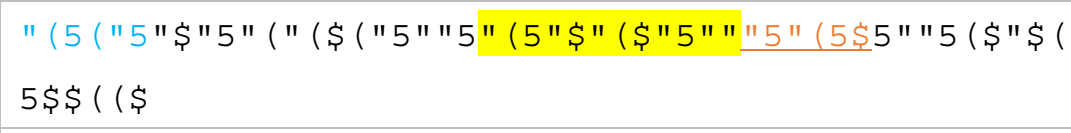 \\
\hline $\mathrm{D} 9(\mathrm{~V} 2)-\mathrm{b}$ & 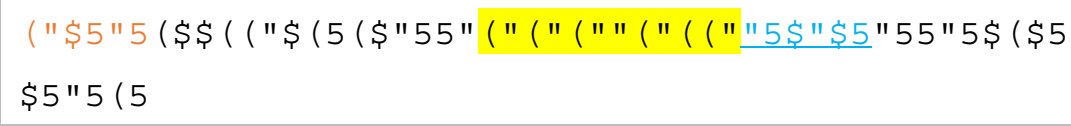 \\
\hline \multicolumn{2}{|c|}{ Z-switch that can assemble into 2D Network } \\
\hline TFO $(x-$ form $)$ & $5 \$ \$ 5 \$ 55 \$ 5 \$ 5 \$$ \\
\hline TFO (y - form) & $\$ 5 \$ 5 \$ 55 \$ 5 \$ \$ 5$ \\
\hline Network-a & 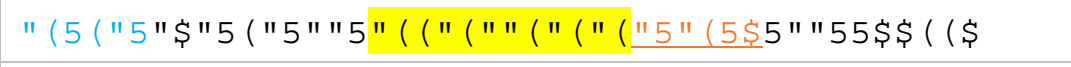 \\
\hline Network-b & 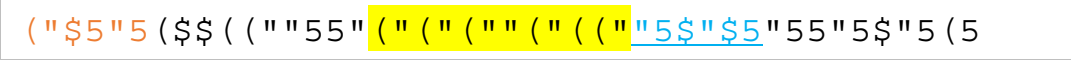 \\
\hline \multicolumn{2}{|c|}{ Z-switch that can assemble into 2D Wheel } \\
\hline TFO (x - form) & \$5\$5\$555\$555\$55\$\$\$5\$5\$\$5 \\
\hline TFO (y - form) & $5 \$ \$ 5 \$ 5 \$ \$ 55 \$ 555 \$ 555 \$ 5 \$ 5 \$$ \\
\hline Network-a & 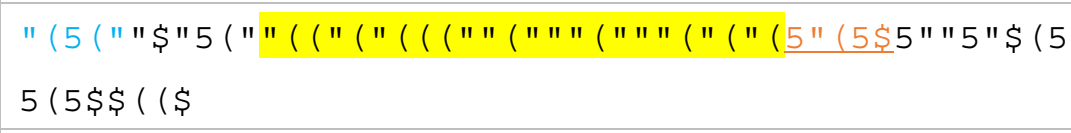 \\
\hline Network-b & 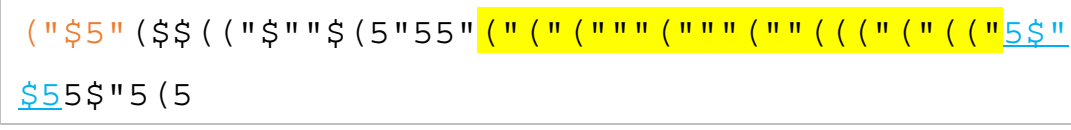 \\
\hline
\end{tabular}


Z-switch that can assemble into 1D Zipper

\begin{tabular}{|c|c|}
\hline TFO (x - form) & $5 \$ \$ 5 \$ 55 \$ 5 \$ 5 \$$ \\
\hline TFO (y - form) & $\$ 5 \$ 5 \$ 55 \$ 5 \$ \$ 5$ \\
\hline Network-a & 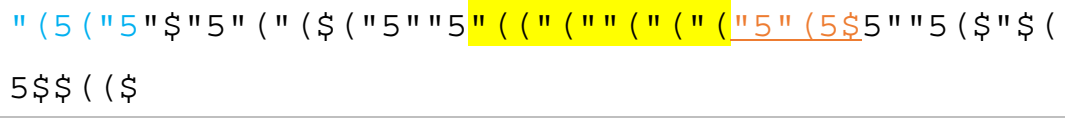 \\
\hline Network-b & 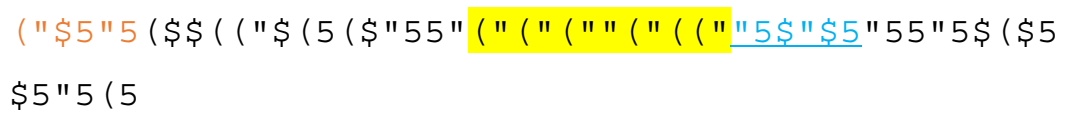 \\
\hline
\end{tabular}

Z-switch that can assemble into 1D Ladder ( $y$ - form)

\begin{tabular}{|c|c|}
\hline TFO (y - form) & $\$ 5 \$ 5 \$ 55 \$ 5 \$ \$ 5$ \\
\hline Network-a & 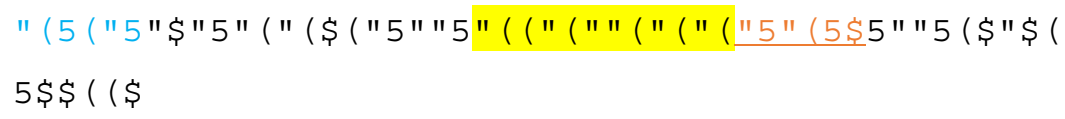 \\
\hline Network-b & 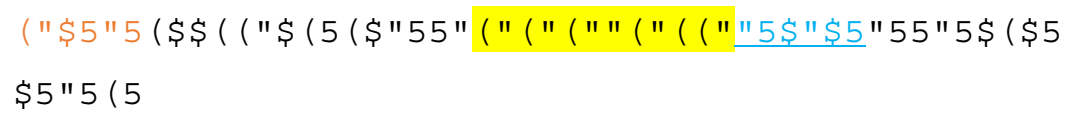 \\
\hline
\end{tabular}

\section{Z-switch for co-regulation}

\begin{tabular}{|c|c|}
\hline TFO M1 & $55 \$ 55 \$ \$ 5 \$ 55 \$$ \\
\hline TFO M2 & $5 \$ \$ 5 \$ 55 \$ 5 \$ 5 \$$ \\
\hline Co Z-switch-a & 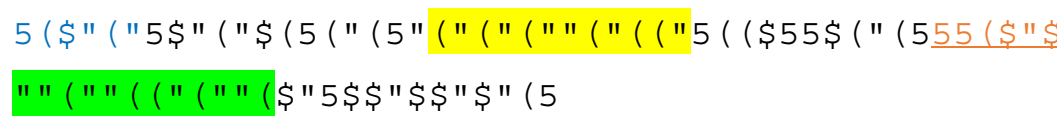 \\
\hline Co Z-switch-b & 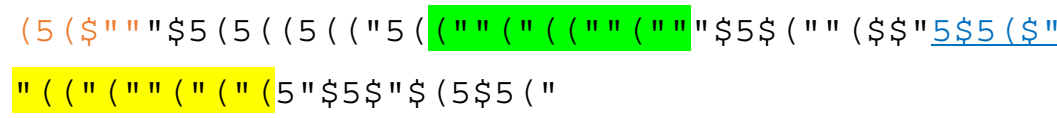 \\
\hline
\end{tabular}

\section{Z-switch for logic gates}

\begin{tabular}{|c|c|}
\hline AND-a & 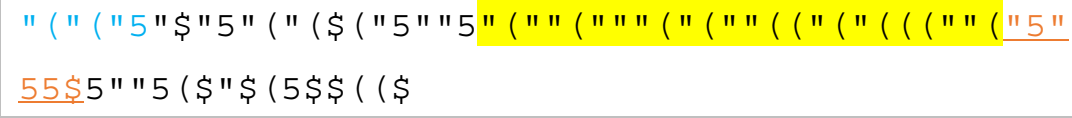 \\
\hline AND-b & 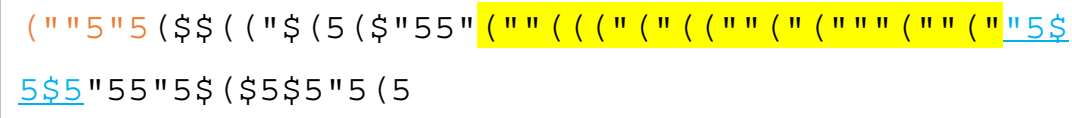 \\
\hline AND-in1 & $5 \$ 5 \$ 555 \$ 55 \$ 5$ \\
\hline AND-in2 & $\$ 55 \$ \$ 5 \$ 5 \$ \$ 5$ \\
\hline OR-a & 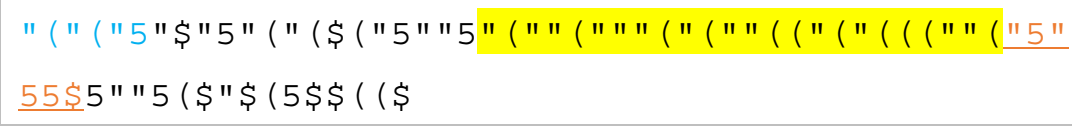 \\
\hline OR-b & 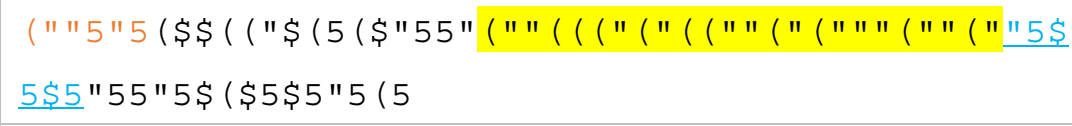 \\
\hline OR-in1 & $\$ 55 \$ \$ \$ 5 \$ 5 \$ 55 \$ 5 \$ 555 \$ 55 \$ 5$ \\
\hline OR-in2 & $5 \$ 55 \$ 555 \$ 5 \$ 55 \$ \$ 5 \$ 5 \$ \$ \$ 55$ \\
\hline XOR-a & 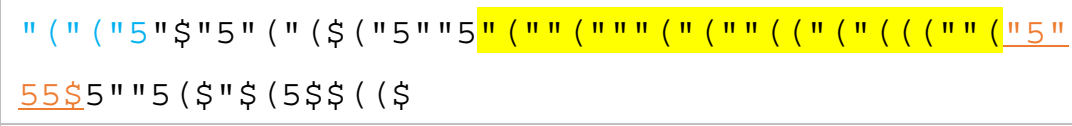 \\
\hline XOR-b & 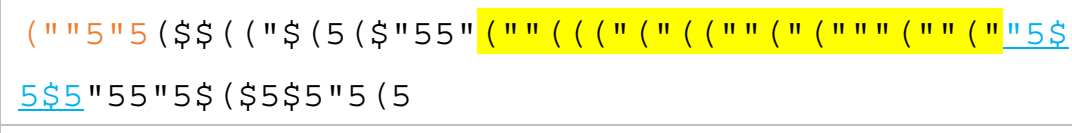 \\
\hline XOR-in 1 & 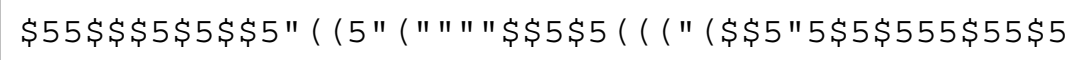 \\
\hline
\end{tabular}




\begin{tabular}{|c|c|}
\hline XOR-in2 & 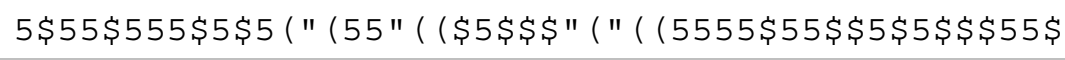 \\
\hline NAND-a & 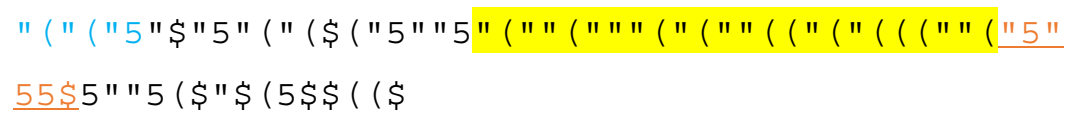 \\
\hline NAND-b & 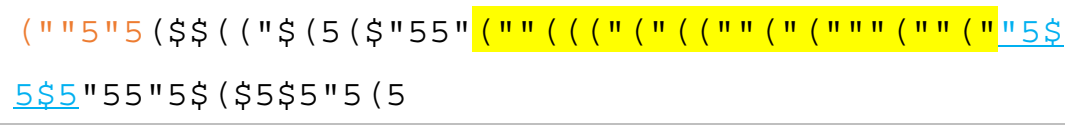 \\
\hline NAND-TFO & 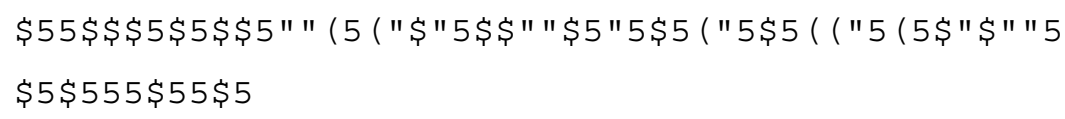 \\
\hline NAND-in $1-1$ & 5 " 5 " ( 55 ( ( " 5 (5\$"\$55") ( " ( " ( ( ( " ") \\
\hline NAND-in $1-2$ & 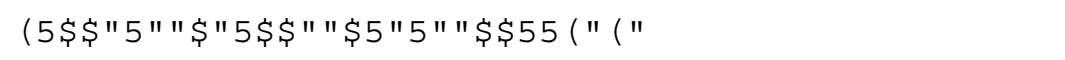 \\
\hline NAND-in2-1 & 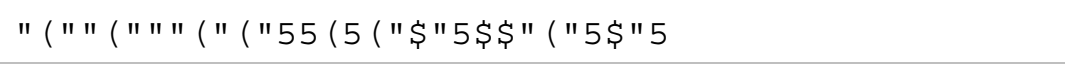 \\
\hline NAND-in2-2 & 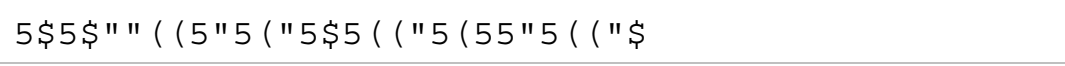 \\
\hline NOR-a & 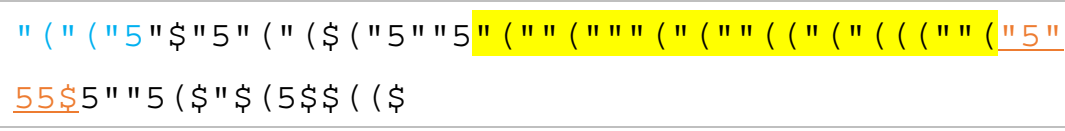 \\
\hline NOR-b & 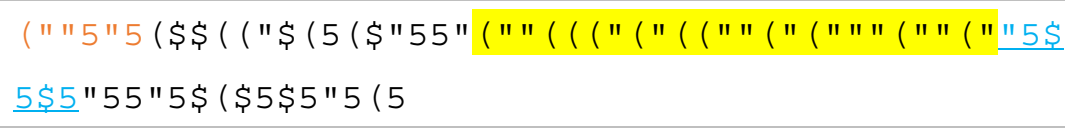 \\
\hline NOR-TFO & 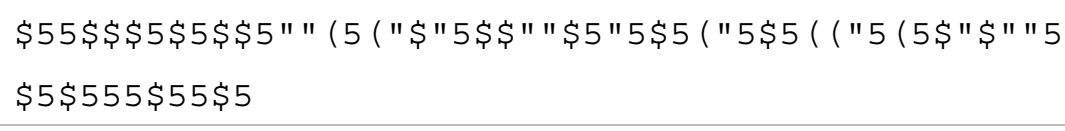 \\
\hline NOR-in1 & 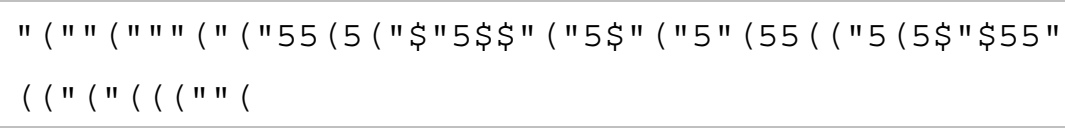 \\
\hline NOR-in2 & 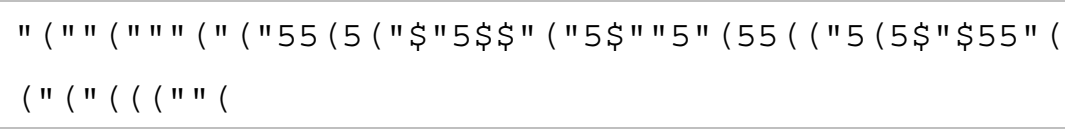 \\
\hline XNOR-a & 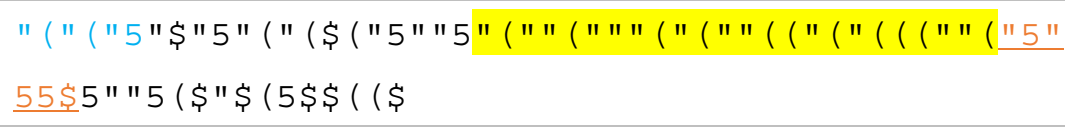 \\
\hline XNOR-b & 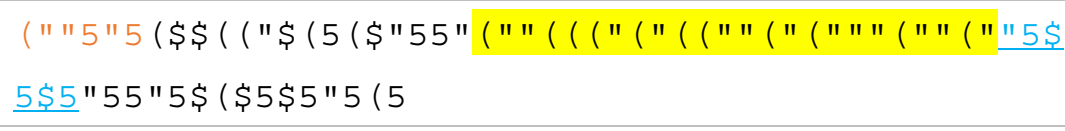 \\
\hline XNOR-TFO & 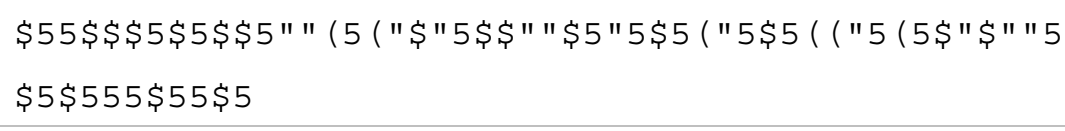 \\
\hline XNOR-in1 & 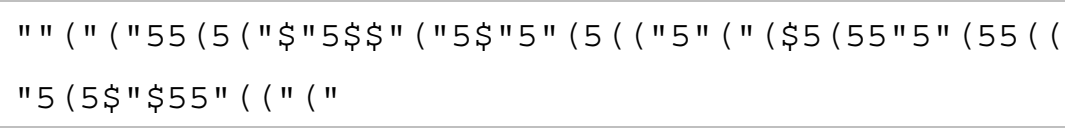 \\
\hline XNOR-in2 & 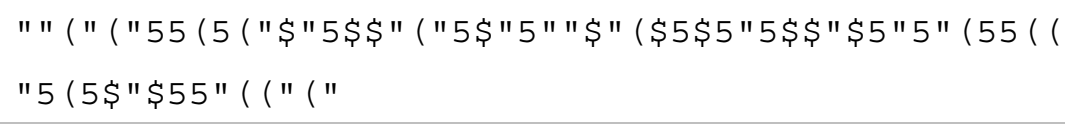 \\
\hline MAJORITY-Z1-a & 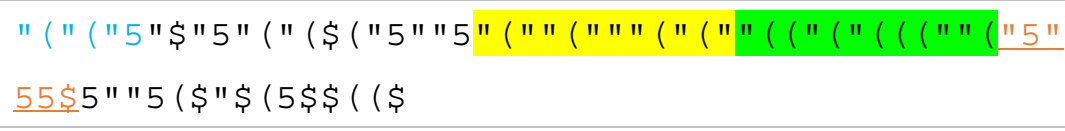 \\
\hline MAJORITY-Z1-b & 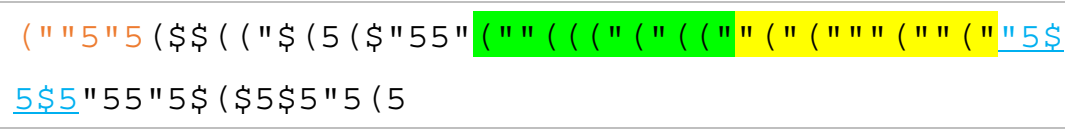 \\
\hline MAJORITY-Z2-a & 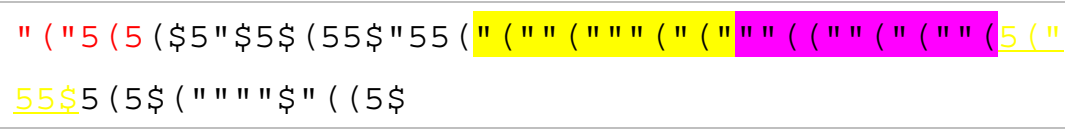 \\
\hline MAJORITY-Z2-b & 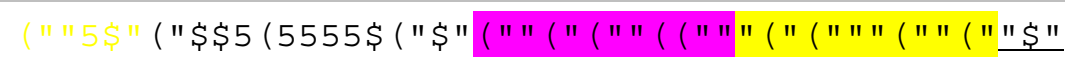 \\
\hline
\end{tabular}




\begin{tabular}{|c|c|}
\hline & $\underline{5 \$ 5} \$$ " " 5 ( " "\$ ( " ( 5 " (\$ \\
\hline \multirow{2}{*}{ MAJORITY-Z3-a } & 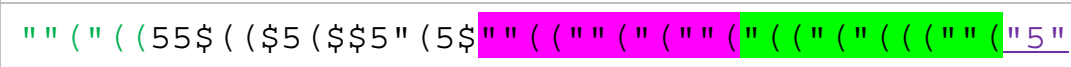 \\
\hline & 55\$\$ ( "\$ ( (" (5) ( 5) " ( \\
\hline \multirow{2}{*}{ MAJORITY-Z3-b } & 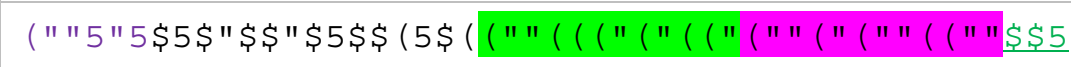 \\
\hline & \$55 ( "\$5"（（\$"（\$\$（" " \\
\hline $\ln -1$ & $5 \$ 5 \$ 555 \$ 55 \$ 5$ \\
\hline $\ln -2$ & \$5 $5 \$ \$ 5 \$ 5 \$ \$ 5$ \\
\hline $\ln -3$ & $55 \$ \$ 55 \$ 5 \$ 55 \$$ \\
\hline
\end{tabular}

Table S6. Sequences used in this study. All sequences are written from 5 ' to 3 '. In each sequence, triplex-forming regions are highlighted. Active sites are colored and underlined and the corresponding 5 ' overhangs are marked in the same color but not underlined. 